University of Tennessee Health Science Center UTHSC Digital Commons

\title{
Determination of the Functional Relationship Between Lumbar Lordosis and Pelvic Tilt
}

\author{
Casey Tyler Hebert \\ University of Tennessee Health Science Center
}

Follow this and additional works at: https://dc.uthsc.edu/dissertations

Part of the Surgical Procedures, Operative Commons

\section{Recommended Citation}

Hebert, Casey Tyler, "Determination of the Functional Relationship Between Lumbar Lordosis and Pelvic Tilt" (2014). Theses and Dissertations (ETD). Paper 104. http://dx.doi.org/10.21007/etd.cghs.2014.0131.

This Thesis is brought to you for free and open access by the College of Graduate Health Sciences at UTHSC Digital Commons. It has been accepted for inclusion in Theses and Dissertations (ETD) by an authorized administrator of UTHSC Digital Commons. For more information, please contact jwelch30@uthsc.edu. 


\title{
Determination of the Functional Relationship Between Lumbar Lordosis and Pelvic Tilt
}

\begin{abstract}
Americans are undergoing spinal fusion surgery (SFS) at an ever increasing rate; in 2008 over 400,000 Americans underwent SFS with a national cost of approximately $\$ 33.9$ billion. During SFS it is difficult for the surgeon to properly align the spine's s-shape, as viewed from the patient's side. Abnormal alignment of the spine then alters the position of the pelvis and hip joints, which may impact the function and hip contact patterns. Several studies have shown that patients with spinal pathology, such as arthritis, often have a coexisting hip pathology or subsequently develop hip pathology, and it is estimated that $18 \%$ of individuals undergoing total hip arthroplasty (THA) have concurrently developed a lumbar spine disorder. If the THA patient then undergoes SFS, any abnormal alignment of the spine can then impact the function and survivorship of the THA. The goal of this study was to examine the influence of altered sagittal lumbar lordosis on sagittal pelvis kinematics during activities of daily living including gait, sit-to-stand, and standto-sit. We hypothesized that a subject would compensate an altered lumbar lordosis by manipulating their pelvic tilt and torso alignment to maintain a normal plumbline. To investigate this hypothesis 10 healthy subjects ( 6 female, 4 male), aged 18-35 years, with no back, spine, or lower extremity injuries or surgeries were evaluated during static stance, gait, sit-to-stand, and stand-to-sit. Subjects performed activities in a 3-D motion analysis lab, with and without the use of a hyper-tensioned clavicle strap. An EOS bi-planar x-ray system was used to validate marker placement, as well as spinal and pelvic changes induced by the hypertension clavicle strap. Each subject also underwent a standard physical therapy exam to determine any functional limitations or abnormalities. Subjects were then evaluated in a paired fashion. Changes in pelvic tilt and hip flexion were correlated to changes in lumbar lordosis, plumbline, and trunk-pelvic angle. When different groups were present, the statistical coincidence of each linear regression was tested. For each condition a Wilcoxon signed-rank test was used to determine if each of the aforementioned parameters significantly changed from normal. This study found that decreasing lumbar lordosis by (mean \pm SD) $4 \pm 2$ deg during gait did not significantly alter pelvic tilt. However, subjects with clinically tight hamstrings responded significantly different to a hyper-tensioned clavicle strap. Similarly, -5 to 9 deg change in lumbar lordosis did not correlate with changes in pelvic tilt during sit-to-stand or stand-to-sit activities. Changes in plumbline were found to be the best predictor for changes in pelvic tilt at peak hip flexion during stand-to-sit and sit-to-stand activities, exhibiting a nearly 1:1 relationship.
\end{abstract}

\section{Document Type}

Thesis

\section{Degree Name}

Master of Science (MS)

\section{Program}

Biomedical Engineering and Imaging

\section{Research Advisor}

William M. Mihalko, M.D., Ph.D.

\section{Keywords}

Fixed Sagittal Imbalance, Flatback, Kinematics, Lumbar, Pelvis, Validation 


\section{Subject Categories}

Analytical, Diagnostic and Therapeutic Techniques and Equipment | Medicine and Health Sciences |

Surgical Procedures, Operative 


\title{
DETERMINATION OF THE \\ FUNCTIONAL RELATIONSHIP BETWEEN \\ LUMBAR LORDOSIS AND PELVIC TILT
}

\author{
A Thesis \\ Presented for \\ The Graduate Studies Council \\ The University of Tennessee \\ Health Science Center
}

\begin{abstract}
In Partial Fulfillment
Of the Requirements for the Degree

Master of Science in Biomedical Engineering

In the Joint Graduate Program in Biomedical Engineering and Imagining

From The University of Tennessee

and

The University of Memphis
\end{abstract}

By

Casey Tyler Hebert

August 2014 
Copyright (C) 2014 by Casey Tyler Hebert. All rights reserved. 


\section{DEDICATION}

For my grandparents Donald D. Ewbank, Virginia M. Ewbank, and Ernestine V. Hebert: While I did not have much time with you in this world, your kindness and legacy have driven me to be the man I am today.

For my family Verne J. Hebert, Kimberly C. Hebert, and Kassandra N. Hebert:

Without your support and drive I would have never made it this far. Thank you teaching me how to think, work, and succeed.

I dedicate this thesis to you all, because without you I would have never pushed myself to be better. This work was only able to be done by me because of you. Thank you all. 


\section{ACKNOWLEDGEMENTS}

I would like to thank my advisor, Dr. William M. Mihalko for giving me this great opportunity and for all of his guidance. I would also like to thank my committee members, Dr. Audrey R. Zucker-Levin, Dr. John L. Williams, and Dr. Denis J. DiAngelo for all of their patience and guidance. I would like to give special thanks to Dr. ZuckerLevin for her tireless efforts helping me determine the appropriate lordosis altering device for this study, as well as the countless hours she has spent enhancing my grant writing abilities.

I would like to acknowledge the Memphis Research Consortium for their generous funding.

I would also like to thank Rachel Sidle and everyone at Spears Prosthetics \& Orthotics/ Rehab Services for allowing me to use their facility, as well as their guidance and expertise. I would also like to thank Jodie Kutscher and Quiana Moore at Le Bonheur Children's Hospital for all of their help and patience collecting and processing X-rays. I would also like to thank Dr. Byron Stephens for assisting with measurement validations.

Finally, I would like to acknowledge everyone who has made my graduate experience so enjoyable: Ms. Becky Adams, Erik L. Woodard, Andrew C. Herndon, Hunter J. Smith, and Katherine P. Jones. 


\begin{abstract}
Americans are undergoing spinal fusion surgery (SFS) at an ever increasing rate; in 2008 over 400,000 Americans underwent SFS with a national cost of approximately $\$ 33.9$ billion. During SFS it is difficult for the surgeon to properly align the spine's sshape, as viewed from the patient's side. Abnormal alignment of the spine then alters the position of the pelvis and hip joints, which may impact the function and hip contact patterns. Several studies have shown that patients with spinal pathology, such as arthritis, often have a coexisting hip pathology or subsequently develop hip pathology, and it is estimated that $18 \%$ of individuals undergoing total hip arthroplasty (THA) have concurrently developed a lumbar spine disorder. If the THA patient then undergoes SFS, any abnormal alignment of the spine can then impact the function and survivorship of the THA.

The goal of this study was to examine the influence of altered sagittal lumbar lordosis on sagittal pelvis kinematics during activities of daily living including gait, sitto-stand, and stand-to-sit. We hypothesized that a subject would compensate an altered lumbar lordosis by manipulating their pelvic tilt and torso alignment to maintain a normal plumbline.

To investigate this hypothesis 10 healthy subjects ( 6 female, 4 male), aged 18-35 years, with no back, spine, or lower extremity injuries or surgeries were evaluated during static stance, gait, sit-to-stand, and stand-to-sit. Subjects performed activities in a 3-D motion analysis lab, with and without the use of a hyper-tensioned clavicle strap. An EOS bi-planar x-ray system was used to validate marker placement, as well as spinal and pelvic changes induced by the hypertension clavicle strap. Each subject also underwent a standard physical therapy exam to determine any functional limitations or abnormalities. Subjects were then evaluated in a paired fashion. Changes in pelvic tilt and hip flexion were correlated to changes in lumbar lordosis, plumbline, and trunk-pelvic angle. When different groups were present, the statistical coincidence of each linear regression was tested. For each condition a Wilcoxon signed-rank test was used to determine if each of the aforementioned parameters significantly changed from normal.

This study found that decreasing lumbar lordosis by (mean \pm SD) $4 \pm 2$ deg during gait did not significantly alter pelvic tilt. However, subjects with clinically tight hamstrings responded significantly different to a hyper-tensioned clavicle strap. Similarly, -5 to 9 deg change in lumbar lordosis did not correlate with changes in pelvic tilt during sit-to-stand or stand-to-sit activities. Changes in plumbline were found to be the best predictor for changes in pelvic tilt at peak hip flexion during stand-to-sit and sitto-stand activities, exhibiting a nearly 1:1 relationship.
\end{abstract}




\section{TABLE OF CONTENTS}

CHAPTER 1. INTRODUCTION ................................................................................

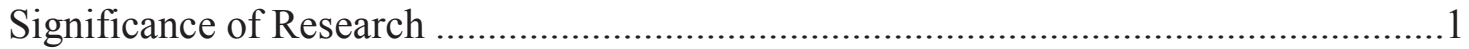

Thesis Aim and Outline ....................................................................................4

Overview of Pelvic and Spinal Anatomy ...............................................................4

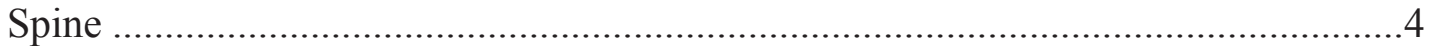

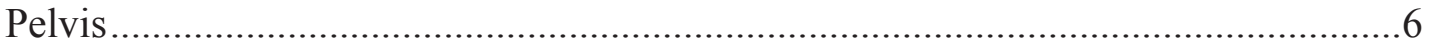

Overview of Pelvic, Spine, and Study Measurements .............................................6

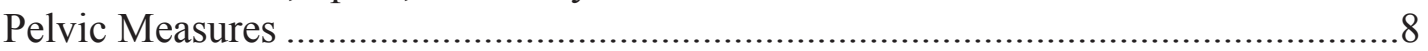

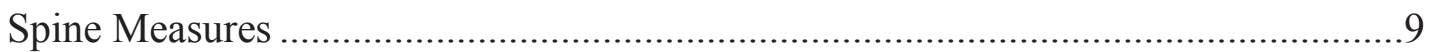

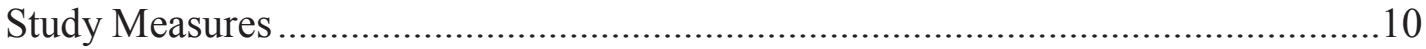

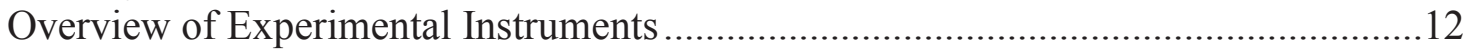

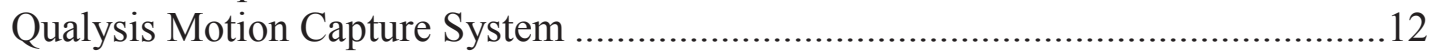

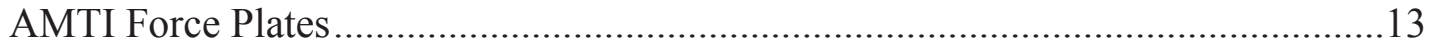

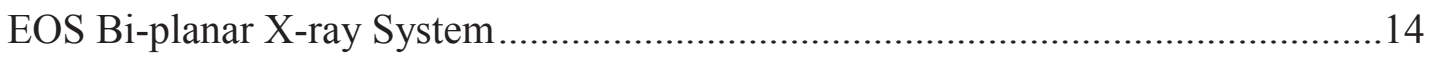

\section{CHAPTER 2. VALIDATION OF MOTION ANALYSIS SPINAL AND PELVIC} MEASURES USING AN EOS X-RAY SYSTEM...............................................18

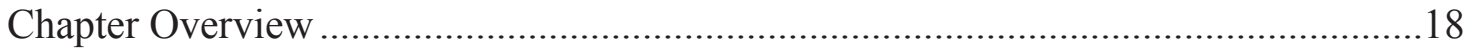

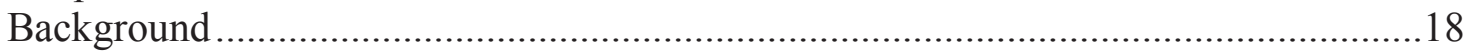

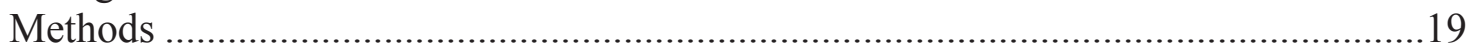

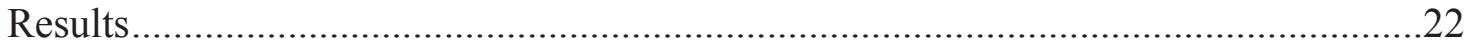

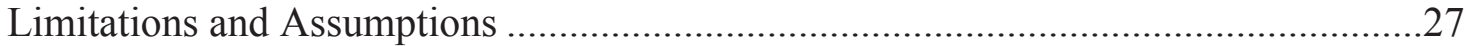

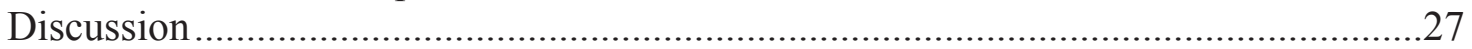

CHAPTER 3. EOS BI-PLANAR X-RAY VALIDATES HYPER-TENSIONED

CLAVICLE STRAP ALTERS LUMBAR LORDOSIS IN STANDING ..................29

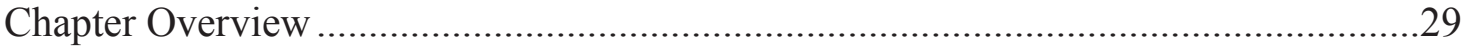

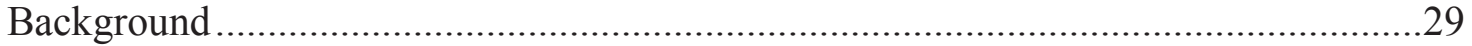

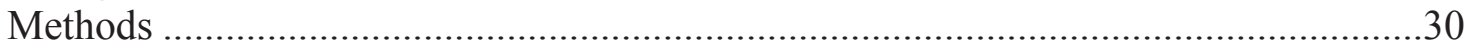

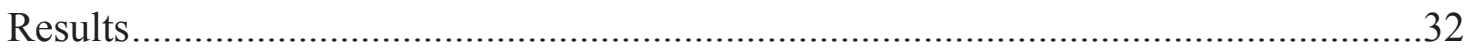

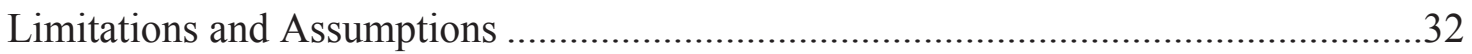

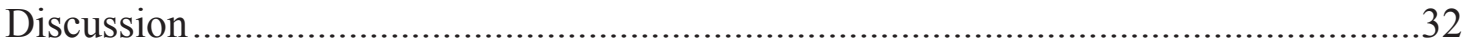

\section{CHAPTER 4. SPINO-PELVIC KINEMATICS OF GAIT UNDER DIFFERENT}

SAGITTAL ALIGNMENTS...........................................................................................35

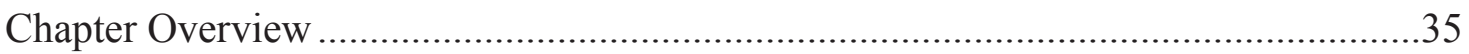

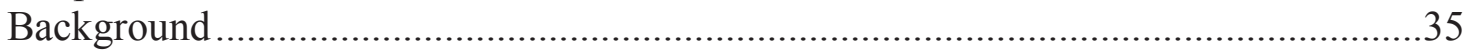

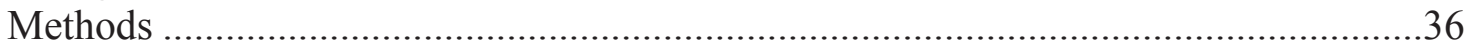

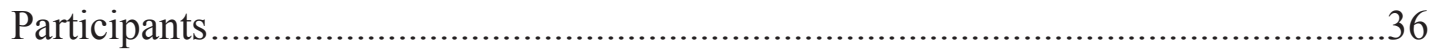

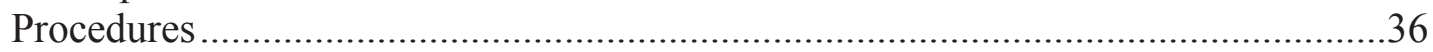

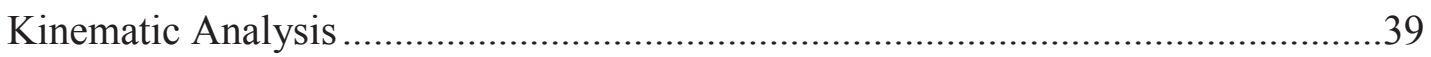

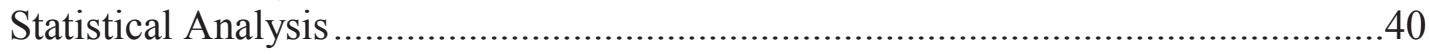




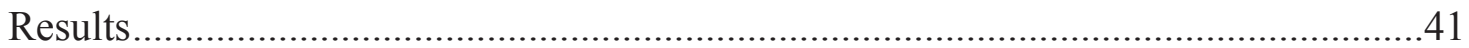

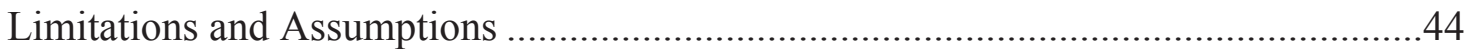

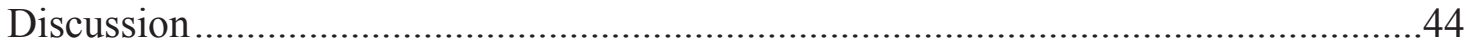

\section{CHAPTER 5. SPINO-PELVIC KINEMATICS OF SIT-TO-STAND AND STAND-TO-SIT ACTIVITIES UNDER DIFFERENT SAGITTAL}

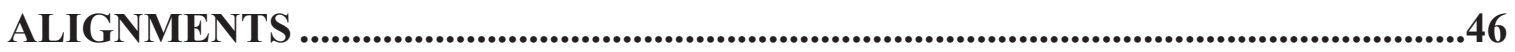

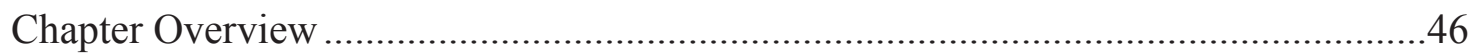

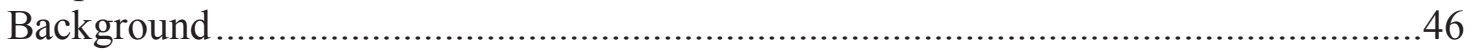

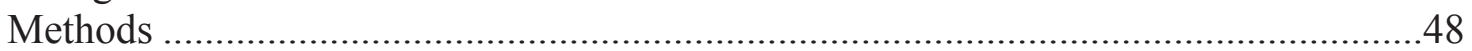

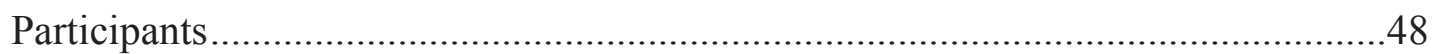

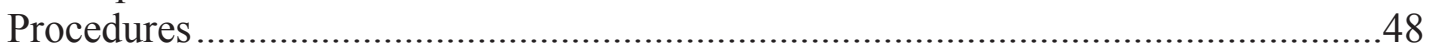

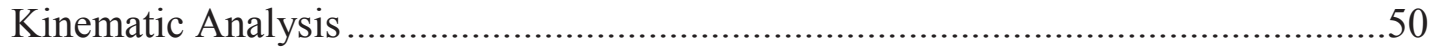

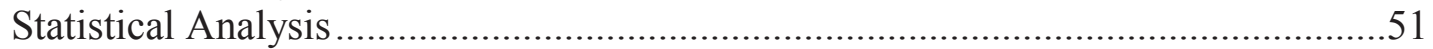

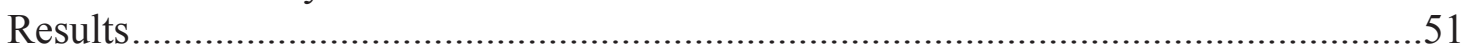

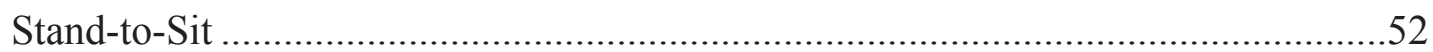

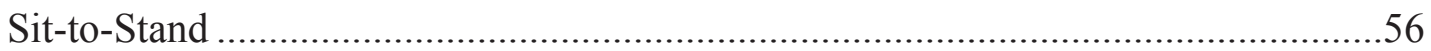

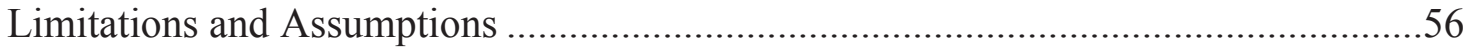

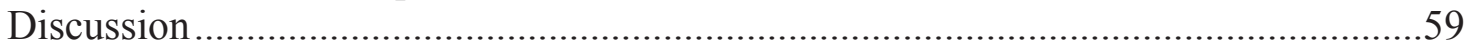

CHAPTER 6. GENERAL DISCUSSION.................................................................62

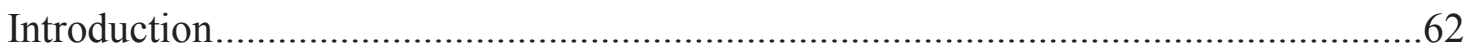

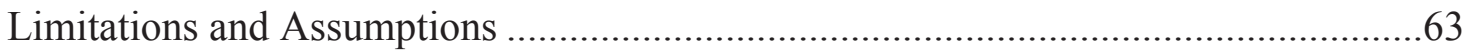

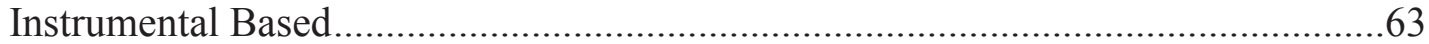

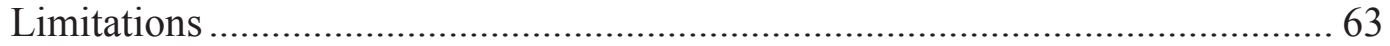

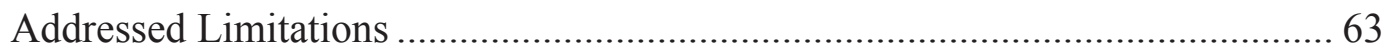

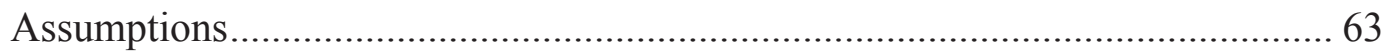

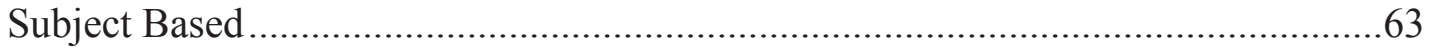

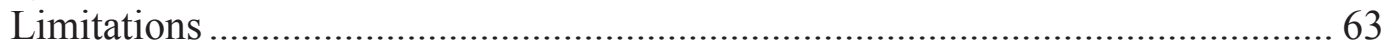

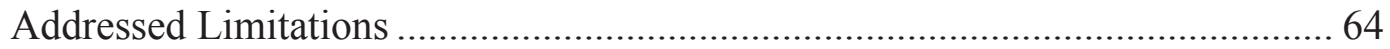

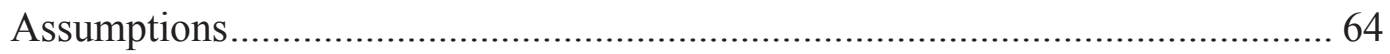

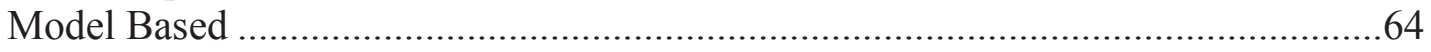

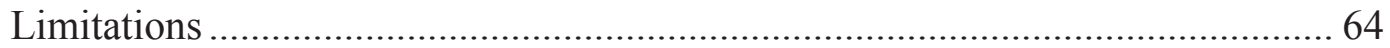

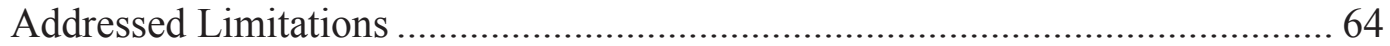

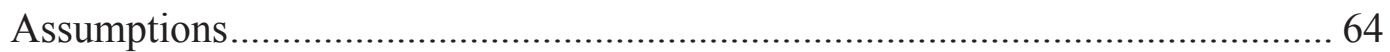

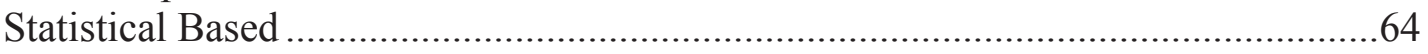

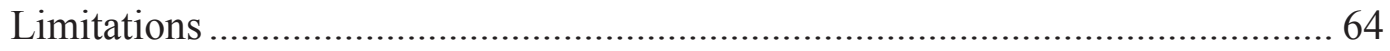

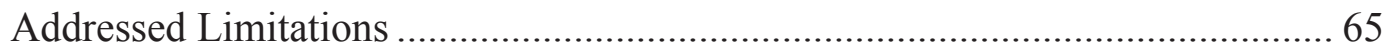

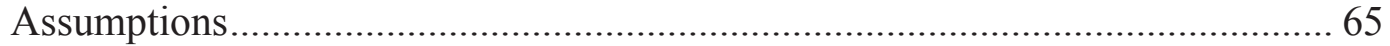

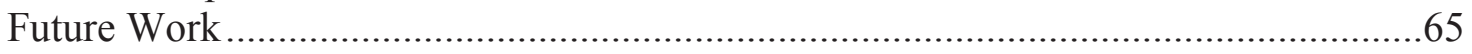

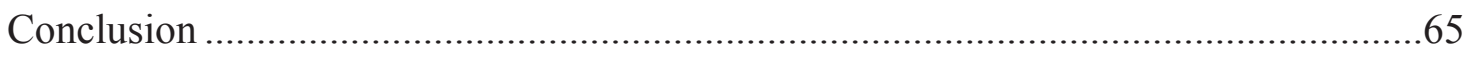

LIST OF REFERENCES ..................................................................................................67

APPENDIX A. TECHNICAL INSTRUMENT SPECIFICATIONS............................72 
Additional "Validation of Motion Analysis Spinal and Pelvic Measures Using an EOS X-ray System" Graphs

Additional "EOS Bi-planar X-ray Validates Hyper-Tensioned Clavicle Strap Alters

Lumbar Lordosis in Standing" Graphs .....

Additional "Spino-Pelvic Kinematics of Gait under Different Sagittal Alignments"

Graphs....

Additional "Spino-Pelvic Kinematics of Sit-to-Stand and Stand-to-Sit Activities under Different Sagittal Alignments" Graphs

APPENDIX C. NORMATIVE DATA TABLES .................................................83

APPENDIX D. SUMMARY OF PRELIMINARY INVESTIGATIONS .................84

APPENDIX E. INSTITUTIONAL REVIEW BOARD APPROVAL........................86

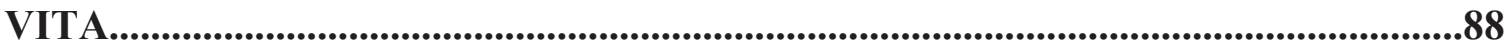




\section{LIST OF TABLES}

Table 1-1: $\quad$ Summary of Force Plate Specifications …..............................................15

Table 2-1: $\quad$ Summary of Subject Demographics (Mean \pm SD) ...................................19

Table 2-2: $\quad$ Summary of Lumbar Lordosis and Pelvic Tilt Changes Evaluated in

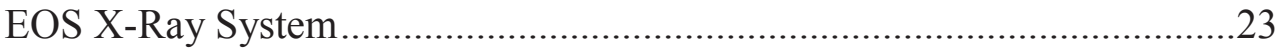

Table 2-3: $\quad$ Summary of Plumbline and Thoracic Kyphosis Changes ..........................24

Table 2-4: $\quad$ Summary of Bland-Altman and Regressions for Marker vs

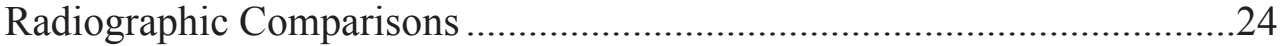

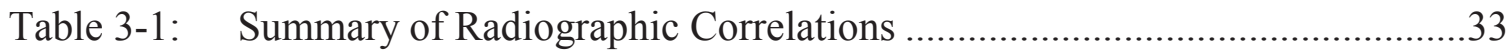

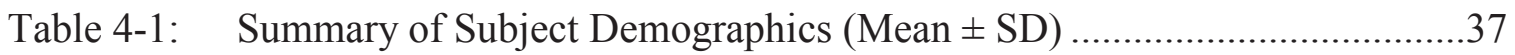

Table 4-2: $\quad$ Changes Decreased Lumbar Lordosis from Normal during Gait ................41

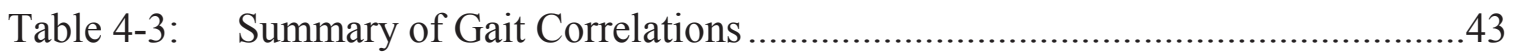

Table 5-1: $\quad$ Summary of Subject Demographics (Mean \pm SD) …...............................48

Table 5-2: Cycle Boundary Conditions ............................................................ 51

Table 5-3: $\quad$ Normal Values at Peak Hip Flexion during Stand-to-Sit and Sit-to-

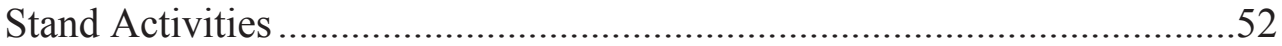

Table 5-4: Changes from Normal in Sit-to-Stand and Stand-to-Sit Activities at Peak Hip Flexion...........................................................................53

Table 5-5: $\quad$ Summary of Correlations during Stand-to-Sit Activities............................54

Table 5-6: $\quad$ Summary of Correlations during Sit-to-Stand Activities............................57

Table D-1: Summary of Changes of Lordosis and Anterior Pelvic Tilt from Normal ..85 


\section{LIST OF FIGURES}

Figure 1-1: $\quad$ Subjects with Flatback Deformity Following Spine Fusion Surgery ............2

Figure 1-2: Acetabular Component Orientation .............................................................

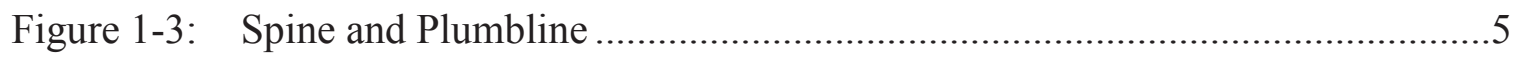

Figure 1-4: Pelvic Anatomy..................................................................................

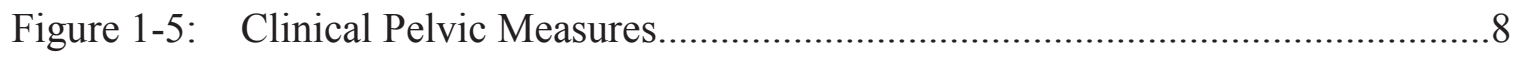

Figure 1-6: Clinical Spine Measures ........................................................................

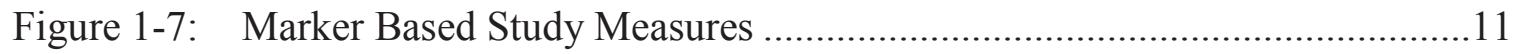

Figure 1-8: Graphic of Camera Setup.................................................................... 13

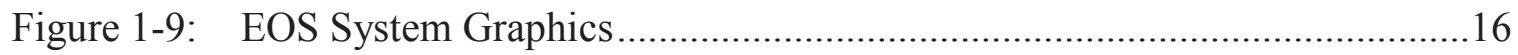

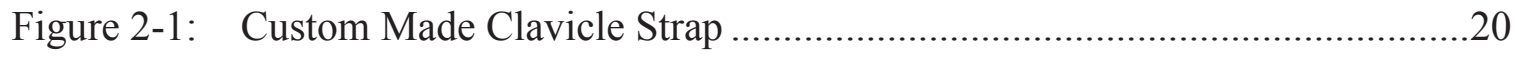

Figure 2-2: Bland-Altman Plots of Marker vs EOS Derived Lumbar Lordosis .............25

Figure 2-3: Marker vs Clinical Bland-Altman Plots for Thoracic Kyphosis and

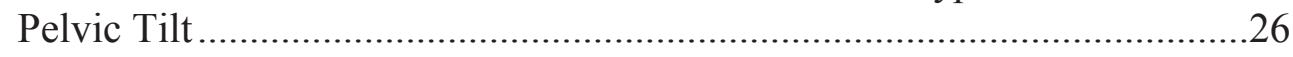

Figure 3-1: Individual Subject Responses to Orthotic..............................................33

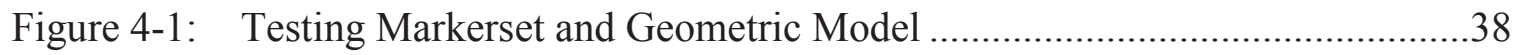

Figure 4-2: $\quad$ Significant Correlations during Gait ....................................................42

Figure 5-1: $\quad$ Significant Correlations during Stand-to-Sit at Peak Hip Flexion...............55

Figure 5-2: $\quad$ Significant Correlations during Sit-to-Stand at Peak Hip Flexion................58

Figure 5-3: Changes in Pelvic Tilt and Lumbar Lordosis Regressions during Sit-toStand.

Figure A-1： AMTI OR6-7-2000 Forceplate Technical Specifications...........................72

Figure B-1: Bland-Altman Plots of Changes in Plumbline and Clinical Pelvic Tilt

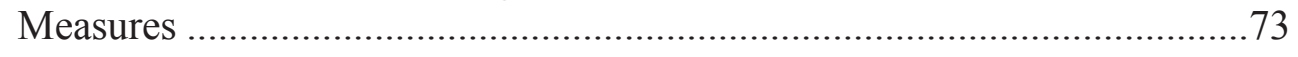

Figure B-2: Bland-Altman Plots of and Radiographic vs Pelvic Tilt Measures .............74 
Figure B-3: Linear Regression of Change in Radiographic Pelvic Tilt vs Change in T1/T12 Cobb Angle

Figure B-4: Change in Pelvic Tilt Correlations during Gait Correlations.........................76

Figure B-5: Change in Hip Flexion Correlations during Gait .........................................77

Figure B-6: Change in Pelvic Tilt and Hip Flexion vs Thoracic Kyphosis

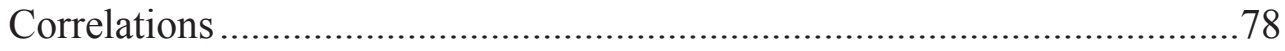

Figure B-7: Stand-to-Sit Change in Pelvic Tilt Correlations at Peak Hip Flexion ..........79

Figure B-8: Stand-to-Sit Changes in Hip Flexion Correlations at Peak Hip Flexion .....80

Figure B-9: Sit-to-Stand Changes in Pelvic Tilt Correlations at Peak Hip Flexion.........81

Figure B-10: Sit-to-Stand Changes in Hip Flexion Correlations at Peak Hip Flexion .....82

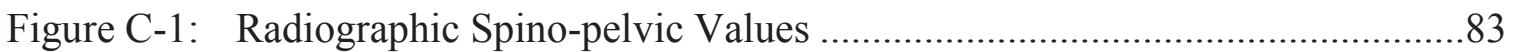




\section{LIST OF ABBREVIATIONS}

ACR

AMTI

AP

ASIS

BMI

CALC

DDD

DOF

DRSM

F1-3

FSI

GRF

GTOE

HRQOL

HS

LATFEMCON

LATMALL

LED

LHS

LL

NIR

MEDFEMCON

MEDMALL

ML

OA

PA

PSIS

RHS

RTH

RTIB

SACR

SD

SEM

Si-St

SFS

SR

$\mathrm{St}-\mathrm{Si}$

TO

THA

TK

UTHSC

$5^{\text {th }}$
Acromium

Advanced Mechanical Technology Inc.

Anterioposterior

Anterior Superior Iliac Spine

Body Mass Index

Calcaneus

Degenerative Disc Disease

Degree of Freedom

Dorsum

Fill marker 1-3

Fixed Sagittal Imbalance

Ground Reaction Force

Great Toe

Health-Related Quality of Life

Heel Strike

Lateral Femoral Epicondyle

Lateral Malleolus

Light Emitting Diode

Left Heel Strike

Lumbar Lordosis

Near-Infrared

Medial Femoral Epicondal

Medial Malleolus

Mediolateral

Osteoarthritis

Posterioanterior

Posterior Superior Iliac Spine

Right Heel Strike

Right Thigh Cluster

Right Tibial Cluster

Sacrum

Standard Deviation

Standard Error of the Mean

Sitting-to-Standing

Spinal Fusion Surgery

Right Sacrum

Standing-to-Sitting

Toe Off

Total Hip Arthroplasty

Thoracic Kyphosis

University of Tennessee Health Science Center

Fifth Metatarsal Head 


\section{CHAPTER 1. INTRODUCTION}

\section{Significance of Research}

Americans are undergoing spinal fusion surgery (SFS) at an ever increasing rate; in 2008 over 400,000 Americans underwent SFS with a national cost of approximately $\$ 33.9$ billion [1]. SFS is performed to alleviate pain and attempts to improve function in pathologies including degenerative disk disease and disk displacement [1-7]. Unfortunately, this treatment can result in abnormal spinal alignments [2-13] resulting in flatback deformities or fixed sagittal imbalance (FSI). Abnormal alignment then alters the position of the pelvis and acetabulum, decreasing the function and generating uncharacteristic hip contact patterns $[2,5,6,8,14-19]$.

During SFS the surgeon lays the patient prone and fuses together vertebrae adjacent to the pathological disk(s). In this prone position the surgeon can effectively maintain normal coronal alignment of the spine [4]. However, it is difficult for the surgeon to properly align the spine as viewed sagittally [3, 4]. Alterations of sagittal plane alignment as a result of pathology or surgery create changes in lumbar lordosis (LL) $[2-13,19]$. These postural alterations cascade to affect position and function of the lower extremities including the pelvis, hips, knees, and ankles [2, 5, 6, 8, 14, 19]. For example, SFS resulting in a flatback (Figure 1-1) deformity would cause a patient to compensate by flexing their hips, knees, and/or ankles to stand upright $[2,5,6,8,19]$.

Several studies have shown that patients with spinal pathology, such as degenerative disc disease, often have a coexisting hip pathology or subsequently develop hip pathology $[15,16]$. As the lumbar lordosis increases or decreases the forward tilt of the pelvis will compensate. Alterations in pelvic tilt due to spinal pathology will alter the contact pattern and femoral head coverage area of the hip joint $[14,20]$. This change in contact location will apply forces to cartilage not conditioned for such loads while changing femoral head coverage area will alter pressure distribution within the joint [21, 22]. Older or weakened cartilage, such as early stage arthritis, cannot adapt to new loading conditions and the cartilage will degrade more rapidly resulting in osteoarthritis (OA) $[21,22]$. People with hip OA often require surgical intervention, including total hip arthroplasty (THA), to alleviate pain and improve function.

When surgeons perform THA a new acetabulum is typically placed in the "safe zone" which is 5-25 deg of anteversion and 40-45 deg of inclination (abduction) [17, 20]. Acetabular anteversion is defined as the rotation towards the (ventral surface) midline of the body in the transverse plane (Figure 1-2A). Acetabular inclination is defined as rotation of the cup in the coronal plane away from the horizontal line and towards the midline (Figure 1-2B). In the "safe zone" the hips will have desired performance with a lower risk of dislocation, femoral impingement on the acetabular rim or excessive implant wear $[17,18,20]$. Patients who undergo SFS in the presence of a previous THA risk altered pelvic position. If anterior pelvic tilt decreases, acetabular anteversion will increase $[14,23]$ and create problems associated with deviation from the safe zone. 

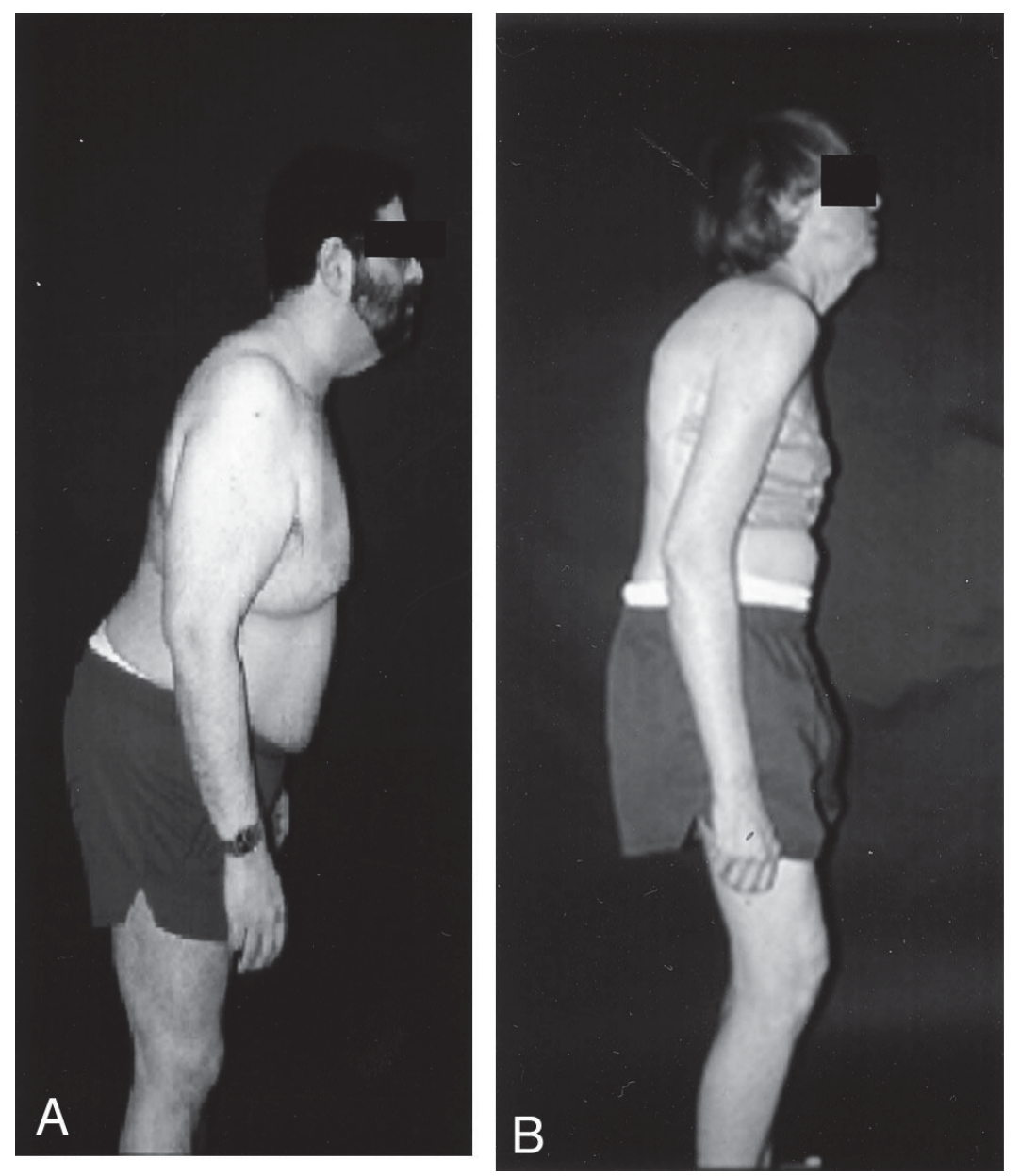

Figure 1-1: Subjects with Flatback Deformity Following Spine Fusion Surgery A) Non-compensated flatback deformity B) Flatback deformity compensated with flexed knees, hips, and ankles.

Source: Reprinted with kind permission from Sarwahi, V., O. Boachie-Adjei, S. I. Backus, and G. Taira. "Characterization of Gait Function in Patients with Postsurgical Sagittal (Flatback) Deformity: A Prospective Study of 21 Patients." Spine (Phila Pa 1976) 27, no. 21 (Nov 1 2002): 2328-37. 
A)

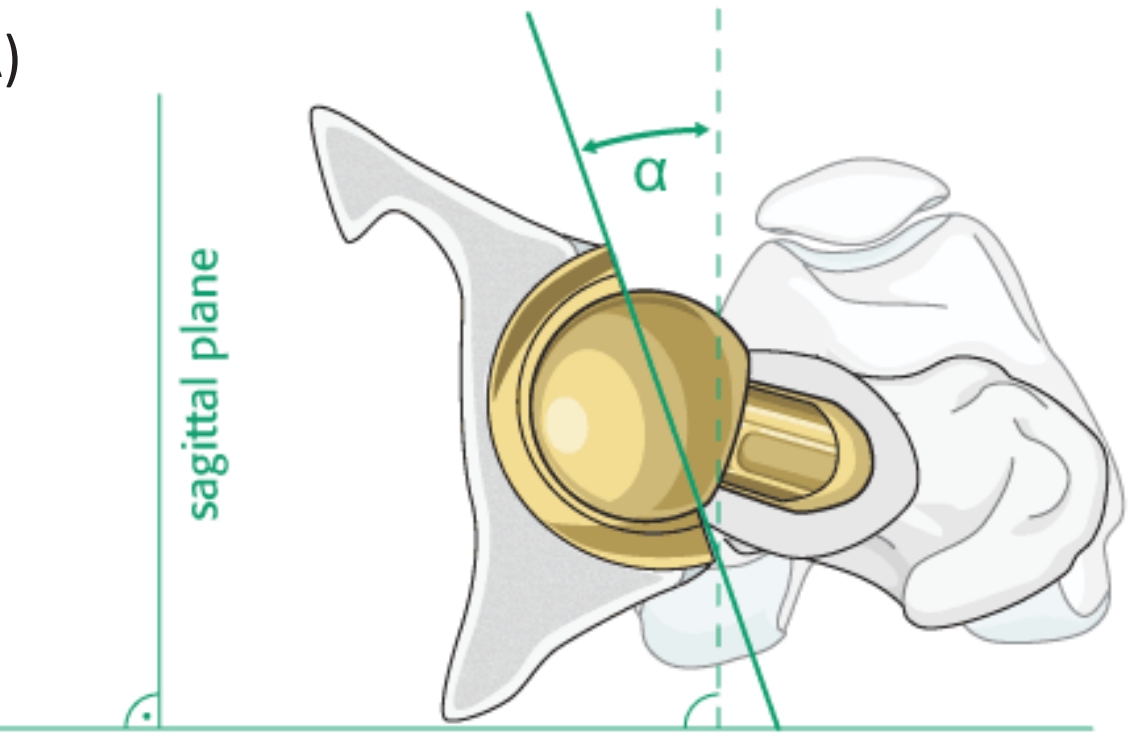

coronal plane

$$
\alpha=\text { Acetabular anteversion }
$$

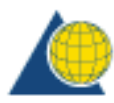

B)

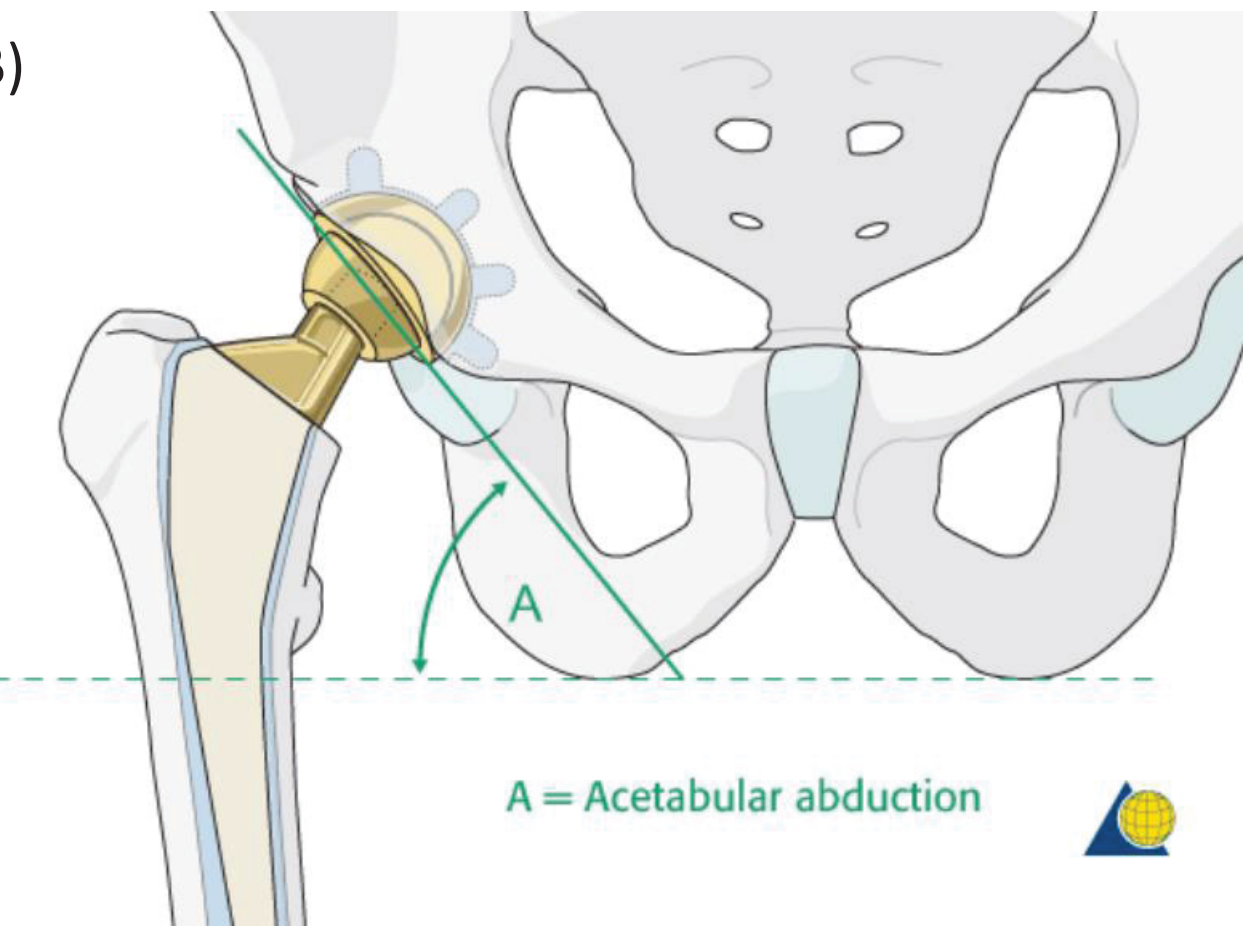

Figure 1-2: Acetabular Component Orientation

A) Acetabular Anteversion B) Acetabular Abduction/Inclination

Source: Reprinted with kind permission from AO Surgery Reference, www.aosurgery.org, accessed 6/24/2014. Copyright by AO Foundation, Switzerland. 


\section{Thesis Aim and Outline}

The goal of this study was to examine the influence of altered sagittal lumbar lordosis on sagittal pelvis kinematics during static stance, gait, sit-to-stand (Si-St), and stand-to-sit ( $\mathrm{St}-\mathrm{Si}$ ) by the use of a hyper-tensioned clavicle strap. We hypothesized that a subject would compensate an altered lumbar lordosis by altering their pelvic tilt and torso alignment to maintain a normal plumbline.

To investigate this hypothesis we utilized three-dimensional motion capture, and bi-planar X-ray technology to evaluate sagittal lumbar lordosis, pelvic tilt, and plumbline in ten young, healthy, subjects. These parameters were evaluated in a paired fashion, by comparing each subject with and without a hyper-tensioned clavicle strap. Static evaluation of these parameters were measured in a motion analysis laboratory, and validated using a state of the art standing bi-planar X-ray imaging system (EOS). Dynamic measurements of the lumbar spine and pelvis utilized a three dimensional motion analysis laboratory.

This thesis is composed of five chapters. Chapter 1 provides a brief overview of the anatomy of the spine and pelvis, as well as the instruments used. Chapter 2 shows the agreement of spinal measures derived from markers placed on the skin to their anatomical derived counterparts using an EOS X-ray system. Chapter 3 describes the procedure and kinematics of the spine and pelvis of subjects with a mechanically altered lumbar lordosis performing Si-St and St-Si. Chapter 4 similarly describes the procedure and kinematics of the spine and pelvis of subjects during gait with a mechanically altered lumbar lordosis during gait. Chapter 5 discusses the overarching clinical implications, limitations, and potential avenues of future study.

\section{Overview of Pelvic and Spinal Anatomy}

\section{Spine}

The spine is a collection of vertebrae structured in a column, connecting the skull to the pelvis. The spine is composed of 33 vertebrae forming 5 distinct structures: cervical, thoracic, lumbar, sacrum, and coccyx. While the vertebrae forming the cervical, thoracic, and lumbar can articulate independently, the five vertebrae of the sacrum are fused together, as are the remaining 3-4 vertebrae forming the coccyx (Figure 1-3A).

Each of the articulating vertebrae is wedge shaped, to a unique degree, giving the spine its characteristic s-shape when viewed sagittally (from the side). This shape can greatly influence physical function, motion, and balance $[2,3,5,6,8,14,19,24,25]$. The thoracic region has a distinct arc, while the lumbar has an equally defined inverted arc, termed kyphosis and lordosis respectively. These are the curves we are evaluating in this study. A spine is determined to be in good balance (i.e. a normal amount of kyphosis and lordosis) when the vertical line drawn from the center of the $7^{\text {th }}$ cervical vertebra $(C 7)$ to 
A)

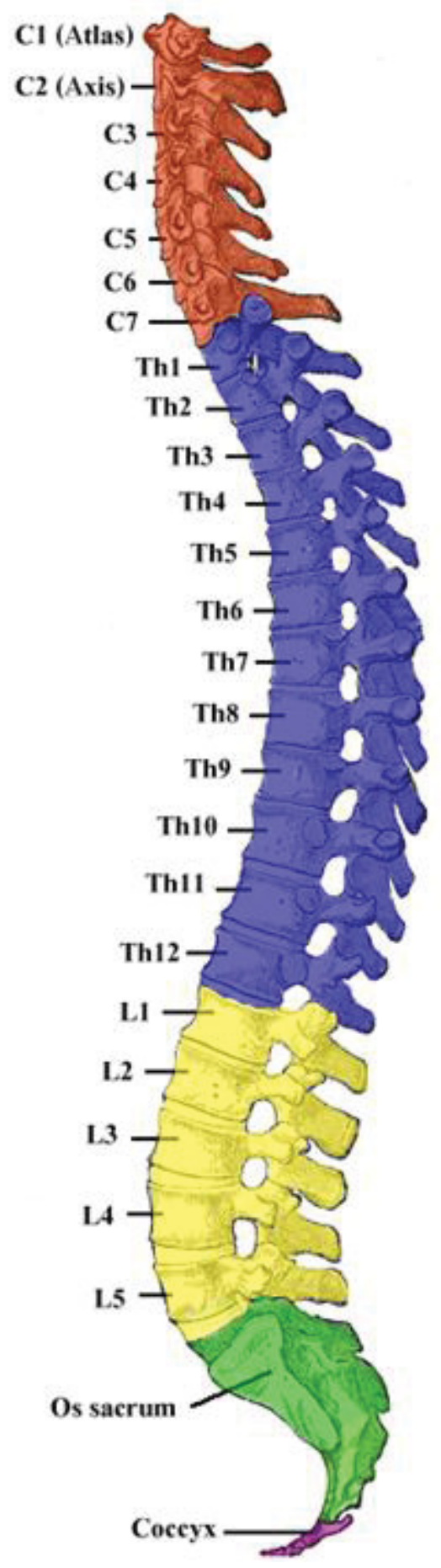

B)

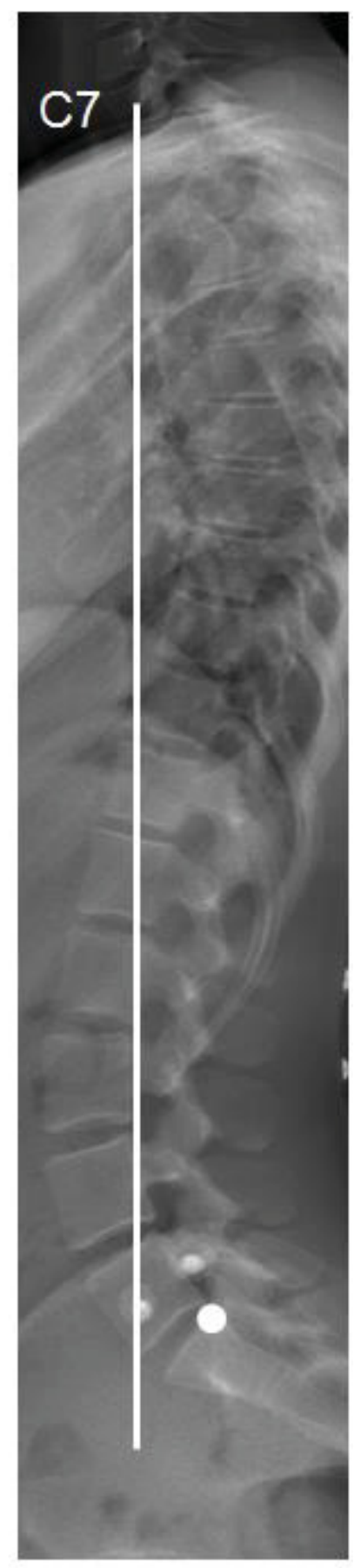

Figure 1-3: $\quad$ Spine and Plumbline

A) Representation of human spinal column B) Example of plumbline

Figure 1-3A Source: Reprinted with kind permission from Wikipedia, the free

encyclopedia: http://commons.wikimedia.org/wiki/File:Gray_111_-_Vertebral_columncoloured.png accessed 7-14-2014 
the ground passes within $3 \mathrm{~cm}$ of the posterior (back) corner of the sacrum (Figure 1-3B) [2-4]. This characteristic is termed sagittal vertical axis or plumbline and is an important measure in determining normal functionality. Plumbline can be shifted forward (anteriorly) by factors such as old age or from spine fusion surgery of the lumbar [2-13].

\section{Pelvis}

The bony structure of the pelvis is made up of the two hip bones, sacrum, and coccyx. The sacrum and coccyx were previously described as part of the spine, and their primary function is to transfer loads to the hip bones, and to serve as anchoring points for muscles. The two hip bones (left and right) are composed of the ilium, ischium, and pubis. The three structures come together to define the acetabulum (hip joint) and contain bony structures used to define pelvic motion. The ilium is the fan shaped bony structure most superior on the hip. The top ridge of the ilium is called the iliac crest and spans from the anterior superior iliac crest and posterior superior iliac crest Figure 1-4. The lower portion of the ilium also forms the superior portion of the acetabulum. The ischium forms the posterior of the acetabulum, and the pubis forms the remaining anterior structures.

As illustrated in Figure 1-4 the cartilage of the acetabulum neither fills the entire space nor forms a ring, but instead forms a horseshoe along the upper edge. The hole in the middle is to allow for the ligament which connects the head of the femur to the hip joint. The empty part of the horseshoe is filled with a fat pad. The contact area of the femoral heads is made larger by a ring of cartilage (labrum) that extends from the acetabular rim to beyond the radial apex of the femoral head. The labrum, functions to deepen the acetabulum, aids in holding the femoral head in the joint socket, and provides cushion for when the femoral neck impinges on the acetabular rim.

Deformities or pathologies that shift the forward tilt of the pelvis concurrently alter the contact of the femoral head with the acetabulum [14, 23]. With the contact location changed, cartilage at that location may not be conditioned to the applied loads $[21,22]$. If the cartilage cannot adapt it will degrade and lead to the development of osteoarthritis $[21,22]$. Overall, changing the forward tilt of the pelvis can lead to the development of osteoarthritis or decreased hip function.

\section{Overview of Pelvic, Spine, and Study Measurements}

Clinical measurements of anatomical structures often depend on accessibility and visibility of the structures during activities such as surgery, physical therapy, or motion

analysis. As a result, multiple techniques have been developed to measure pelvic tilt and lumbar lordosis. 


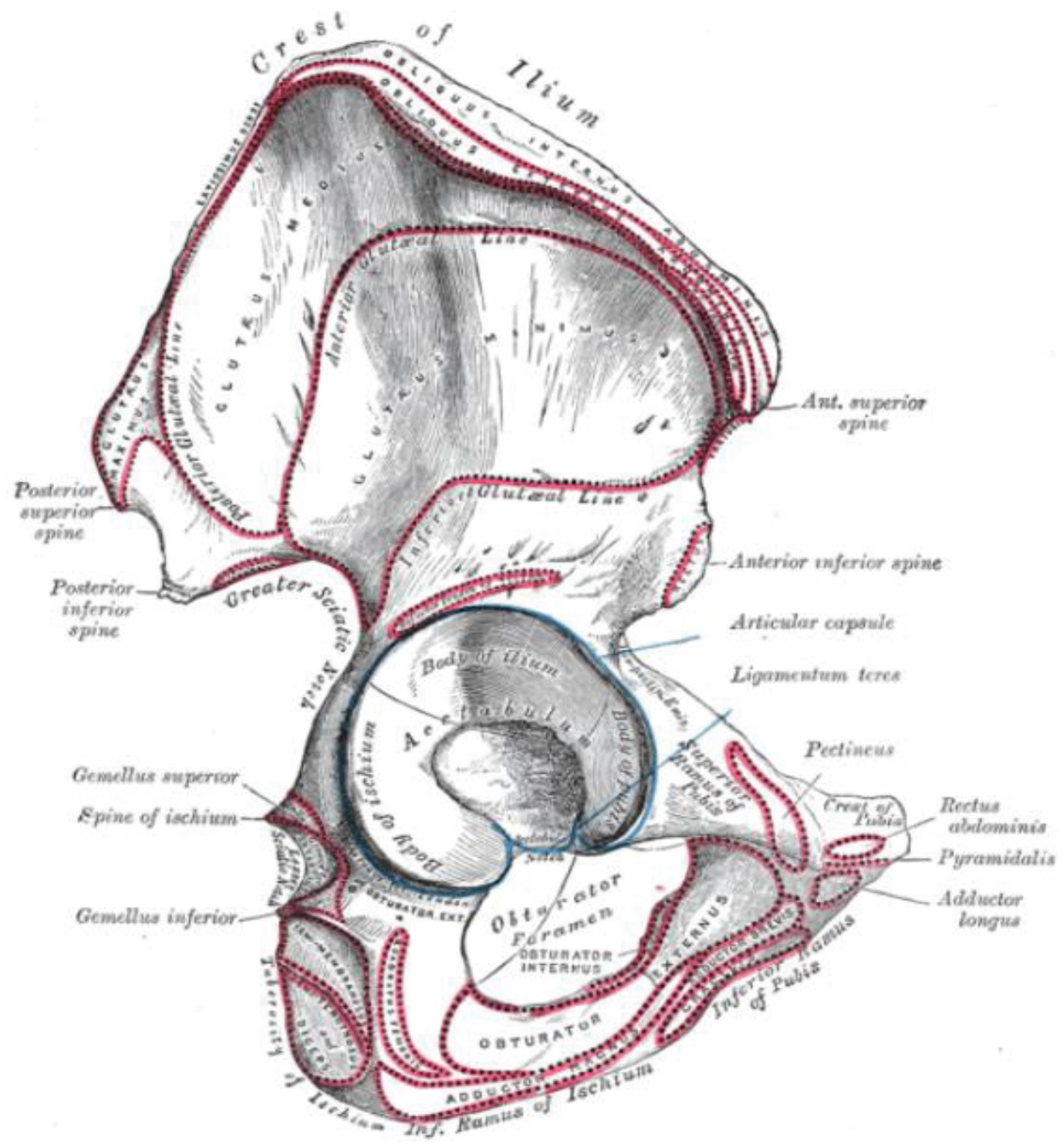

Figure 1-4: Pelvic Anatomy

Source: Reprinted with kind permission from Wikipedia, the free encyclopedia http://en.wikipedia.org/wiki/File:Gray235.png accessed 2-19-2014 


\section{Pelvic Measures}

Three common measures exist to represent pelvic tilt resulting from the limitations of orthopaedic surgery, standing radiographs, and physical therapy. During orthopaedic surgery, when a patient is supine, pelvic tilt is defined by the angle formed by the anterior pelvic plane (APP). The APP is generated by connecting the anterior superior iliac spines (ASISs) to the pubic symphysis and the horizontal plane [14]. During standing radiographs, pelvic tilt may be evaluated as the angle formed by the line segment connecting the middle of the sacral endplate to the center of the bicoxofemoral axis (axis passing through the geometric center of the femoral heads) and the vertical plane $[3,14,26,27]$. This measure of pelvic tilt is termed "radiographic pelvic tilt" for this study. The pelvic tilt typically used in physical therapy and motion analysis is the angle formed by the line segment connecting the ASISs and poster superior iliac spines (PSISs) as referenced from horizontal when the subject is in the standing position [28, 29]. An illustration of these various measures can be found in Figure 1-5A.

Referring to these three pelvic tilt measurements interchangeably assumes that the pelvis rotates about the bicoxofemoral axis, the pelvis is one solid structure, there is no or little sacro-iliac motion, no morphological differences exist between subjects, and the pubic symphysis-ASIS-PSIS angle is $90 \mathrm{deg}$. When operating in the sagittal plane, the first two assumptions are reasonable. No sacro-iliac motion may be considered valid in operating conditions where the subject is anesthetized. Other times the passive motion of the sacroiliac joint has been suggested to be as much as $10 \mathrm{~mm}$ in healthy adults [30]. Morphological differences alone can vary pelvic tilt as much as 23 deg if comparing the ASIS-PSIS tilt to the anterior pelvic plane [29]. By looking only at within subject changes in pelvic tilt, the morphologic variations are negated and it is possible to compare changes between the various measures of pelvic tilt.

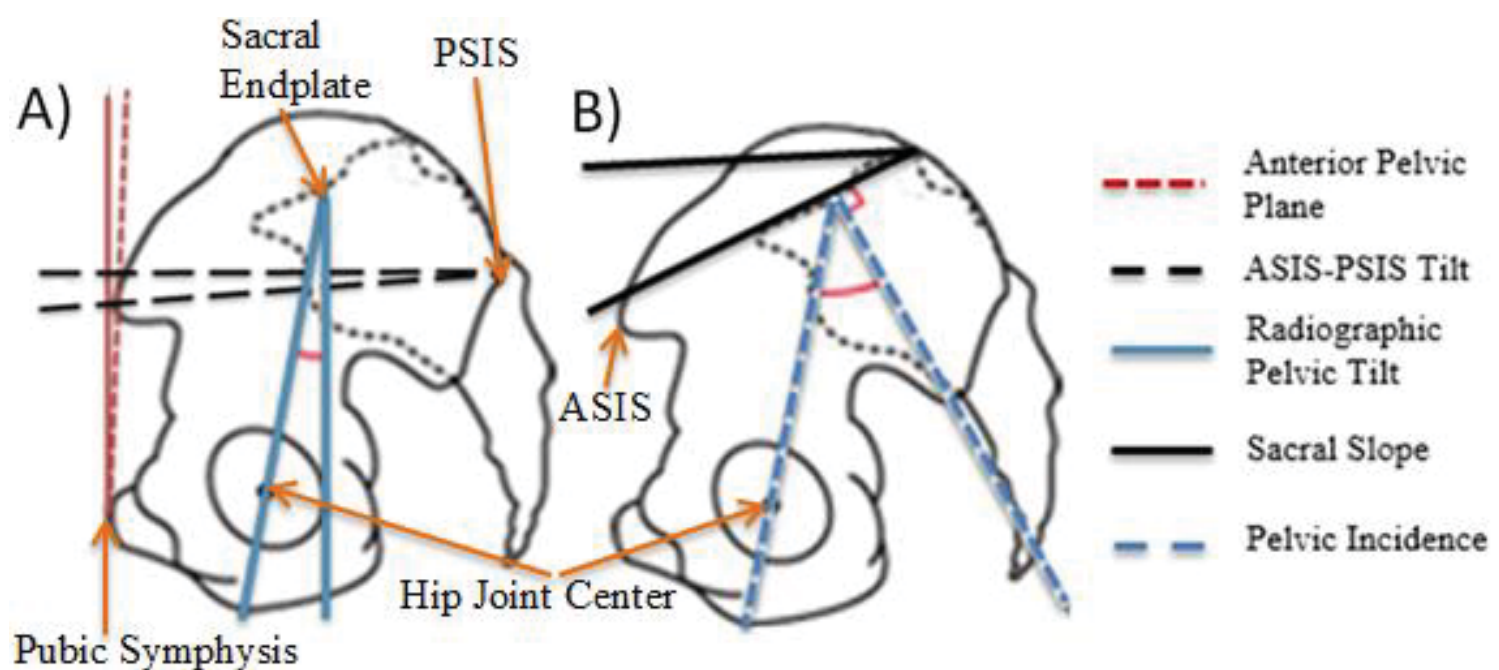

Figure 1-5: Clinical Pelvic Measures

A) Measures of pelvic tilt B) Measures of sacral slope and pelvic incidence. 
Other common pelvic measures are pelvic incidence and sacral slope Figure 15B. Sacral slope is the angle the sacral endplate forms with a horizontal reference line. Pelvic incidence is the angle formed by the line segment connecting the bicoxofemoral axis to the midpoint of the sacral endplate and the orthogonal line to, and passing through the midpoint of, the sacral endplate Figure 1-2B. Notice there is a geometric relationship between sacral slope, radiographic pelvic tilt, and pelvic incidence (Equation 1-1):

$$
\text { Pelvic Incidence }=\text { Sacral Slope }+ \text { Pelvic Tilt }
$$

These measurements are commonly used in surgery and several studies suggest that pelvic incidence and sacral slope are strong predictors of patient specific normal LL [27, $31]$.

\section{Spine Measures}

The gold standard of thoracic and lumbar measures requires visualization of the endplates of the vertebrae to generate a Cobb angle, as is done in standing radiographs. A Cobb angle is the acute angle formed by the orthogonal lines extending from the vertebral endplates spanning the region of interest. LL can be defined as either the Cobb angle in the sagittal plane of the superior L1 and inferior L5 endplates [3], or as the Cobb angle of the superior L1 endplate and the superior sacral endplate (Figure 1-6A) [4, 8, 27, 32]. Similarly, thoracic kyphosis (TK) is defined as either the Cobb angle of the inferior T12 endplate and the T4 [26, 27, 32] or T1 superior endplates [32] (Figure 1-6B).
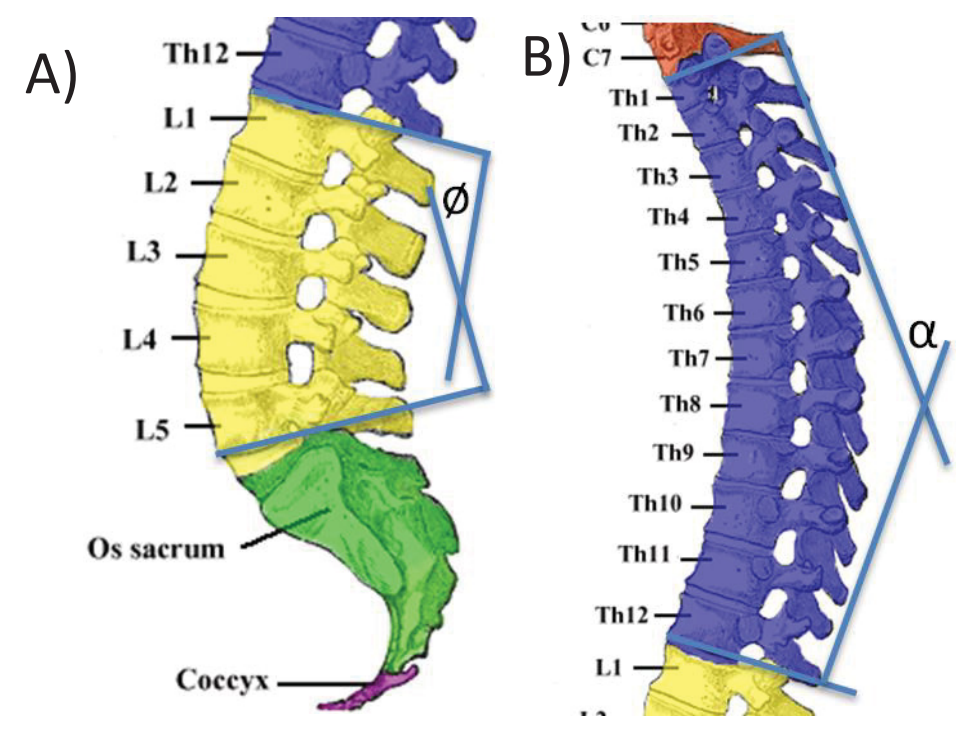

Figure 1-6: Clinical Spine Measures

A) Graphic of L1-L5 Cobb angle B) Graphic of T1-T12 Cobb angle

Source: Modified with kind permission from Wikipedia, the free encyclopedia:

http://commons.wikimedia.org/wiki/File:Gray_111_-_Vertebral_column-coloured.png accessed 7-14-2014 
The vertebral endplates are not visible during motion capture analysis thus skin mounted retro-reflective markers are placed over bony landmarks, such as spinous processes or adjacent muscle bellies, to track spine motion. Studies using magnetic resonance imaging and radiographic techniques have shown skin mounted markers correlate strongly with radiographic position and angle measures [33, 34]. In nine healthy young males, Mörl et al (2006) found skin motion related marker shift during flexion was not significantly different from zero for either markers placed over the muscle bellies or spinous processes; and further determined that skin motion was several times less for markers placed over the spinous processes than those placed over the muscle bellies ( median $=0.38 \mathrm{~mm}$ vs $0.86 \mathrm{~mm}$ ) [34]. A collection of techniques involving retro-reflective markers have been used to describe the spine including lumbar depth [35], arc fitting [35], plumbline [36], as well as a variety retro-reflective marker locations and configurations [33-35, 37-39]. However, a standard has not yet been established. As a result, it is often difficult to compare absolute LL or TK measures between studies. For this reason, the current study reported angle differences and ranges of motion.

\section{Study Measures}

This study used retro-reflective markers placed on the skin to track and measure body segments. The primary measures of interest included pelvic tilt, hip flexion, plumbline, LL, TK, and trunk-pelvic angle. The marker placement and visualization of these measures are illustrated in Figure 1-7A-C.

The pelvic tilt and plumbline measures were measured with respect to markers placed over the anatomical landmarks of their clinical counterparts. For pelvic tilt, markers were placed over the ASISs and PSISs, and the line segment connecting the midpoints of the ASISs and PSISs was referenced from the horizontal line extending from the midpoint of the PSISs Figure 1-7A. To measure plumbline, markers were placed over the spinous process of $\mathrm{C} 7$ and S2. The horizontal displacement of the $\mathrm{C} 7$ marker from S2 serves as our plumbline measure Figure 1-7B.

By measuring the angle formed by the $\mathrm{C} 7$ marker and the midpoints of the ASISs and PSISs, it is possible to measure the angle of the trunk relative to the pelvis. This angle was unimaginatively termed trunk-pelvic angle for this study Figure 1-7A, and revealed if the trunk and pelvis rotate as a whole about the bicoxofemoral axis or independently. With the edition of the pelvic tilt measurement, it possible to isolate trunk motion and relate it to the vertical or horizontal axis.

Given the end goal of this study was to relate changes in the lumbar to changes in the hip, it was essential to measure hip flexion. Hip flexion, was represented by the angle formed by the line connecting the hip joint center to the knee joint center as referenced from a line orthogonal to the ASIS-PSIS line of the pelvis Figure 1-7A. Estimating the position of the knee and hip joint centers was done by markers, and morphological algorithms. The knee joint center was defined as the midpoint of the line segment 


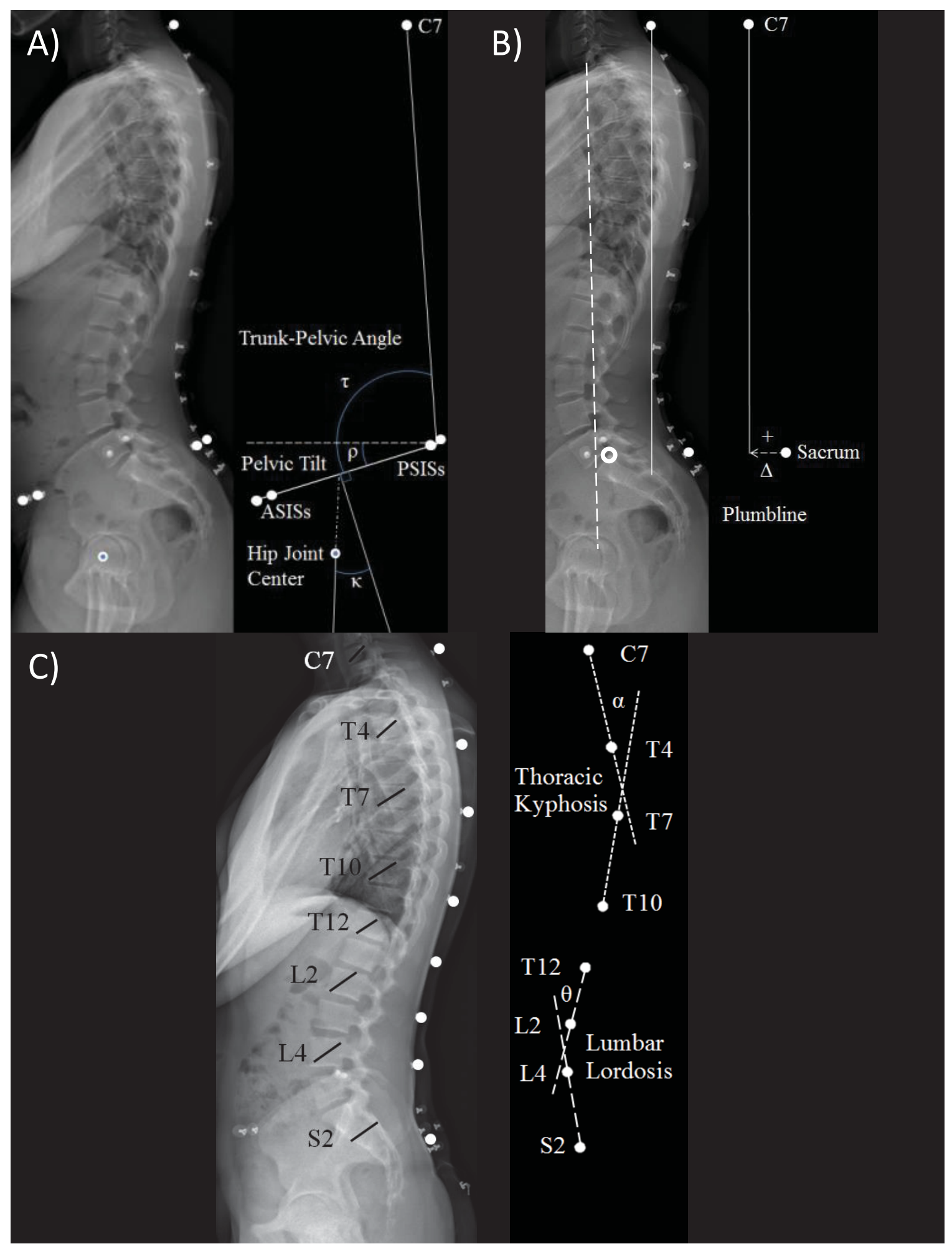

Figure 1-7: Marker Based Study Measures

A) Trunk-Pelvic angle, pelvic tilt, and hip flexion B) Plumbline, dashed represents clinical plumbline C) Thoracic kyphosis and lumbar lordosis 
connecting the markers placed on the medial and lateral epicondyles of the knee. The hip joint center was estimated by Equation 1-2, adapted from previous works [40, 41].

$$
\text { Hip Joint Center }(\mathrm{x}, \mathrm{y}, \mathrm{z})=( \pm 0.36 * \text { ASIS, }-0.19 * \text { ASIS, }-0.3 * \text { ASIS })
$$

ASIS represents the distance of the ASIS marker, located nearest the predicted joint, from midpoint of the line segment connecting both ASISs.

TK and LL are estimated by 4 markers spanning the length of each curve. TK was measured by placing markers over the $\mathrm{C} 7, \mathrm{~T} 4, \mathrm{~T} 7$, and $\mathrm{T} 10$ spinous processes. The acute angle formed by the C7-T4 and T7-T10 lines served as our kyphosis measure. Similarly, the markers were placed over the T12, L2, L4, and S2 spinous processes to measure LL. The acute angle formed by the T12-L2 and L4-S2 lines then served to represent LL. This marker configuration and usage has been used in other studies [38, 39], but it has yet to be validated using radiographs. The hope behind a markerset of this nature is that the markers placed over the spinous processes will generate the lines forming the acute angles illustrated in Figure 1-3A and $\mathbf{B}$.

By considering these 6 parameters we believe we can adequately understand and interpret the motion and compensation mechanisms of the spine and pelvis during activities of daily living when under different postural conditions.

\section{Overview of Experimental Instruments}

\section{Qualysis Motion Capture System}

The University of Tennessee Health Science Center (UTHSC) Motion Analysis Lab has 10 opto-electronic cameras (Qualisys AB, Gothenburg, Sweden) consisting of models 100, 300, and 310 positioned about the room in a circular fashion surround a raised platform Figure 1-8. These cameras have the ability to record images both in the visible and infrared spectrum. The $3 \mathrm{xx}$ series have a 1.3 megapixel resolution while the 100 series has a 0.3 megapixel resolution. For this reason the lab has only three 100 series cameras. Each camera is equipped with a ring of near-infrared (NIR) light emitting diodes (LEDs) which emit a narrow band of infrared light (wavelengths between 0.75-1.4 $\mu \mathrm{m})$. These coupled with retro-reflective markers provide the ability to track motion at sub-millimeter resolution. Using the camera setup in Figure 1-8 for this study, marker position was on average calibrated to $0.4 \mathrm{~mm}$ resolution.

The retro-reflective markers used were typically $12.7 \mathrm{~mm}$ in diameter, $9.5 \mathrm{~mm}$ markers were used when little space was available on the segment of interest. The size of the marker used depended on the size of the segment to be tracked, the speed which it was expected to move, and the proximity of the cameras. If the markers were too small for the aforementioned conditions, the cameras could not track the markers and no position data will be recorded. Millimeter size markers have been used to track thumb 


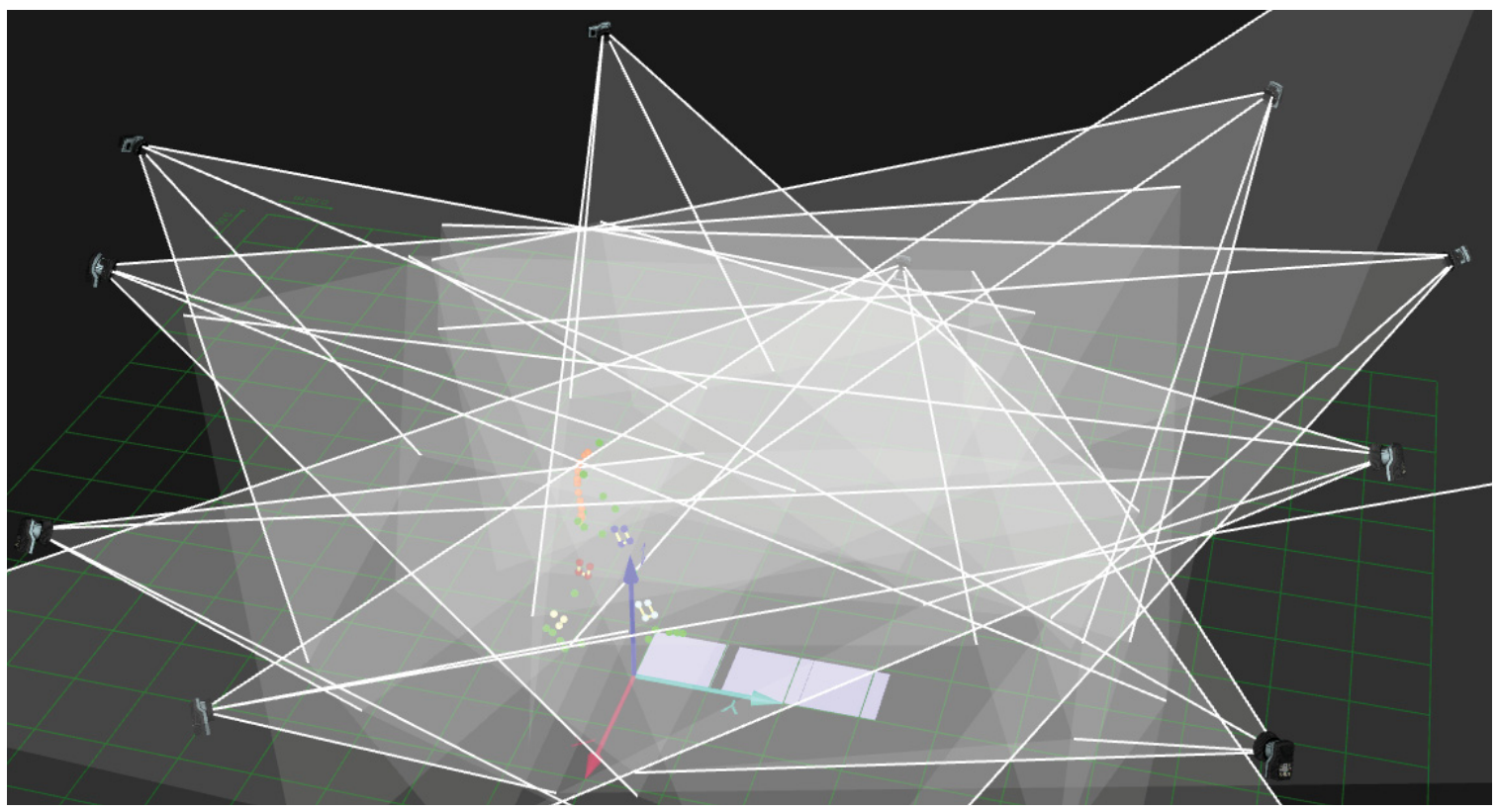

\section{Figure 1-8: Graphic of Camera Setup}

and finger motion with the cameras in close proximity, while markers several centimeters in diameter have been used during running activities. Each retro-reflective marker is covered in a material which reflects the infrared light emitted by the cameras. The cameras then collect the narrow wavelength of light. The light reflected from the markers is interpreted as a disk, and the geometric center of that disk represents the marker's location.

This form of motion tracking is commonly used to evaluate full body kinematics of living subjects. However, it comes with the limitation that markers are attached to the skin. The skin can move independently of the bone and muscle beneath it, especially in high body mass index individuals. This can create inaccuracies in motion as high as 10 deg in knee flexion [42]. For this reason studies are conducted with the aim to quantify and reduce this error [34, 42].

\section{AMTI Force Plates}

The Motion Analysis Lab is equipped with three instrumented force plates (AMTI, Watertown, MA, USA) Model OR6-7 2000 series. The force plates are embedded along a 25 foot-long platform in the middle of the motion capture volume. By measuring and recording ground reaction forces as well as segment motion during an activity it is possible to use inverse dynamics to estimate external joint moments and powers. In a similar fashion this method can be applied to estimate the changes in joint reaction forces due to ground reaction forces (GRF). However, changes due to GRF are small when compared to contraction and co-contraction forces exerted across a joint [43]. 
The force plates operate on the wheatstone bridge principle. A collection of strain gauges transduces the distributed load into changes in voltages representing forces and moments in a force plate specific $\mathrm{x}-\mathrm{y}-\mathrm{z}$ coordinate system ( $\mathrm{Fx}, \mathrm{Fy}, \mathrm{Fz}, \mathrm{Mx}, \mathrm{My}$ and $\mathrm{Mz}$ ). Based on the outputs of the strain gauges, equations:

$$
\begin{gathered}
\mathrm{COPx}=(\operatorname{Point}(\mathrm{z}, 0,0) * \mathrm{Fx}-\mathrm{My}) / \mathrm{Fz} \\
\mathrm{COPy}=(\operatorname{Point}(\mathrm{z}, 0,0) * \mathrm{Fy}+\mathrm{Mx}) / \mathrm{Fz} \\
\mathrm{COPz}=\operatorname{Point}(\mathrm{z}, 0,0)
\end{gathered}
$$

are used to estimate the point on the force plate where the applied ground reaction forces generate the equivalent moments about the origin of the force plate in all three axes. This point is then termed the center of pressure.

Provided in Table 1-1 is a summary of technical specification for the AMTI OR672000 series force plates used in the lab. A detailed table including specification such as sensitivities, and errors (non-linearity, hysteresis, and resolution) can be found in Appendix A (Figure A-1). Using a root mean squared approach the instrumentation error for the force components $(\mathrm{x}, \mathrm{y}, \mathrm{z})$ at full scale output $(4450 \mathrm{~N}, 4450 \mathrm{~N}, 8900 \mathrm{~N})$ is \pm $13 \mathrm{~N}(2.8 \mathrm{lbf}), \pm 13 \mathrm{~N}(2.81 \mathrm{bf})$, and $\pm 25 \mathrm{~N}$ (5.61bf), respectively.

\section{EOS Bi-planar X-ray System}

The EOS system (EOS Imaging, Cambridge, MA, USA) concurrently acquires posterior and lateral radiographs of a subject in a standing or seated position. It does this by scanning the subject from head to toe with two very thin fan-shaped X-ray beams positioned orthogonally to each other Figure 1-9A. Capturing both directions simultaneously ensures that anatomical structures, including vertebral bodies, are in the same position in both planes. This increases the accuracy of a $3 \mathrm{D}$ reconstruction by eliminating the possibility of the subject changing posture between radiographs.

The two radiographic images are then processed using a semi-automated 3D reconstruction protocol. Technicians trained with the software and familiar with bony structures mark the locations of key anatomical landmarks. For the spine, these landmarks consist of the 4 corners of each vertebra from the first thoracic to the fifth lumbar in both radiographs [44]. The software has access to a morphological data base of normal and scoliotic vertebrae. Using this database, and several interpolation algorithms, the program can estimate the 3D model of each vertebrae [45]. Even after generating a 3D model, technicians are still able to refine spinal contorts and vertebrae orientation.

During the reconstruction, a local coordinate system is generated for each vertebra. With this information it is possible to measure individual segment rotation, as well as Cobb angles describing the cervical, thoracic, and lumbar regions Figure 1-9B. The EOS program also generates an axis through the centers of the subject's left and right 
Table 1-1: $\quad$ Summary of Force Plate Specifications

\begin{tabular}{lll}
\hline \multicolumn{2}{c}{ Specifications (units) } & \multicolumn{1}{c}{ Values } \\
\hline Weight & $(\mathrm{N} / \mathrm{lbf})$ & $276.45 / 62$ \\
Dimensions [WxLxH] & $(\mathrm{mm} / \mathrm{in})$ & $464 \times 508 \times 83 / 18.25 \times 20 \times 3.25$ \\
Maximum Excitation Voltage & $(\mathrm{V})$ & 10 \\
Force Capacity [Fx-Fy-Fz] & $(\mathrm{N} / \mathrm{lbf})$ & $4,448-4,448-8,896 / 1,000-1,000-2,000$ \\
Moment Capacity [Mz-My-Mz] $(\mathrm{N}-\mathrm{m} /$ in-lbf) & $2258-2258-1129 / 20,000-20,000-10,000$ \\
\hline
\end{tabular}




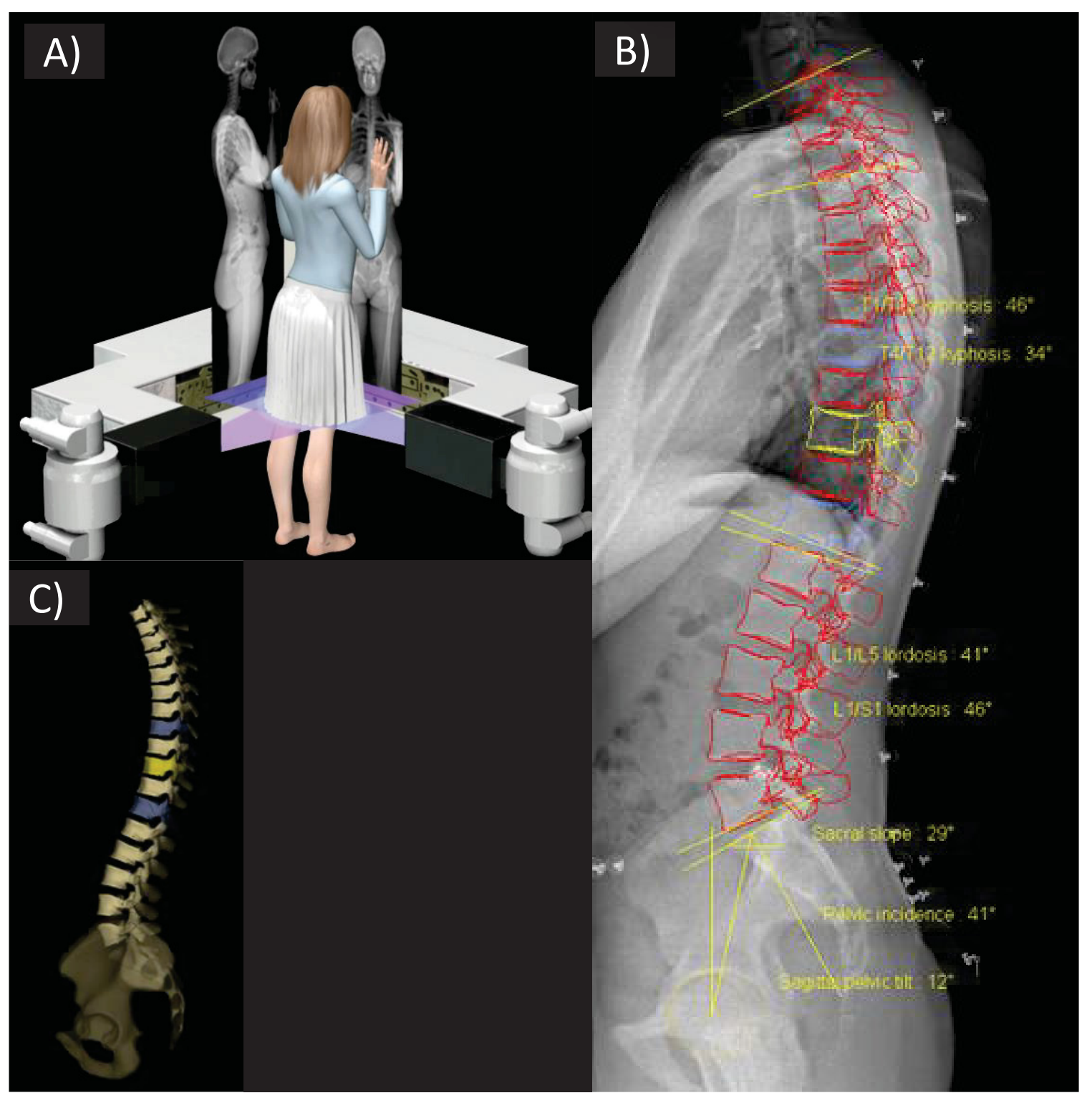

Figure 1-9: $\quad$ EOS System Graphics

A) Graphic of bi-planar x-ray system. B) Example of output from EOS system C) Generated 3D spine model using EOS system.

Figure 1-9A Source: Reprinted with kind permission from EOS Imaging:

http://www.eos-imaging.com/uploads/images/principe_acquisition.jpg accessed 2-18-14. 
acetabulum. The axis allows the program to predict "true" sagittal alignment and can report the sagittal angle in either the true sagittal or scanned sagittal plane. The whole process, scanning to completed reconstruction, takes less than an hour and generates images as shown in Figure 1-9C. 


\section{CHAPTER 2. VALIDATION OF MOTION ANALYSIS SPINAL AND PELVIC MEASURES USING AN EOS X-RAY SYSTEM}

\section{Chapter Overview}

The occurrence of spine and hip related pathologies in the United States continues to increase. As a result, many researchers aim to understand the motion of the spine and pelvis in living subjects using motion capture technology. No study prior to this has sought to relate the angle measures derived from retro-reflective markers placed over the spine and pelvis to clinical measures elicited from standing radiographs. This study utilized eight young, healthy subjects with no history of joint pain, or surgeries. The subjects were evaluated in a paired fashion using an EOS X-ray system in both a normal and posture altered condition, with retro-reflective markers adhered to the skin over the C7, T4, T7, T10, T12, L2, L4, S2 spinous processes as well as their ASISs and PSISs. Sagittal changes in spinal curvature and pelvic tilt derived from skin markers were compared to radiographic derived measures using the Bland-Altman method and linear regression. Changes in TK as measured by the acute angle formed by lines passing through the C7-T4 and T7-T10 markers had a $3 \pm 8^{\circ}$ (difference \pm 2 SD) bias compared to the T1/T12 Cobb angle. Changes in lumbar lordosis as measured by the acute angle formed by lines passing through the T12-L2 and L4-L2 markers had a $3 \pm 8^{\circ}$ and $0 \pm 9^{\circ}$ bias compared the L1/L5 and L1/S1 Cobb angles, respectively. The changes in ASISPSIS marker based pelvic tilt showed a $1 \pm 8^{\circ},-2 \pm 9^{\circ}$, and $0 \pm 5^{\circ}$, bias when compared the anatomical ASIS-PSIS tilt, anterior pelvic plane, and "radiographic pelvic tilt" (midpoint of the sacral endplate-hip joint center relative to vertical), respectively.

\section{Background}

Persons with spinal pathology, such as arthritis or degenerative disc disease, often have coexisting or subsequently develop hip pathology $[15,16]$. It is estimated that $18 \%$ of individuals undergoing total hip arthroplasty (THA) have concurrently developed a lumbar spine disorder [16]. If the lumbar disorder results in excessive pain or spinal deformation spine fusion surgery (SFS) is used to correct and treat the pathology [1-7]. In 2008 over 400,000 Americans underwent SFS with a national cost of approximately $\$ 33.9$ billion [1], and the rate Americans are undergoing SFS and THA is ever increasing [1]. As a result, there is a demand to further understand these conditions as well as the spine-pelvis relationship during activities of daily living.

Activities of daily living are investigated using motion capture technology to analyze kinetics and kinematics. Even though the spine has been investigated with this technology for over 30 years, no standard markerset exists to measure spinal motion using 3D motion analysis, and as a result it is difficult to compare lumbar lordosis (LL) or thoracic kyphosis (TK) measures between studies. Additionally, to the author's knowledge, no markerset has been validated with radiographic measures of LL and TK Cobb angles. 
Studies using magnetic resonance imaging and radiographic techniques have shown skin mounted markers correlate strongly with radiographic position and angle measures [33, 34]. In nine healthy young males, Mörl et al (2006) found skin motion related marker shift during flexion was not significantly different from zero for either markers placed over the muscle bellies or spinous processes; and further determined that skin motion was several times less for markers placed over the spinous processes than those placed over the muscle bellies (median $=0.38 \mathrm{~mm}$ vs $0.86 \mathrm{~mm}$ ) [34].

A standard markerset to measure pelvic kinematics in motion analysis utilizes markers placed over the ASISs and PSISs [28, 46, 47]. Pelvic tilt it then measured by referencing the ASIS-PSIS line to horizontal [28, 29]. However its relationship to the APP and radiographic pelvic tilt is not well understood. During THA the subject is supine and pelvic tilt is measured by the angle formed by the APP and horizontal. The APP is generated by connecting the ASISs and pubic symphysis [14]. During standing radiographs, pelvic tilt may be evaluated as the angle formed by the line segment connecting the middle of the sacral endplate to the center of the bicoxofemoral axis (axis passing through the geometric center of the femoral heads) and the vertical plane $[3,14$, $26,27]$. This measure of pelvic tilt is termed "radiographic pelvic tilt" for this study. The marker pelvic tilt has not be related to either the APP or radiographic pelvic tilt.

The purpose of this study is to validate the spinal markerset used by Fowler et al (2006) and Syczewska et al (2012), as well as marker derived pelvic tilt to other clinical measures of pelvic tilt. Understanding this relationship will aid in understanding scientific literature as well as bridge disciplines.

\section{Methods}

Ten ( 4 male, 6 female) participants were recruited by word of mouth and by placing fliers about UTHSC. Inclusion criteria were limited to persons 18 to 35 years of age with no history of spine/shoulder/lower limb injuries/defects, and could perform daily activities in the manner of a healthy adult. Pregnant women were also excluded from the study, as determined by a urine pregnancy test. Following radiographs, two female subjects were dropped from the study due to sacralization and lumbarization of vertebrae. The demographics of the remaining eight subjects ( 4 male, 4 female) are provided in Table 2-1.

Table 2-1: $\quad$ Summary of Subject Demographics (Mean \pm SD)

\begin{tabular}{lcccc}
\hline \multicolumn{1}{c}{ Subjects } & Height $(\mathrm{cm})$ & Mass $(\mathrm{kg})$ & BMI $\left(\mathrm{kg} / \mathrm{m}^{2}\right)$ & Age $($ years $)$ \\
\hline Male $(\mathrm{N}=4)$ & $173 \pm 8$ & $76 \pm 7$ & $25.7 \pm 1.3$ & $28 \pm 6$ \\
Female $(\mathrm{N}=4)$ & $168 \pm 4$ & $63 \pm 4$ & $22.1 \pm 0.5$ & $24 \pm 3$ \\
Total $(\mathrm{N}=8)$ & $170 \pm 7$ & $70 \pm 9$ & $23.9 \pm 2.1$ & $173 \pm 8$ \\
\hline
\end{tabular}


To ensure no pregnant women would inadvertently experience radiation during the EOS bi-planar X-ray scans (EOS Imaging, Cambridge, MA, USA), female participants were first required to take a urine pregnancy test administered by a researcher study staff member. No tests suggested pregnancy, and no female subjects were excluded due to this criterion. All study participants wore minimalist clothing (e.g. men: athletic shorts, women: athletic shorts and non-racerbacked sports bra) and underwent a standard physical therapy evaluation by a licensed physical therapist with 25 years of experience. The evaluation consisted of the following: height and weight measurements, the length of both legs as measured from the ASISs to their medial malleoli using a standard tape measure, assessment of joint mobility, muscle strength and flexibility. This evaluation was to screen for any undiagnosed physical limitations.

The same physical therapist then applied thirteen $12.7 \mathrm{~mm}$ retro-reflective markers over the subject's spine and pelvis. The spine markers were adhered to the skin over the C7, T4, T7, T10, T12, L2, L4, S2 spinous processes, as determined by palpation, using double-sided tape and duct tape. Similarly, markers were adhered to the skin over the ASISs and PSISs of the pelvis. The subject would then undergo motion analysis with and without a custom made hyper-tensioned clavicle strap (Figure 2-1). The clavicle strap would be donned and tensioned to the limit of the subject's comfort level. The position of each strap would then be marked and labeled with an indelible ink pen. Following motion analysis, subjects were then escorted to Le Bonheur Children's Hospital where they would undergo two scans from the EOS system.

At Le Bonheur Children's Hospital the subject returned to their minimalist clothing. The subject would then enter the EOS bi-planar X-ray system, and a trained technician would position a radio-translucent handle such that when the subject would
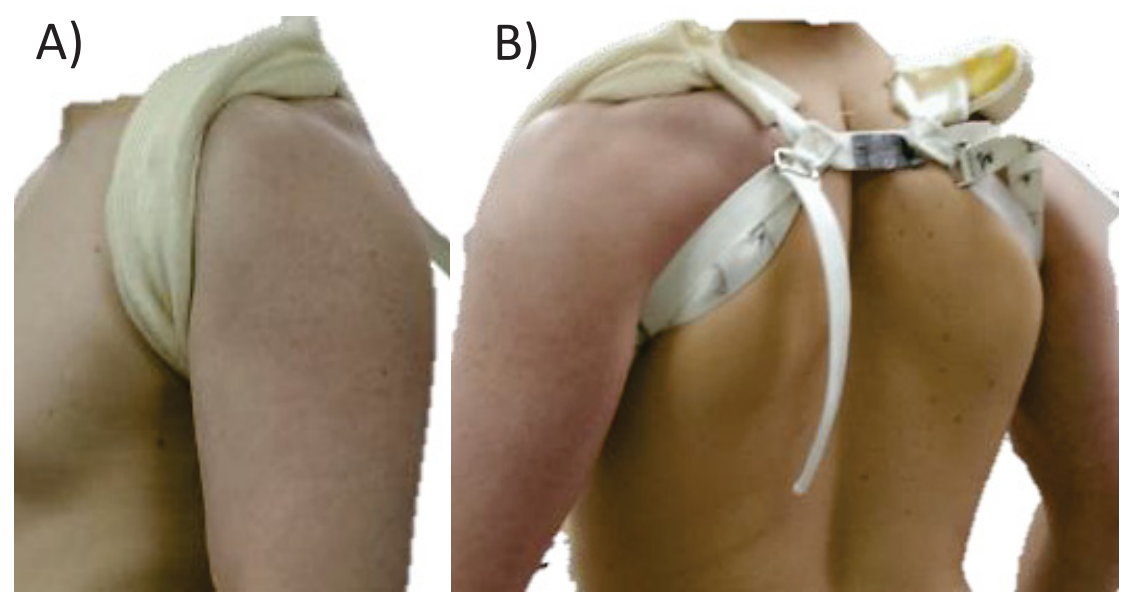

Figure 2-1: Custom Made Clavicle Strap

A) Lateral view of hyper-tensioned clavicle strap B) Oblique view of hyper-tensioned clavicle strap

The clavicle strap was developed with the assistance and guidance of Spears Prosthetics \& Orthotics/ Rehab Services 
lightly hold onto the bar, the subject's humeri would not obscure the lateral project of the spine. The subject was scanned in this position from C7 to mid-thigh. The subject would then don a custom made clavicle strap, and the straps would be tightened to the position marked in indelible ink. With their posture altered they would return to their previous position, with hands held at approximately eye level, and they would be scanned again.

The same two practiced and trained Le Bonheur Children's Hospital technicians analyzed the study subjects. A subject was processed by a single technician. Using the EOS system a technician would generate a 3D reconstruction of the subject's spine and pelvis. If anatomical landmarks were obscured, or in the event of lumbarization or sacralization, the technicians would consult a radiologist or orthopaedic surgeon. From this a report containing LL and TK Cobb angles, as well as radiographic pelvic tilt was generated. The reports, spread sheets, and radiograph images were transferred to UTHSC where differences we calculated using Microsoft Excel 2010 (Microsoft, Redmond, WA). The EOS system defines radiographic pelvic tilt as positive in retroversion and negative in anteversion. This sign convention was changed to be consistent with ASIS-PSIS tilt measures.

The radiographic images were then imported into Fiji, an Open Source image processing package [48], to evaluated marker derived angles as well as APP and anatomical ASIS-PSIS pelvic tilt. Each retro-reflective marker was constructed with a radio-opaque centrally located screw. The center of the flat screw tip was used to approximate the centroid of the marker. By using the screw tips as references it was possible to measure TK and LL as previously used in motion analysis [38, 39]. Fowler et al. (2004) described TK as the acute angle formed by the lines passing the C7 and T4 markers as well as the T10 and T7 markers Figure 1-7C. Similarly, it was possible to measure plumbline [36] and pelvic tilt Figure 1-7B and A. The anatomical locations of the center of C7, ASIS, PSIS, public symphysis, and sacral reference were digitally marked on the radiographs by a PGY-4 orthopaedic resident specializing in spine surgery.

To convert Fiji measures distance measures from pixels to millimeters, the projected area of the markers was used to serve as a means of conversion. The sacrum or L4 marker was used due to their visibility in all radiographs and best approximated the reference position in the sagittal plane. If a marker anatomically left or right of the sacrum was used, the magnification resulting from the dispersion of X-rays would have provided skewed distance measures. By fitting a circle to the marker outline, it was possible to calculate the markers projected area in pixels. From this it was possible to back out the radius of the marker in pixels using the equation for the area of a circle. Knowing the diameter of each marker is $12.7 \mathrm{~mm}$ provided a means to convert pixels to millimeters.

Each measurement was performed three times. If the standard deviation for a set of angle measurements were greater than 0.5 deg all values were deleted and the measures were recalculated. A similar methodology was used for plumbline measures of 
standard deviations greater than $0.5 \mathrm{~mm}$. These thresholds were set to represent the capabilities of the motion analysis system.

\section{Results}

The marker and radiographic measurements for each of the eight remaining subjects are listed in Table 2-1 and Table 2-2. The changes in spine curvature as measured from the markers demonstrated a mean bias of 0 to 3 deg (Table 2-3). For LL, the S2-L4-L2-T12 marker configuration showed smaller differences in the L1/S1 lordosis measure in subjects with a BMI $>25 \mathrm{~kg} / \mathrm{m}^{2}$ while the subjects with a BMI $<25 \mathrm{~kg} / \mathrm{m}^{2}$ showed the smallest differences in the L1/L5 measure (Table 2-1). A Mann-Whitney U Test of the two groups' BMI was found to be statistically significant $(p=0.036)$. The marker based lordosis measure corresponded most (Slope \pm Std. Error) $0.9 \pm 0.3$ deg with the L1/S1 measure when the two groups were not taken into account, but resulted in a mean offset of $3 \mathrm{deg}$. By taking the two groups into account, there resulted an agreement of (mean \pm 2 SD) $1 \pm 6$ deg (Table 2-4). Across all lordosis comparisons increased LL showed more variability than decreased lordosis changes (Figure 2-2A-C).

Both TK and plumbline demonstrated a nearly 1:1 correlation once an offset was applied. However, the marker plumbline measure demonstrated large differences from the clinical plumbline and tends to underestimate plumbline by nearly a centimeter (mean $\pm 2 \mathrm{SD}$ ) $-9 \mathrm{~mm} \pm 25 \mathrm{~mm}$ (Table 2-2). The differences between the two methods is less than $1 \mathrm{~cm}$ in five of the eight subjects, no physical attribute distinguished the other three from the group. The TK markers on average overestimated the amount of change in the thoracic spine by $3 \mathrm{deg}$ and as large as $8 \mathrm{deg}$ (Figure 2-3A). The location of C7, T4, T7, and T10 markers have not been determined at this time and may explain large deviations (e.g. Subject 9)

A Bland-Altman plot revealed that the relationship between pelvic markers and ASIS-PSIS tilt was not 1:1 (Figure 2-3B). Regression analysis revealed that the ASISPSIS tilt to marker based pelvic tilt was nearly 2.5:1. However, the marker pelvic tilt showed a 1:1 relationship with radiographic pelvic tilt once an outlier was removed and an offset was applied (Table 2-3). The marker based pelvic tilt actually showed the smallest span of differences with radiographic pelvic tilt as well as standard error of the estimate (Table 2-3).

What is most note-worthy is that the clinical based pelvic tilt measurements agreed less with each other than with the marker based tilt. When comparing the clinical based measures of pelvic tilt mean differences ranged from -3 to $1 \mathrm{deg}$ and standard deviation of the differences as high as $7 \mathrm{deg}$ (Table 2-3). Once an offset was applied, the radiographic pelvic tilt and ASIS-PSIS tilt showed a $1 \pm 0.3$ deg relationship. The APP showed a poor relationship with either the radiographic pelvic tilt or ASIS-PSIS tilt.

Additional Bland-Altman plots relating clinical and marker measures can be found in Appendix B (Figure B-1 and B-2). 
Table 2-2: $\quad$ Summary of Lumbar Lordosis and Pelvic Tilt Changes Evaluated in EOS X-Ray System

\begin{tabular}{|c|c|c|c|c|c|c|c|c|}
\hline \multirow[b]{2}{*}{ Subject } & \multicolumn{4}{|c|}{ Change in Lumbar Lordosis (deg) } & \multicolumn{4}{|c|}{ Change in Pelvic Tilt Measures (deg) } \\
\hline & $\begin{array}{l}\text { Marker } \\
\text { Lordosis }\end{array}$ & $\begin{array}{c}\text { L1/S1 } \\
\text { Lordosis }\end{array}$ & $\begin{array}{l}\text { L1/L5 } \\
\text { Lordosis }\end{array}$ & Marker Position & $\begin{array}{c}\text { Marker } \\
\text { Tilt }\end{array}$ & $\begin{array}{c}\text { Radiographic } \\
\text { Pelvic Tilt }\end{array}$ & $\begin{array}{c}\text { ASIS- } \\
\text { PSIS Tilt }\end{array}$ & APP \\
\hline $1 *$ & -2 & $\underline{3}$ & 8 & S2,L4,L2,T12 & 2 & 1 & 1 & 0 \\
\hline 2 & -10 & -15 & -10 & S2,L5,L3,L1 & 3 & 0 & - & - \\
\hline $3 *$ & -5 & $\underline{-6}$ & -1 & $\mathrm{~S} 2, \mathrm{~S} 1, \mathrm{~L} 3, \mathrm{~L} 1$ & 1 & 0 & -7 & 9 \\
\hline $5 *$ & 4 & $\underline{2}$ & 1 & S2,L5,L2,T12 & 6 & 11 & 11 & 10 \\
\hline 6 & 8 & 0 & $\underline{2}$ & $\mathrm{~S} 2, L 4, \mathrm{~L} 1, \mathrm{~T} 11$ & 2 & 4 & 1 & 5 \\
\hline 8 & -5 & -4 & $\underline{-5}$ & S1,L4,L2,T12 & 3 & 4 & 4 & -2 \\
\hline 9 & -4 & -5 & $\underline{-4}$ & $\mathrm{~S} 2, \mathrm{~S} 1, \mathrm{~L} 3, \mathrm{~L} 2$ & 1 & -2 & 1 & 8 \\
\hline 10 & -4 & -11 & $\underline{-4}$ & S2,L4,L2,T12 & -1 & -1 & -3 & 1 \\
\hline $\begin{array}{l}\text { Mean } \pm \\
\text { SD }\end{array}$ & $-2 \pm 6$ & $-5 \pm 6$ & $-2 \pm 5$ & & $2 \pm 2$ & $2 \pm 4$ & $1 \pm 6$ & $4 \pm 5$ \\
\hline
\end{tabular}

Notes:

Measures $=$ Orthotic - Normal

- = ASIS was not visible on lateral radiograph.

$\underline{\text { Underline }}=$ Lordosis measurement least different from markers.

Italicized $=$ Marker was closer to L4 spinous process but closer to L3 inferior endplate.

* $27 \mathrm{~kg} / \mathrm{m}^{2}>\mathrm{BMI}>25 \mathrm{~kg} / \mathrm{m}^{2}$

A Mann-Whitney U Test found two BMI groups resulted in $\mathrm{p}=0.036$.

Subjects 4 and 7 were found to have a lumbarized sacral vertebra and a sacralized lumbar vertebra, respectively. 
Table 2-3: $\quad$ Summary of Plumbline and Thoracic Kyphosis Changes

\begin{tabular}{cccccc}
\hline \multirow{2}{*}{ Subject } & \multicolumn{2}{c}{ Change in Plumbline (mm) } & & \multicolumn{2}{c}{ Change in Kyphosis (deg) } \\
\cline { 2 - 3 } \cline { 5 - 6 } & Markers & Clinical & & Markers & T1/T12 Kyphosis \\
\hline $1^{*}$ & 22 & 10 & & 3 & 1 \\
2 & 40 & 70 & & -8 & -9 \\
$3^{*}$ & 13 & 19 & & 4 & -1 \\
$5^{*}$ & 16 & 23 & & 13 & 16 \\
6 & -48 & -39 & & 5 & -3 \\
8 & 18 & 21 & & 1 & 1 \\
9 & -4 & 17 & & -8 & -15 \\
10 & 19 & 27 & & 4 & 4 \\
Mean \pm SD & $10 \pm 26$ & $19 \pm 29$ & & $2 \pm 7$ & $-1 \pm 9$ \\
\hline
\end{tabular}

Notes:

Measures $=$ Orthotic - Normal

$* 27 \mathrm{~kg} / \mathrm{m}^{2}>$ BMI $>25 \mathrm{~kg} / \mathrm{m}^{2}$

A Mann-Whitney U Test found two BMI groups resulted in $\mathrm{p}=0.036$.

Table 2-4: $\quad$ Summary of Bland-Altman and Regressions for Marker vs Radiographic Comparisons

\begin{tabular}{lcccc}
\hline \multicolumn{1}{c}{ Comparison (y vs x) } & $\begin{array}{c}\text { Mean Difference } \\
(\mathrm{x}-\mathrm{y}) \pm 2 \mathrm{SD}\end{array}$ & $\begin{array}{c}\text { Slope } \pm \text { Std. } \\
\text { Error }\end{array}$ & $\begin{array}{c}\text { Intercept } \pm \\
\text { Std. Error }\end{array}$ & S.E.E \\
\hline cPL vs mPL & $-9 \mathrm{~mm} \pm 25 \mathrm{~mm}$ & $1.0 \pm 0.2 \mathrm{~mm}$ & $9 \pm 5 \mathrm{~mm}$ & $13.2 \mathrm{~mm}$ \\
T1/T12 vs mTK & $3 \pm 8^{\circ}$ & $1.2 \pm 0.2^{\circ}$ & $-3 \pm 1^{\circ}$ & $3.8^{\circ}$ \\
L1/S1 vs mLL & $3 \pm 8^{\circ}$ & $0.9 \pm 0.3^{\circ}$ & $-3 \pm 2^{\circ}$ & $4.4^{\circ}$ \\
L1/L5 vs mLL & $0 \pm 9^{\circ}$ & $0.6 \pm 0.3^{\circ}$ & $0 \pm 2^{\circ}$ & $4.3^{\circ}$ \\
RepCobb vs mLL & $1 \pm 6^{\circ}$ & $0.7 \pm 0.2^{\circ}$ & $-1 \pm 1^{\circ}$ & $2.5^{\circ}$ \\
ASIS-PSIS Tilt vs mPT & $1 \pm 8^{\circ}$ & $2.4 \pm 0.8^{\circ}$ & $-4 \pm 2^{\circ}$ & $2.9^{\circ}$ \\
rPT vs mPT & $0 \pm 5^{\circ}$ & $1.8 \pm 0.5^{\circ}$ & $-2 \pm 1^{\circ}$ & $2.4^{\circ}$ \\
** rPT vs mPT & $0 \pm 4^{\circ}$ & $1 \pm 0.7^{\circ}$ & $-1 \pm 1^{\circ}$ & $2.2^{\circ}$ \\
APP vs mPT & $-2 \pm 9^{\circ}$ & $0.7 \pm 1.0^{\circ}$ & $3 \pm 3^{\circ}$ & $5.0^{\circ}$ \\
APP vs ASIS-PSIS Tilt & $-3 \pm 14^{\circ}$ & $0.1 \pm 0.4^{\circ}$ & $4 \pm 2^{\circ}$ & $5.2^{\circ}$ \\
ASIS-PSIS Tilt vs rPT & $1 \pm 6^{\circ}$ & $1 \pm 0.3^{\circ}$ & $1 \pm 2^{\circ}$ & $3.6^{\circ}$ \\
APP vs rPT & $-2 \pm 12^{\circ}$ & $0.2 \pm 0.5^{\circ}$ & $-4 \pm 2^{\circ}$ & $5.1^{\circ}$ \\
\hline
\end{tabular}

Notes:

$\mathrm{c}=$ Clinical, $\mathrm{m}=$ Marker, $\mathrm{r}=$ Radiographic. RepCobb $=$ Representative Cobb Angle, PL

$=$ Plumbline, $\mathrm{TK}=$ Thoracic Kyphosis, $\mathrm{LL}=$ Lumbar Lordosis, ASIS-PSIS $=$ ASIS-PSIS

Tilt

rPT was defined with anteversion as positive

** Values were calculated without outlier. 




Figure 2-2: Bland-Altman Plots of Marker vs EOS Derived Lumbar Lordosis *The Cobb angle measure with the smallest difference from the marker measure was used.

Solid black line represents mean difference between measurements Dashed lines represent the mean $\pm 2 * \mathrm{SD}$ 


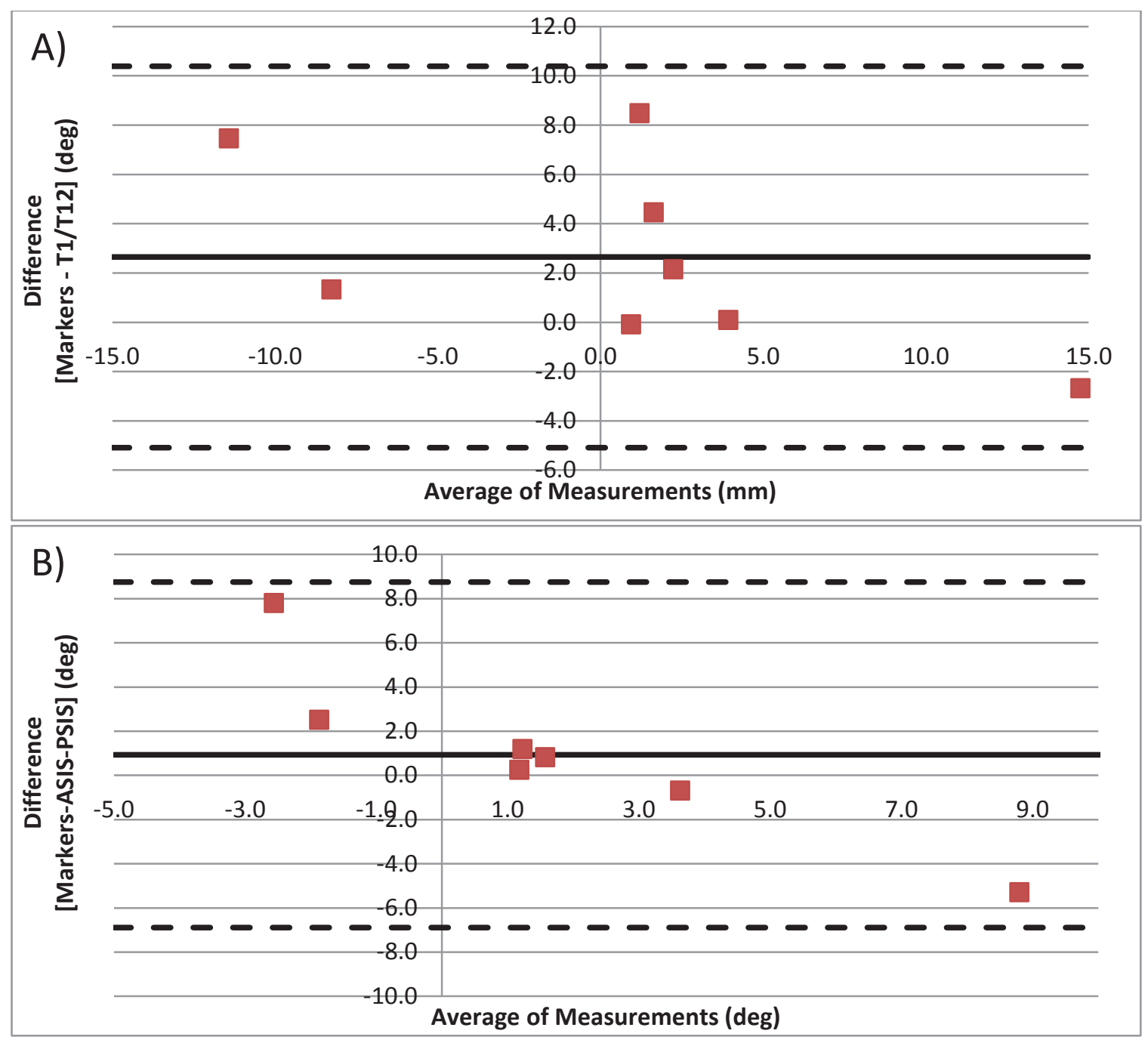

Figure 2-3: Marker vs Clinical Bland-Altman Plots for Thoracic Kyphosis and Pelvic Tilt

Solid black line represents mean difference between measurements

Dashed lines represent the mean $\pm 2 * \mathrm{SD}$ 


\section{Limitations and Assumptions}

It is important to interpret the results of this study with consideration to the study's limitations and assumptions. The study consisted of eight health young adults, with BMIs range from 21.1 to $26.9 \mathrm{~kg} / \mathrm{m}^{2}$. Subjects were evaluated in static standing posture with generally less than $10 \mathrm{deg}$ of change. Another limitation was the palpated location of skin markers were not consistently placed over the intended vertebrae; a limitation common in opto-electronic studies. It was assumed: that using the screw heads in the markers accurately reflected an opto-electronic camera system, and that the EOS system has an accuracy equal to or better than the accuracy of skin markers $( \pm 0.5 \mathrm{deg})$ [33].

\section{Discussion}

There was a broad range of agreement between marker and clinical measures in this study. The marker measures for some subjects (e.g. Subject 10, Table 2-1) showed a perfect agreement in L1/L5 LL, TK, and pelvic tilt; while others (e.g. Subject 6) showed a very poor agreement. In Subject 6's case, this was probably due to poor marker position with each marker shifted superiorly approximately 1 vertebra (Table 2-1). Similarly, subjects 1, 3, and 5 showed large deviations in L1/L5 LL. These subjects were found to be statistically different $(\mathrm{p}=0.036)$ from the group when BMI. Their BMI being greater than $25 \mathrm{~kg} / \mathrm{m}^{2}$ and may explain their lack of agreement with clinical measures. If these four subjects are no longer considered, the marker agreement with L1/L5 becomes (mean $\pm 2 \mathrm{SD}$ ) $0 \pm 1 \mathrm{deg}$. However, the agreement is based upon only 4 subjects, and high BMI as well as imprecise marker placement are persistent challenges in motion analysis and cannot be ignored. Overall, the angular measures agree within approximately $10 \mathrm{deg}$ of radiographic measures and have the potential to become much more precise if challenges with BMI and marker placement can be addressed.

One surprising finding was the amount of deviation between marker and clinical plumbline. The $\mathrm{C} 7$ spinal process is very prominent on normal BMI individuals, easily palpated, and this comparison is looking at changes in anterior displacement. It is difficult to believe that the clinical plumbline can shift $3 \mathrm{~cm}$ more anteriorly than the marker measure (Table 2-2). The most likely causes of this discrepancy are due to the subjectivity of marking the clinical plumbline in radiographs, the ability of the subject to rotate their pelvis in the transverse plane independently of the torso, and skin folds of the back. As a result, the plumbline measure for this study may under predict changes in clinical plumbline by $9 \mathrm{~mm}$.

An interesting finding in validating the different clinical measures was that marker based pelvic tilt agrees much more with changes in radiographic pelvic tilt than $\mathrm{APP}$, (mean $\pm 2 \mathrm{SD}) 0 \pm 5 \mathrm{deg}$ and $-2 \pm 12$ respectively. This finding suggests that changes in marker based pelvic tilt can predict changes in radiographic pelvic tilt to within $5 \mathrm{deg}$. This is smaller than the deviations found in normative datasets [27, 32]. However, these findings are based on a small sample size with change in radiographic 
pelvic tilt exceed 4 degrees only once. The discrepancy in APP may be attributed to rotations of the pelvis in the transverse plane, but the finding highlights the problem of jumping between the two measurement techniques. The APP-radiographic pelvic tilt relation is especially concerning, given the prevalence of individuals with hip and spine pathologies.

If these different measures are taken at face value the interpretation of the different measures can become skewed or incorrect all together. In order for pelvic and spine information to be understood across a variety of fields it is essential to further understand the various pelvic and spine measures.

To further establish the relationship between different spinal and pelvic tilt measures, future should include a larger sample size, as well as varying ethnicities. Morphological differences alone can vary pelvic tilt as much as 23 deg if comparing the ASIS-PSIS tilt to the anterior pelvic plane [29]. It may be possible to further establish these relationships during dynamic activities with fluoroscopy, or bone pins, and a camera system. 


\section{CHAPTER 3. EOS BI-PLANAR X-RAY VALIDATES HYPER-TENSIONED CLAVICLE STRAP ALTERS LUMBAR LORDOSIS IN STANDING}

\section{Chapter Overview}

Following spine fusion surgery a patient's spine may be fused in an abnormal posture that may result in fixed sagittal imbalance or flatback deformity. While spinopelvic parameters have been thoroughly investigated in static standing, there is a dearth of information when it comes to dynamic activities of daily living. The limited understanding of the spino-pelvic relationship may be attributed to cofounding variables, such as coexisting hip pathologies, or the difficulty in altering spinal position in normal healthy subjects while maintaining visibility of the spine. This study sought to validate the use of a hyper-tensioned clavicle strap to alter lumbar position, and to determine the relationship between changes in sagittal spinal parameters with changes in pelvic tilt during static standing. We hypothesized that a subject would compensate an altered lumbar lordosis by altering their pelvic tilt and torso alignment to maintain a normal plumbline. This study utilized eight young, healthy subjects with no history of joint pain, or surgeries. The subjects were evaluated in a paired fashion using an EOS X-ray system in both a normal and posture altered condition. This validation showed that the hypertensioned clavicle strap was able to alter clinical plumbline, T1/T12 thoracic kyphosis, L1/S1 lumbar lordosis, and radiographic pelvic tilt (mean \pm SD) $1.9 \pm 2.9 \mathrm{~cm},-1 \pm 9 \mathrm{deg}$, $-5 \pm 6 \mathrm{deg}$, and $-2 \pm 4 \mathrm{deg}$, respectively. Clinical plumbline, TK, and LL were linearly correlated to radiographic pelvic tilt, and a Student's-t test was used to determine significance of their respective slopes. Only changes in TK were significantly related to changes in radiographic pelvic tilt $(\mathrm{p}=0.021)$.

\section{Background}

Following SFS a patient's spine may be fused in an abnormal posture [2-13] that may result in FSI or flatback deformity (Figure 1-1). The prevalence of pre- and postoperative radiographs, as well as the ease of collecting static information in the laboratory has allowed for a thorough study of the lumbar spine and pelvis of FSI and flatback patients in the standing position [2-7, 9-14, 16, 19, 25-28, 31, 49]. Individuals with FSI and flatback deformities exhibit an increased plumbline, anterior pelvic tilt, flexion of the lower extremities and decrease in LL $[2,5,6,8,19]$. Due to the complex nature of SFS, as well as the subject's ability to compensate, there are no standard values for FSI or flatback; values of plumbline and LL found in scientific literature can span 5 to $30 \mathrm{~cm}$ and -36 to 33 deg, respectively $[3,8]$. When compared to the range of normative values (Appendix C, Figure C-1) for the same parameters -1.8 to $8.1 \mathrm{~cm}$ and 16 to $89 \mathrm{deg}$ [27], there is significant overlap. As a result, even if the spine surgeon restores LL to a normal range, the subject may still exhibit FSI.

Another challenge regarding FSI and flatback deformities is that evidence

suggests static changes in the spine and pelvis due not carry over to dynamic activities 
[24, 36, 39]. Syczewska et al (1999) showed skin marker based spine angle measures evaluated during walking, increase as much as 6 degrees from static trails [39]. Gottipati et al (2014) showed skin marker measures of plumbline can change more than $11 \mathrm{~cm}$ from static measures [36]. Lee et al (2001) showed even when normal lordosis is restored postoperatively, subjects may still experience a stooped posture during gait [24].

The studies that investigated the dynamic effects of FSI and flatback deformity [8, $24,25,36]$ did not explore the spino-pelvic relationship, and the studies that have investigated the spino-pelvis relationship did not restrict or altered spinal motion [50, 51]. As a result there is little to no literature on how changes in spinal parameters directly relate to changes in pelvic position.

The dearth of information regarding the influence changes in spinal position have on pelvic position may be attributed to challenges in altering spine position, or the prevalence of coexisting pathologies $[15,16]$; as can be the case in subjects with FSI or flatback deformity. If a device, such as a clavicle strap, could be used to induce a change in LL in healthy subjects. It may be possible to better understand how changes in spinal parameters, such as LL, correspond to changes in pelvic tilt during activities of daily living. Ultimately, we believe understanding the spino-pelvic relationship could aid in surgical planning and could reduce the incidence of FSI, and flatback deformity.

The purpose of this study was to validate the use of a hyper-tensioned clavicle strap to alter LL, and to determine the relationship between changes in sagittal spinal parameters with changes in pelvic tilt during static standing. We hypothesized that a subject would compensate an altered lumbar lordosis by altering their pelvic tilt and torso alignment to maintain a normal plumbline.

\section{Methods}

Ten ( 4 male, 6 female) participants were recruited by word of mouth and by placing fliers about UTHSC. Inclusion criteria were limited to persons 18 to 35 years of age with no history of spine/shoulder/lower limb injuries/defects, and could perform daily activities in the manner of a healthy adult. Pregnant women were also excluded from the study. Following radiographs, two female subjects were dropped from the study due to sacralization and lumbarization of vertebrae. The demographics of the remaining eight subjects (4 male, 4 female) are provided in Table 2-1.

To ensure no pregnant women would inadvertently experience radiation during the EOS bi-planar X-ray scans (EOS Imaging, Cambridge, MA, USA), female participants were first required to take a urine pregnancy test administered by a researcher study staff member. No tests suggested pregnancy, and no female subjects were excluded due to this criterion. All study participants wore minimalist clothing (e.g. men: athletic shorts, women: athletic shorts and non-racerbacked sports bra) and underwent a standard physical therapy evaluation by a licensed physical therapist with 25 years of experience. The evaluation consisted of the following: height and weight 
measurements, the length of both legs as measured from the ASISs to their medial malleoli using a standard tape measure, assessment of joint mobility, muscle strength and flexibility. This evaluation was to screen for any undiagnosed physical limitations.

The clavicle strap would then be donned (Figure 2-1) and tensioned to the limit of the subject's comfort level. The position of each strap would then be marked and labeled with an indelible ink pen. Following motion analysis, subjects were then escorted to Le Bonheur Children's Hospital where they would undergo two scans from the EOS system.

At Le Bonheur Children's Hospital the subject returned to their minimalist clothing. The subject would then enter the EOS bi-planar X-ray system, and a trained technician would position a radio-translucent handle such that when the subject would lightly hold onto the bar, the subject's humeri would not obscure the lateral project of the spine. The subject was scanned in this position from $\mathrm{C} 7$ to mid-thigh. The subject would then don the custom made clavicle strap, and the straps would be tightened to the position marked in indelible ink. With their posture altered they would return to their previous position, with hands held at approximately eye level, and they would be scanned again.

The same two practiced and trained Le Bonheur Children's Hospital technicians analyzed the study subjects. A subject was processed by a single technician. Using the EOS system a technician would generate a 3D reconstruction of the subject's spine and pelvis. If bony landmarks were obscured, or lumbarization or sacralization was suspected, the technicians would consult a radiologist or orthopaedic surgeon for a second opinion. From the 3D reconstruction a report containing LL and TK Cobb angles, as well as radiographic pelvic tilt was generated. The reports, spread sheets, and radiographic images were transferred to UTHSC where differences we calculated using Microsoft Excel 2010 (Microsoft, Redmond, WA). The EOS system defines radiographic pelvic tilt as positive in retroversion and negative in anteversion. This sign convention was changed to be consistent with ASIS-PSIS tilt measures.

The radiographic images were then imported into Fiji, an Open Source image processing package [48], to evaluate clinical plumbline. The anatomical locations of the center of C7 sacral reference were digitally marked on the radiographs by a PGY-4 orthopaedic resident specializing in spine surgery. To convert clinical plumbline measures form pixels to millimeters, a $12.7 \mathrm{~mm}$ retro-reflective marker was attached to the skin over the subject's sacrum and L4 spinous process prior to EOS scans. These marker locations best approximated the sacral reference depth in the coronal plane. The marker used depended on visibility in the lateral radiographs. By fitting a circle to the marker outline, it was possible to calculate the markers projected area in pixels. From this it was possible to back out the radius of the marker in pixels using the equation for the area of a circle. Knowing the diameter of each marker is $12.7 \mathrm{~mm}$ provided a means to convert pixels to millimeters. If a marker anatomically left or right of the sacrum were to be used, the magnification resulting from the dispersion of X-rays would have provided skewed distance measures. 
Clinical plumbline was measured three times for each subject. If the standard deviation for a set of measurements were greater than $0.5 \mathrm{~mm}$ all values were deleted and the measures were recalculated. This threshold was set to represent the capabilities of a motion analysis system.

\section{Results}

The changes in L1/S1 LL and radiographic pelvic tilt ranged from 3 to $-15 \mathrm{deg}$ and 2 to $-11 \mathrm{deg}$ of change, respectively (Figure 3-1). The changes in plumbline and T1/T12 TK ranged from -39 to $70 \mathrm{~mm}$, and -15 to $16 \mathrm{deg}$, respectively (Figure 3-1). Considering the wide range of values it is no surprise a wide range of compensations occurred (Figure 3-1). Despite what has been reported in previous literature, displacement of the plumbline or alterations in lordosis did not significantly correlate with changes in pelvic tilt $(\mathrm{p}>0.6$ and $\mathrm{p}>0.1$, respectively). There was a significant correlation between changes in T1/T12 TK and changes in radiographic pelvic tilt $(\mathrm{p}=0.021)$ (Table 3-1, and Figure B-3).

\section{Limitations and Assumptions}

It is important to interpret the results of this study with consideration to the study's limitations and assumptions. The study was limited to eight healthy, young adults, with BMIs from 21.1 to $26.9 \mathrm{~kg} / \mathrm{m}^{2}$. The hyper-tensioned clavicle strap was not tensioned empirically (e.g. to 30lb), and changes in lordosis were subject specific. To limit radiation exposure, subjects were limited to only two EOS scans. During the EOS scans the subject's arm position was altered from a normal standing position, and their foot position was controlled. It was assumed: that the hyper-tensioned clavicle strap was tightened equally on both sides, and that the EOS system had an accuracy equal to or better than the accuracy of skin markers $( \pm 0.5 \mathrm{deg})[33]$.

\section{Discussion}

It is believed by some that the end goal of a standing posture is to bring the head upright so the individual can see what is in front of them [2]. In flatback deformities the LL is flattened, and the modes in which subjects compensate for this depends on the capabilities of their body.

On average the subjects in this study demonstrated a hyper-tensioned clavicle strap could clinical plumbline, T1/T12 TK, L1/S1 LL, and radiographic pelvic tilt -3.9 to $7.0 \mathrm{~cm}$, and -15 to $16 \mathrm{deg}, 3$ to -15 and 2 to $-11 \mathrm{deg}$ respectively. Some small increases in LL were seen, the majority of individuals demonstrated a decreased LL by more than 4 degs. These finds suggest that a hyper-tensioned clavicle strap is capable of altering LL. However, further studies, with a larger sample population and multiple static trials would be needed to determine the degree to which a hyper-tensioned clavicle strap can alter LL. 


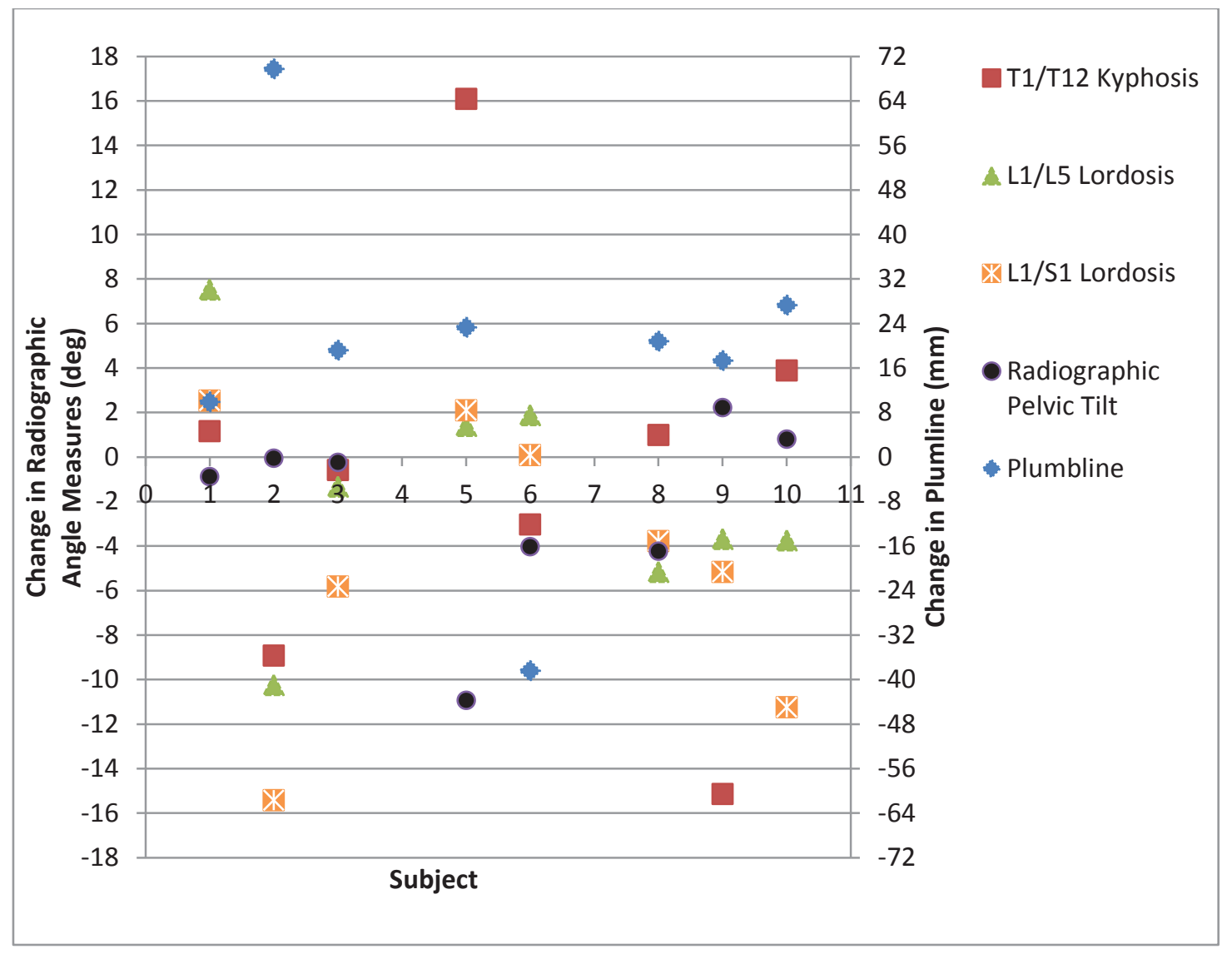

Figure 3-1: Individual Subject Responses to Orthotic

Table 3-1: $\quad$ Summary of Radiographic Correlations

\begin{tabular}{lccccc}
\hline \multicolumn{1}{c}{$\mathrm{N}=8$} & $\mathrm{R}^{2}$ & Adjusted $\mathrm{R}^{2}$ & S.E.E & *Slope \pm Std. Error & $\neq \mathrm{P}-$ Value \\
\hline rPT vs cPL & 0.043 & -0.117 & 4.4 & $0.03 \pm 0.06$ & 0.622 \\
rPT vs T1/T12 & 0.614 & 0.55 & 2.8 & $-0.4 \pm 0.1$ & $\mathbf{0 . 0 2 1}$ \\
rPT vs L1/L5 & 0.089 & -0.062 & 4.3 & $-0.2 \pm 0.3$ & 0.472 \\
rPT vs L1/S1 & 0.334 & 0.223 & 3.7 & $-0.4 \pm 0.2$ & 0.133 \\
\hline
\end{tabular}

Notes:

$\mathrm{rPT}=$ Radiographic Pelvic Tilt, $\mathrm{cPL}=$ Clinical Plumbline, T1/T12= T1/T12 Cobb Angle, L1/L5= L1/L5 Cobb Angle, L1/S1= L1/S1 Cobb Angle, S.E.E = Standard Error of Estimate

* Slopes and Standard Errors are for unstandardized coefficients

$\ddagger \mathrm{P}$-Values were calculated using a Student's- $-\mathrm{t}$ test of the regression slope. 
The relationship between clinical plumbline, TK, LL, and radiographic pelvic tilt have been fairly well established in literature [2, 3, 5, 6, 8, 12, 14, 24, 27], was not seen in this study. No significant correlations with pelvic tilt were demonstrated with LL or clinical plumbline. The remaining parameter, T1/T12 TK, showed a significant $(p=0.021)$ nearly $-2: 1$ relationship with radiographic pelvic tilt. It is import to keep in mind these correlations were defined by 8 subjects, and changes in kyphosis spanned $30 \mathrm{deg}$. By looking at Figure 3-1 it becomes apparent why a correlation could not be established. The degree of changes and degrees of compensation are wide spread. For example, it appears subject 2 made no effort to correct their plumbline. Both their thoracic and lumbar spine was flattened and their pelvic tilt remains unchanged. Then consider subject 5, who underwent a $16 \mathrm{deg}$ increase in TK and compensated primarily in the pelvis.

Another possible explanation for this uncharacteristic compensation pattern includes the testing conditions themselves. The subjects are placed in a restrictive brace, in a small confined space, standing in an area approximately 4 feet square, with their foot position controlled. They are then asked to place their hands on a bar at eye level. Even though the subjects were instructed not to strain themselves, and that they should be relaxed, these conditions may encourage the subject to stand in an erect posture instead of acquiring a relaxed posture. Another study that used the same EOS x-ray technology, investigated the lumbar-pelvis relationship [14], and made no comments on the physical restrictions of the system. However, this study was unique in that an external orthosis was attached which restricted arm motion as well as thoracic curvature. 


\section{CHAPTER 4. SPINO-PELVIC KINEMATICS OF GAIT UNDER DIFFERENT SAGITTAL ALIGNMENTS}

\section{Chapter Overview}

Altering the position and motion of the lumbar spine can significantly impact a person's ability to ambulate. Fixed sagittal imbalance (FSI) or flatback deformities can cascade to alter the position and motion of the hips, knees, and ankles, resulting in a crouched and inefficient gait. No prior study has sought to understand the amount an altered spine posture is compensated during the gait. This study utilized ten young, healthy subjects with no history of spine or low extremity pathologies or surgeries. The subjects were evaluated in a paired fashion performing ambulation in a ten camera motion analysis laboratory. A custom hyper-tensioned clavicle strap was used to alter spine curvature. Intrasubject changes in lumbar lordosis (LL), pelvic tilt, plumbline, hip flexion, and flexion of the trunk relative to the pelvic (Trunk-Pelvic angle) were analyzed throughout the whole gait cycle. A Wilcoxon Signed-Rank Test was used to determine significance of changes, and a Student's-t test was used to determine significance of the linear regression slopes. This study was able to decrease LL (mean $\pm \mathrm{SD}$ ) $-4 \pm 2$ deg $(\mathrm{p}=0.008)$, while there was little to no change in anterior pelvic tilt $1 \pm 1 \mathrm{deg}$. However, two distinct modes of compensation were present $(p<0.0001)$. Three of the 9 subjects presented with clinically tight hamstring (popliteal-angle $<-25$ degs), and exhibited an approximately 2:1 change in LL to change in pelvic tilt. The remaining 6 showed essentially no LL to pelvic tilt relationship, (slope \pm std. error) $0.06 \pm 0.04$.

\section{Background}

Altering the position and motion of the lumbar spine can significantly impact a person's ability to ambulate. In the case of FSI and flatback deformity, the gross implications are well understood $[8,24,25,36]$. Lee et al (2001) examined the influence of sagittal imbalance in subjects with flatback deformities [24]. They found that subjects who exhibited postoperative stooping had a markedly anterior pelvic tilt compared to their counterparts [24]. This finding suggests the lumbar-pelvic relationship significantly affects functional performance. Sarwahi et al (2002) found patients who had SFS resulting in flatback deformity exhibited significantly decreased spatiotemporal parameters during gait including velocity, step length, and cadence when compared to normal controls [8]. This trend was confirmed by Oken et al (2011) who examined the gait of patients with varying numbers of disks fused during SFS [25]. The effects of crouched gait resulting from FSI or flatback, as described by increased torso, hip, knee, and ankle flexion, on energy consumption, and joint health has predominately been limited to subjects with cerebral palsy [52]. These studies show crouched gait increases energy expenditure, as measured by oxygen-uptake and heart-rate [52], as well as joint pain and degeneration [53]. However, there is evidence to suggest that these characteristics are prevalent in subjects with FSI [15, 19]. The aforementioned gait 
studies, when considering the spine, considered the gross spine and did not compare changes in lumbar spine position to pelvic position.

While gross trunk motion has been well reported during gait, lumbar and thoracic motion is still minimally investigated $[35,37-39,54,55]$. It has been shown by Needham et al (2014) that lumbar-pelvic coordination in the sagittal plane occurs primarily inphase and with the lumbar preceding the pelvis [55]. This study adds to the theory that the complex motion of the spine acts as a motor for human gait [56]. Despite the importance of the spine during gait, little is known about the compensation mechanisms when the curvature and motion of the spine is changed or diminished, as can occur after SFS.

The purpose of this study was to determine how changes in spinal position correspond with changes in pelvic tilt during gait, utilizing a custom hyper-tensioned clavicle strap. Preliminary investigations in our lab suggested that a hyper-tensioned clavicle strap would be able to consistently alter LL during gait, without obscuring the spinous processes or purchasing on the pelvis (Appendix C). Similarly, a radiographic validation study was conducted to ensure, that the hyper-tensioned clavicle strap alters LL (Chapter 3). Both the preliminary testing and validation study suggested that the clavicle strap could alter LL. We hypothesized that a subject would compensate an altered lumbar lordosis by altering their pelvic tilt and torso alignment to maintain a normal plumbline.

\section{Methods}

\section{Participants}

Participants were recruited by word of mouth and by placing fliers about the UTHSC campus. Inclusion criteria were limited to persons 18 to 35 years of age with no history of spine/shoulder/lower limb injuries/defects, and could perform daily activities in the manner of a healthy adult. Pregnant women were also excluded from the study. Ten subjects were incorporated in this study (4 male, 6 female). However, one subject was determined to be an outlier and was not included in the analysis. A table containing the demographics of the remaining population is provided in Table 4-1.

\section{Procedures}

Each subject was brought to the Motion Analysis Lab as part of the UTHSC, Department of Physical Therapy. The instruments used in the Motion Analysis Lab consisted of ten opto-electronic cameras (Qualisys AB, Gothenburg, Sweden), and 3 force plates (AMTI, Watertown, MA, USA). The cameras were calibrated to an accuracy of $< \pm 0.6 \mathrm{~mm}$ (mean $= \pm 0.4 \mathrm{~mm}$ ) of the calibration wand length, and the force plates were zeroed prior to each test. 
Table 4-1: $\quad$ Summary of Subject Demographics (Mean \pm SD)

\begin{tabular}{lcccc}
\hline \multicolumn{1}{c}{ Subjects } & Height $(\mathrm{cm})$ & Mass $(\mathrm{kg})$ & BMI $\left(\mathrm{kg} / \mathrm{m}^{2}\right)$ & Age $($ years $)$ \\
\hline Male $(\mathrm{N}=4)$ & $173 \pm 8$ & $76 \pm 7$ & $25.7 \pm 1.3$ & $28 \pm 6$ \\
Female $(\mathrm{N}=5)$ & $167 \pm 4$ & $62 \pm 3$ & $18.5 \pm 1.0$ & $23 \pm 3$ \\
Total $(\mathrm{N}=9)$ & $170 \pm 7$ & $69 \pm 9$ & $22.1 \pm 2.1$ & $26 \pm 5$ \\
\hline
\end{tabular}

Before retro-reflective markers were applied, the subjects would change into minimalist clothing (e.g. men: athletic shorts, women: athletic shorts and nonracerbacked sports bra), and a licensed physical therapist with 25 years experience would perform a standard physical therapy evaluation which included the following: height and weight measurements, the length of both legs as measured from the ASISs to their medial malleoli using a standard tape measure, assessment of joint mobility, muscle strength and flexibility. This evaluation was to screen for any undiagnosed physical limitations.

The same physical therapist then applied thirty-two $12.7 \mathrm{~mm}$ reflective markers over anatomical landmarks of the torso and bilaterally over the lower extremities Figure 4-1A. All subjects had 9 markers placed on their torso: left and right acromion (ACR), and spinous processes $\mathrm{C} 7, \mathrm{~T} 4, \mathrm{~T} 7, \mathrm{~T} 10, \mathrm{~T} 12, \mathrm{~L} 2, \mathrm{~L} 4 ; 4$ markers defined the pelvis: right and left ASISs and PSISs; 3 markers over the sacrum (SACR): one over S2, and two inferiorly (SL, SR); a total of 16 markers were placed bilaterally over the medial and lateral epicondyles (MED \& LATFEMCON) and malleoli (MED \& LATMALL), calcaneus (CALC), dorsum (DRSM), $5^{\text {th }}$ metatarsal head $(5 \mathrm{TH})$, as well as the great toe (GTOE) were used to define the lower extremities. Clusters of 4 markers secured to a plastic base were attached laterally to the thighs and shanks using self-adhering elastic wraps to aid in tracking segment motion. Additional $9.5 \mathrm{~mm}$ markers were added in between marked processes when possible (F\#). These markers served to further define the curvature of the full spine as well as to mark lumbar and thoracic apexes. All markers were attached using double-sided tape, and clothing was taped such that the fabric would not obscure markers. Duct tape was added to spinal and pelvic markers, to ensure their fixation.

After the markers were attached the barefoot subject was positioned, in the middle of a specially designed 25 foot-long platform instrumented with three force plates, with each foot on a force plate. The subject was instructed to stand in a normal relaxed position with their hands held in front of their face, as if they were doing a pull-up, and elbows brought anteriorly away from their torso. This position was used because it allowed full visibility of markers and served as part of a separate study. The subject was recorded twice for one second in the standing position. These static captures are used to generate a 6 degree of freedom (DOF) linked segment model.

Medial markers were then removed, and the subject was instructed to walk back and forth along the platform. After several practice trials to allow the subject to adjust to the new environment and attachments, the subject was instructed to walk across the platform at a comfortable self-selected walking speed. The subject was recorded for 


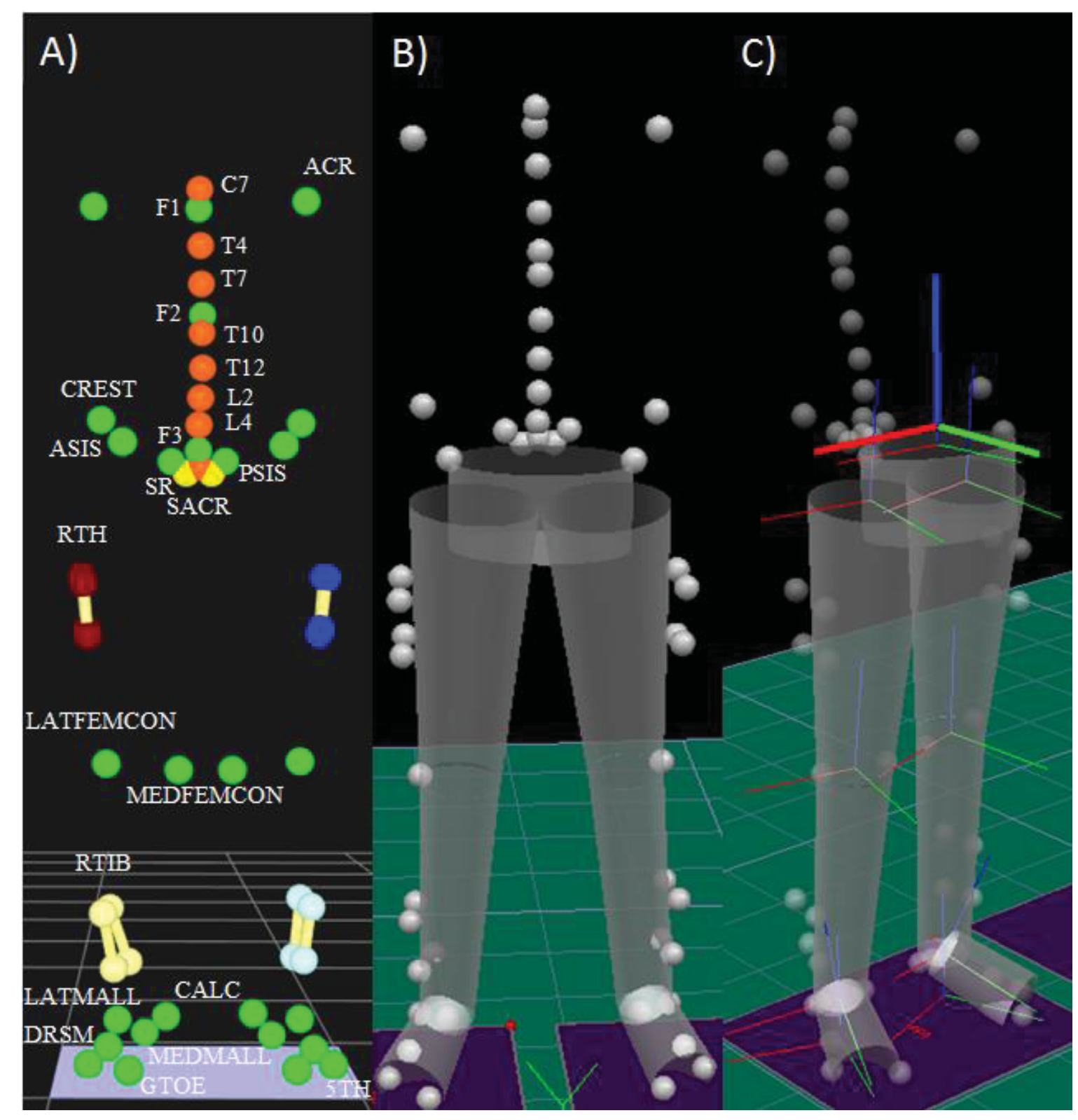

Figure 4-1: $\quad$ Testing Markerset and Geometric Model

A) Markerset B) Geometric model C) Segment coordinate systems. Bolded axes located at ASIS level represent transverse, sagittal, and coronal axes 
approximately 10 (5 left, 5 right) successful trials. A trial was considered successful when a foot cleanly hit the force plate without any portion of the foot touching or crossing the edge of the force plate.

With the normal session completed, the subject was equipped with a modified clavicle strap. The strap was designed and manufactured with the aid of Spears Orthosis and Prosthetics to hold the shoulders back with minimal obstruction of the spinous processes (Figure 2-1). The straps were tightened to the limit of the subject's comfort level, and the position of each strap was marked using an indelible ink pen. If the clavicle strap obscured the C7 or T4 marker, it was rotated about the shoulders until the marker was no longer obscured. However, due to skin folds and sports bra straps some thoracic measurements were lost.

The subject was then instructed to walk along the platform to allow them to become familiar with the device, and altered posture. Once the subject was comfortable with the new posture, they were again brought to the middle of the capture volume and two static measurements were collected. The subject would then repeat the gait activity in the same manner as previously described.

\section{Kinematic Analysis}

All activities were recorded at $100 \mathrm{~Hz}$ using ten opto-electronic cameras (Qualisys $\mathrm{AB}$, Gothenburg, Sweden), marker position was interpolated over a maximum of 10 frames using a $3^{\text {rd }}$ order polynomial, and low pass filled at $7 \mathrm{~Hz}$ using a $4^{\text {th }}$ order digital Butterworth filter. Marker position of static trials was processed in the same manner at $2 \mathrm{~Hz}$. Ground reaction forces and center of pressures were low pass filtered at $15 \mathrm{~Hz}$. Kinematics and ground reaction forces were recorded using Qualysis 8.9 and then exported to Visual3D version 5 for processing.

In Visual3D the first normal static trial of each subject was used to generate a 6 DOF rigid-linked segment model composed of 7 simple geometric shapes similar to those described by Hanavan (1964). The pelvis and torso were modeled as right elliptical cylinders, while the foot, shank, and thigh of each leg were model as frustra of right cones as illustrated in Figure 4-1B. The radius of the proximal superior end of the femur was calculated to be half the distance between each hip joint. The hip joints were approximated by Equation 1-2. The midpoints of the malleoli and epicondyles of each leg represented the ankle and knee joints, respectively. The same markers that defined the geometric shapes then defined the local segment axes (Figure 4-1C).

The sagittal plumbline, as measured by the anterior displacement of the C7 marker from the S2 sacrum marker, TK, LL, and pelvic tilt were calculated in the sagittal plane. The sagittal plane was defined as the vertical plane (with respect to gravity) which passed through the midpoints of the PSISs and ASISs. The flexion of the hips was calculated by the rotation of the femur segment about the mediolaterial axis of the pelvis. 
Each successful gait cycle, heel-strike (HS) to successive ipsilateral heel-strike, was segregated into left and right gait cycles (LHS-to-LHS and RHS-to-RHS) and normalized to $100 \%$. HS was define as when the center of gravity of the foot segment fell within $0.2 \mathrm{~m}$ of the force plates center of pressure, and the resultant force exceeded $20 \mathrm{~N}$. If the successive ispilateral heel strike did not land on a force plate a pattern recognition algorithm, as described by Stanhope et al (1990), was used. If the algorithm [57] did not have enough information to be applied correctly, the minimum vertical height of the CALC marker was used. The minimum vertical displacement method and pattern recognition method agreed well when applied concurrently.

The normalized left and right gait cycles in the normal condition, minimum of 5 each, were averaged to create mean curves. The difference in LL, plumbline, pelvic tilt, $\mathrm{TK}$, and hip flexion of each normalized orthotic trial from the corresponding mean curve was then calculated in 1\% increments and arithmetically averaged. The three orthotic gait cycles with the greatest average decrease in LL and the three orthotic gait cycles with the greatest average LL increase (total 6), for the left and right gait cycles was determined. The side (e.g. left) with the greatest lordosis change was exported to Microsoft Excel 2010 (Microsoft, Redmond, WA) where the aforementioned parameters were arithmetically averaged over the three trials. If an orthotic cycle did not contain all parameters of interest, excluding TK, a trial nearest in magnitude and same direction of LL change was used. TK was excluded from this criterion due to the orthosis and bra straps obscuring markers. If LL changed in less than three cycles in the same direction the cycles were neglected.

\section{Statistical Analysis}

Prior to statistical analysis, UTHSC biostatistician Jim Wan was consulted to determine the most appropriate methods for our variables in question.

A Wilcoxon signed-rank tests with a Holms-Sidak correction was used to determine if the parameters of the orthotic condition were statistically different from those of the normal condition. Linear regression analysis was then performed comparing changings in LL, plumbline, and trunk-pelvic angle to changes in pelvic tilt and hip flexion. The slopes of each linear regression were then compared to zero using a Student's-t test. IBM SPSS Statistics 21 program (IBM Corporation, Armonk, NY) was used to perform Wilcoxon signed-rank tests, linear regression analysis, and Student's-t tests. Sub-groups were compared using Equations 4-1 and 4-2 when at least one group was found to have a statistically significant correlation. $S^{2}$ pool is the pooled estimate of the variance around the two regression lines, and $S^{2}{ }_{c o m}$ is the estimated variance of a

$$
\begin{gathered}
S^{2}{ }_{i m p}=\frac{\left(n_{1}+n_{2}-2\right) * S^{2}{ }_{\text {com }}-\left(n_{1}+n_{2}-4\right) * S^{2}{ }_{\text {pool }}}{2} \\
F=\frac{S^{2}{ }_{\text {imp }}}{S^{2}{ }_{\text {pool }}}
\end{gathered}
$$


single regression line encompassing both groups. All subgroups comparing LL and pelvic tilt or hip flexion were compared in this manner regardless of a statistically significant correlation. A 95\% confidence level was used for all statistical tests.

\section{Results}

The hyper-tensioned clavicle strap was able to decrease LL (mean \pm SD) $-4 \pm 2$ deg throughout the whole gait cycle in 9 of the 10 subjects, one subject showed an increase in LL. Since no other individuals showed an increase in lordosis, no comparisons could be made for this condition. The only statistically significant change, when compared to Holm-Sidak corrected p-critical values, was the decrease in LL $(\mathrm{p}<0.01)$. However, changes also occurred in plumbline $(1.3 \pm 0.9 \mathrm{~cm})$ and trunk-pelvic angle $(-1 \pm 1 \mathrm{deg})$ when compared to $\mathrm{p}$-critical $=0.05$ (Table 4-2).

Two modes of compensation were observed and determined to be statistically significant $(\mathrm{p}<0.0001)$ from each other, as determined by testing the coincidence of the two regression lines (Figure 4-2A). One mode, Compensation 1, shows LL decreasing with no or little change in pelvic tilt (slope \pm std. error) $0.06 \pm 0.04$. The other, Compensation 2, showed an approximately 2:1 ratio of decrease in LL to increase in pelvic tilt. These two groups also differed in that, individuals in Compensation 2 group presented with clinically tight hamstrings during the physical therapy evaluation and experienced more than \pm 3 degs of change in TK during gait. The Compensation 1 group had normal hamstring lengths and experienced less than \pm 2 deg change in TK. A statistically significant correlation was found in Compensation 2 (Figure 4-2B), correlating hip flexion to trunk-pelvic angle $(\mathrm{p}<0.05)$. This correlation suggests for every $1 \mathrm{deg}$ increase in trunk-pelvic angle there is a $0.39 \pm 0.03$ deg increase in hip flexion. However, the hip flexion - trunk-pelvic regressions of the Compensation 1 and Compensation 2 groups were not found to be statistically different, and it is possible there is no relationship between trunk-pelvic angle and hip flexion (Table 4-3). Additional regression plots relating pelvic tilt and hip flexion changes to changes in spine measures can be found in Appendix B (Figures B-4 to B-6).

Table 4-2: $\quad$ Changes Decreased Lumbar Lordosis from Normal during Gait

\begin{tabular}{lccccc}
\hline \multicolumn{1}{c}{$\mathrm{N}=9$} & $\begin{array}{c}\text { Lumbar } \\
\text { Lordosis }\end{array}$ & $\begin{array}{c}\text { Pelvic } \\
\text { Tilt }\end{array}$ & Plumbline & $\begin{array}{c}\text { Hip } \\
\text { Flexion }\end{array}$ & $\begin{array}{c}\text { Trunk-Pelvic } \\
\text { Angle }\end{array}$ \\
\hline Mean $\pm \mathrm{SD}$ & $-4 \pm 2^{\circ}$ & $1 \pm 1^{\circ}$ & $1.3 \pm 0.9 \mathrm{~cm}$ & $1 \pm 1^{\circ}$ & $-1 \pm 1^{\circ}$ \\
Range & -8 to $-2^{\circ}$ & -1 to $3^{\circ}$ & -1 to $2 \mathrm{~cm}$ & -2 to $1^{\circ}$ & -1 to $3^{\circ}$ \\
P-Value & $\mathbf{0 . 0 0 8}^{*}$ & 0.674 & $0.015^{*}$ & 0.110 & $0.044^{*}$ \\
P-Critical & 0.010 & 0.050 & 0.013 & 0.025 & 0.017 \\
\hline
\end{tabular}

Notes:

Measures $=$ Orthotic - Normal

Bold indicates significant differences when compared to corrected P-Critical value

* denotes values considered significantly different when compared to $\mathrm{P}=0.05$ 

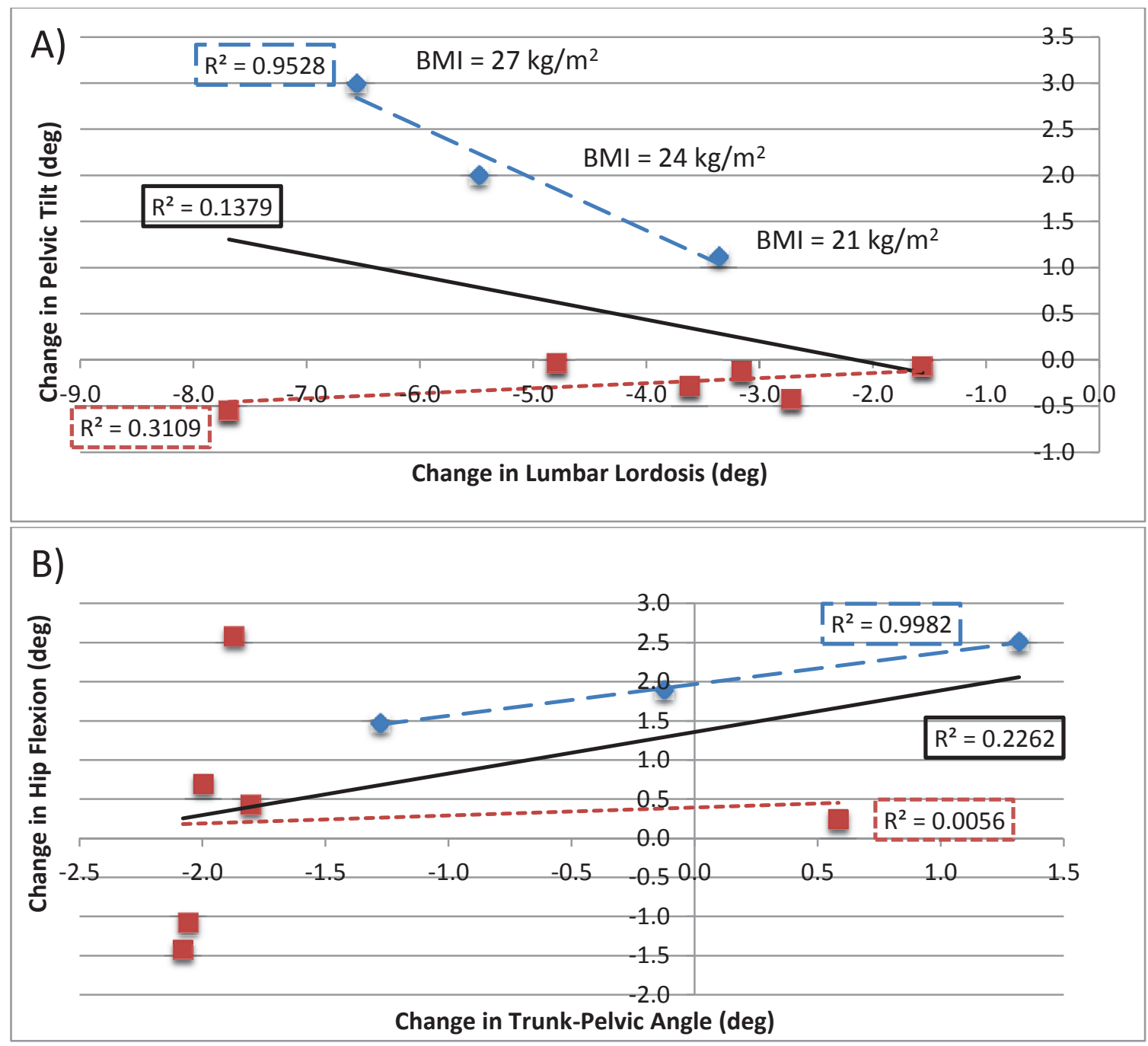

\section{Figure 4-2: $\quad$ Significant Correlations during Gait}

A) Change in Pelvic Tilt vs Change in Lumbar Lordosis Correlations during Gait B) Change in Hip Flexion vs Change in Trunk-Pelvic Angle Correlations during Gait Red squares represent the Compensation 1 group.

Blue diamonds represent the Compensation 2 group.

The lines represent linear regressions for their respective groups: blue large dashed line $=$ red short dashed $=$ Compensation 1 , Compensation 2, and solid black $=$ Overall . 
Table 4-3: $\quad$ Summary of Gait Correlations

\begin{tabular}{|c|c|c|c|c|c|}
\hline Correlations & $\mathrm{R}^{2}$ & Adjusted $\mathrm{R}^{2}$ & S.E.E & ${ }^{*}$ Slope \pm Std. Error & $\ddagger \mathrm{P}-$ Value \\
\hline PT vs LL Overall & 0.138 & 0.015 & 1.24 & $-0.2 \pm 0.2$ & 0.325 \\
\hline $\begin{array}{l}\text { PT vs LL } \\
\text { Compensation } 1\end{array}$ & $\underline{0.332}$ & $\underline{0.15}$ & $\underline{0.21}$ & $\underline{0.06 \pm 0.04}$ & $\underline{0.241}$ \\
\hline $\begin{array}{l}\text { PT vs LL } \\
\text { Compensation } 2\end{array}$ & $\underline{0.957}$ & $\underline{0.914}$ & $\underline{0.28}$ & $\underline{-0.5 \pm 0.1}$ & $\underline{0.133}$ \\
\hline PT vs PL Overall & 0.155 & 0.034 & 1.23 & $0.5 \pm 0.5$ & 0.295 \\
\hline $\begin{array}{l}\text { PT vs PL } \\
\text { Compensation } 1\end{array}$ & 0.094 & -0.133 & 0.24 & $0.08 \pm 0.1$ & 0.556 \\
\hline $\begin{array}{l}\text { PT vs PL } \\
\text { Compensation } 2\end{array}$ & 0.073 & -0.855 & 1.29 & $0.2 \pm 0.9$ & 0.826 \\
\hline PT vs T-P Overall & 0.367 & 0.276 & 1.06 & $0.6 \pm 0.3$ & 0.084 \\
\hline $\begin{array}{l}\text { PT vs T-P } \\
\text { Compensation } 1\end{array}$ & 0.008 & -0.240 & 0.25 & $-0.02 \pm 0.1$ & 0.869 \\
\hline $\begin{array}{l}\text { PT vs T-P } \\
\text { Compensation } 2\end{array}$ & 0.188 & -0.623 & 1.21 & $0.3 \pm 0.7$ & 0.714 \\
\hline HF vs LL Overall & 0.046 & -0.090 & 1.51 & $0.2 \pm 0.3$ & 0.579 \\
\hline $\begin{array}{l}\text { HF vs LL } \\
\text { Compensation } 1\end{array}$ & 0.437 & 0.297 & 1.20 & $0.5 \pm 0.3$ & 0.153 \\
\hline $\begin{array}{l}\text { HF vs LL } \\
\text { Compensation } 2\end{array}$ & 0.307 & -0.386 & 0.59 & $-0.2 \pm 0.3$ & 0.626 \\
\hline HF vs PL Overall & 0.005 & -0.137 & 1.55 & $0.1 \pm 0.6$ & 0.852 \\
\hline $\begin{array}{l}\text { HF vs PL } \\
\text { Compensation } 1\end{array}$ & 0.007 & -0.241 & 1.60 & $-0.1 \pm 0.8$ & 0.875 \\
\hline $\begin{array}{l}\text { HF vs PL } \\
\text { Compensation } 2\end{array}$ & 0.633 & 0.265 & 0.43 & $-0.4 \pm 0.3$ & 0.415 \\
\hline HF vs T-P Overall & 0.217 & 0.105 & 1.37 & $0.5 \pm 0.4$ & 0.206 \\
\hline $\begin{array}{l}\text { HF vs T-P } \\
\text { Compensation } 1\end{array}$ & 0.004 & -0.245 & 1.60 & $0.09 \pm 0.7$ & 0.905 \\
\hline $\begin{array}{l}\text { HF vs T-P } \\
\text { Compensation } 2\end{array}$ & 0.995 & 0.99 & 0.05 & $0.39 \pm 0.03$ & 0.045 \\
\hline
\end{tabular}

Notes:

Underlined groups were found to be statistically different on the $95 \%$ interval.

Compensation 1 and Compensation 2 was determined as statistically different $p<0.0001$. A Mann-Whitney U Test found pelvic tilt of Compensation 1 and Compensation 2 to be statistically different $\mathrm{p}=0.024$.

Compensation $1(\mathrm{~N}=6)$, Compensation $2(\mathrm{~N}=3)$, Overall $(\mathrm{N}=9)$

* Slopes and Standard Errors are for unstandardized coefficients

₹ P-Values were calculated using a Student's $-\mathrm{t}$ test of the regression slope. 


\section{Limitations and Assumptions}

It is important to interpret the results of this study with consideration to the study's limitations and assumptions. This study was limited to young, healthy, adults with no history of spinal or lower extremity pathologies or surgeries. Gait was performed in a normal manner and at a self-selected pace. The clavicle strap was not tightened to a specific tension, and it was assumed that both sides were tightened equally. Changes in spinal curvature due to the hyper-tensioned clavicle strap were subject dependent, i.e. subjects were not all altered to a specific lordosis angle. The resulting changes in posture were generally small, less than 10 degs. The opto-electronic cameras were on average calibrated to a resolution of $0.4 \mathrm{~mm}$, which corresponds to approximately $0.5 \mathrm{deg}$ resolution depending marker distance. Another limitation is that the location of the hip joint center had to be approximated using anthropomorphic equations. However, since changes in hip flexion were investigated, errors in position would be subtracted out and would not be a factor in this study.

\section{Discussion}

With the addition of the hyper-tensioned clavicle strap, 9 of the subjects experienced a decrease in LL (mean \pm SD) $-4 \pm 2$ deg. While this was a statistically significant change $(\mathrm{p}=0.008)$, there was little to no change in anterior pelvic tilt $1 \pm 1 \mathrm{deg}$. Overall, as LL decreased and the spine flattened, plumbline increased and the torso assumed a flexed position.

A different compensation trend appeared among 3 of the 9 subjects tested. These 3 exhibited an approximately 2:1 change in LL to change in pelvic tilt. This trend was found to be significantly different than the other 6 and is visually distinct in Figure 4-1A. These 3 individuals also presented with tight hamstrings, and changes in TK greater than \pm 3 deg. As the hamstrings connect the pelvis to the femur and tibia, shorter (tighter) hamstrings should decrease pelvic tilt [58]. The popliteal angle, a measure of hamstring tightness and length as determined by the physical therapist, for the 3 subjects ( 2 male, 1 female) were $-25 \mathrm{deg},-30 \mathrm{deg}$, and $-25 \mathrm{deg}$ respectively. These are clinically classified as tight, but would not inhibit a person's ability to walk. By allowing their pelvis to rotate forward the subjects are lengthening the hamstrings, and could be utilizing muscle elasticity to aid in compensation. Another interesting note is the fact that increased pelvic tilt for subjects in Compensation 2 corresponds with their BMI. The 3, 2, and 1 deg changes correspond to BMI values 27,24 , and $21 \mathrm{~kg} / \mathrm{m}^{2}$ respectively.

Even with a 6 deg decrease in LL and a 2 deg increase in pelvic tilt, one Compensation 2 subject was able to return to their original plumbline by decreasing their TK by $3 \mathrm{deg}$. The other two subjects were not able to restore their original plumbline, and their TK was increased by 5 and 6 deg. It is most likely that these two were unable to flatten their thoracic spine due to the hyper-tensioned clavicle strap, and as a result their plumbline was shifted more than $2 \mathrm{~cm}$ anteriorly. Shifting the center of gravity forward and shifting the hip backwards increases demands on the hip joint. Similarly, increasing 
the forward tilt of the torso increases demands on the back muscles. It is possible that these healthy young individuals were able to physically endure this offset, given it was only temporary and lasted approximately 30 minutes.

Another possible explanation for the increased pelvic tilt for the Compensation 2 group is the activation of erector spinae muscles. The erector spinae muscles are a group of muscles which act to extend the spine, and several of them anchor on the sacrum. It is possible that the two subjects with the greater than $2 \mathrm{~cm}$ increase in plumbline placed more demand on the erector spinae to hold that position. Similarly, it is possible that the third individual placed more demands on these muscles to turn to a normal plumbline. This was the only individual to restore their plumbline to within $0.5 \mathrm{~cm}$ of normal, while the majority clustered around a $1.5 \mathrm{~cm}$ deviation. To better understand these different compensation modes, electromyography should be incorporated into future studies.

While this current study contains a small population with less than 10 degs and 3 $\mathrm{cm}$ change in LL and plumbline, respectively, it suggests that individuals with tight hamstrings may be more susceptible to changes in LL and plumbline or that they may be utilizing a different mode of compensation. If this trend is shown to be a common theme in future studies it may help explain why some individuals develop postoperative stooping, and could further aid in the development of patient-specific surgical planning. 


\section{CHAPTER 5. SPINO-PELVIC KINEMATICS OF SIT-TO-STAND AND STAND-TO-SIT ACTIVITIES UNDER DIFFERENT SAGITTAL ALIGNMENTS}

\section{Chapter Overview}

Flexion of the spine plays a crucial role in the ability to rise from, and return to, the seated position. At peak hip flexion, individuals are at the greatest risk for hip dislocations. As a result, patients who have undergone total hip arthroscopy are instructed not to flex their hips past 90 degs. Those who have undergone spine fusion surgery may have an altered spine curvature and limited motion. No study prior to this has sought to understand how an altered spine posture is compensated during the activities of sit-tostand (Si-St) and stand-to-sit (St-Si). This study utilized ten young, healthy subjects with no history of spine or low extremity pathologies or surgeries. The subjects were evaluated in a paired fashion performing Si-St and St-Si activities, using a $46 \mathrm{~cm}$ bench and a ten camera motion analysis laboratory. A custom hyper-tensioned clavicle strap was used to alter spine curvature. Intrasubject changes in lumbar lordosis, pelvic tilt, plumbline, hip flexion, and flexion of the trunk relative to the pelvic (Trunk-Pelvic angle) were analyzed at peak hip flexion. A Wilcoxon Signed-Rank Test was used to determine significance of changes, and a Student's-t test was used to determine significance of the linear regression slopes. This study found that during Si-St, changes in pelvic tilt had a 1:1 relationship with changes in plumbline $(\mathrm{p}<0.005)$. However, changes in plumbline only related to hip flexion $(1: 1.4, \mathrm{p}<0.005)$ when lordosis was decreased during Si-St. Similarly, plumbline only related to pelvic tilt $(1: 1, p=0.005)$ during $\mathrm{St}-\mathrm{Si}$ when lordosis was decreased. Changes in plumbline showed a 1:0.3 ratio with hip flexion during St-Si $(p=0.04)$.

\section{Background}

Rising from and descending to a seated position is a dynamic task, which requires complex muscle coordination and strength. The World Health Organization has classified the difficulty of getting in and out of chairs as a disability [59]. Rising from the seated position, and its inverse, is a common activity of daily living performed approximately 60 times a day [60]. and is an important function for independent living [61]. As a result it has been studied for decades encompassing a variety of testing equipment, techniques, and hypotheses [62]. However, few studies have shown interest in the spine beyond gross trunk motion $[46,50,51,63-66]$. Fewer still have sought to understand the role or impact of the spine on these motions [46, 51, 63-66]. As a result there is a dearth of knowledge when it comes to lumbar kinetics and kinematics.

In the Si-St task, the torso is propelled forward to generate momentum and aid in ascension [67]. It has been suggested that the propulsion is generated at the hips, and the torso follows as a gross structure. However, several studies demonstrate the lumbar spine flattens or extends during this phase of the Si-St cycle [46, 51, 65]. The contribution of the lumbar is widely variable between subjects [65] and is still not well understood. Koe 
et al (2010) showed shorter seat heights decrease the range of motion available for propulsive hip flexion and is compensated by increases in lumbar flexion [65]. This study suggests that at seat heights less than that of the person's knees, lumbar flexion becomes a more important generator of momentum.

The St-Si motion closely mirrors the Si-St motion [51, 67]; the lower extremity joints flex and eccentric muscle contraction is used to allow the individual's center of gravity fall in a controlled fashion until seat contact. The influence of the spine during this activity is even less understood and, to the best of the author's knowledge, has only been investigated in a few studies [50, 51, 67, 68] where the study populations were limited to asymptomatic and low back pain individuals. Shum et al. (2005 \& 2007) showed that lumbar motion precedes hip motion during the initial phase, and both structures exhibited a decrease in motion, moments, and powers in subjects with low back pain $[50,51]$. The low back pain individuals also showed an increase in total activity time [51].

Subjects who have undergone a THA show lower peak hip flexion angles (mean $=$ $82 \mathrm{deg}$ ) [69]. THA patients are instructed to not flex the hip past $90 \mathrm{deg}$ as they will be more prone to dislocations [70]. Approximately $27 \%$ of dislocations occur performing SiSt and St-Si [70]. Subjects who have undergone both SFS and THA may have more difficulty performing Si-St and St-Si activities due to their inability to flex their spine and limited hip flexion. If SFS patient compensate and recruit energy from the hips, the increase demands on the hip components and required increased hip flexion may make them more prone to dislocations.

To the author's knowledge no study has incorporated subjects with FSI or lumbar spinal fusions into a Si-St or St-Si study. As a result no quantitative knowledge is known about their function during this activity. Persons with a fused lumbar, or FSI, may not have the range of motion necessary to perform Si-St movements without use of their arms. At knee level or greater heights they may place greater demands on their hips, and may be a contributor the development of hip pathology.

The purpose of this study was to determine how changes in spinal position correspond with changes in pelvic tilt during Si-St and St-St, utilizing a hyper-tensioned clavicle strap. Preliminary investigations in our lab suggested that a hyper-tensioned clavicle strap would be able to consistently alter LL during gait, without obscuring the spinous processes or purchasing on the pelvis (Appendix C). Similarly, a radiographic validation study was conducted to ensure, that the hyper-tensioned clavicle strap alters LL (Chapter 3). Both the preliminary testing and validation study suggested that the clavicle strap could alter LL.We hypothesized that a subject would compensate an altered lumbar lordosis by altering their pelvic tilt and torso alignment to maintain a normal plumbline. 


\section{Methods}

\section{Participants}

Participants were recruited by word of mouth and by placing fliers about the UTHSC campus. Inclusion criteria were limited to persons 18 to 35 years of age with no history of spine/shoulder/lower limb injuries/defects, and could perform daily activities in the manner of a healthy adult. Pregnant women were also excluded from the study. Ten subjects were incorporated in this study (4 male, 6 female). A table containing the demographics of the population is provided in Table 5-1.

\section{Procedures}

Each subject was brought to the Motion Analysis Lab as part of the UTHSC, Department of Physical Therapy. The instruments used in the Motion Analysis Lab consisted of ten opto-electronic cameras (Qualisys AB, Gothenburg, Sweden), and 3 force plates (AMTI, Watertown, MA, USA). The cameras were calibrated to an accuracy of $< \pm 0.6 \mathrm{~mm}$ (mean $= \pm 0.4 \mathrm{~mm}$ ) of the calibration wand length, and the force plates were zeroed prior to each test.

Before retro-reflective markers were applied, the subjects would change into minimalist clothing (e.g. men: athletic shorts, women: athletic shorts and nonracerbacked sports bra), and a licensed physical therapist with 25 years experience would perform a standard physical therapy evaluation which included the following: height and weight measurements, the length of both legs as measured from the ASISs to their medial malleoli using a standard tape measure, assessment of joint mobility, muscle strength and flexibility. This evaluation was to screen for any undiagnosed physical limitations.

The same physical therapist then applied thirty-two $12.7 \mathrm{~mm}$ reflective markers over anatomical landmarks of the torso and bilaterally over the lower extremities Figure 4-1A. All subjects had 9 markers placed on their torso: left and right acromion (ACR), and spinous processes C7, T4, T7, T10, T12, L2, L4; 4 markers defined the pelvis: right and left ASISs and PSISs; 3 markers over the sacrum (SACR): one over S2, and two inferiorly (SL, SR); a total of 16 markers were placed bilaterally over the medial and lateral epicondyles (MED \& LATFEMCON) and malleoli (MED \& LATMALL), calcaneus (CALC), dorsum (DRSM), $5^{\text {th }}$ metatarsal head $(5 \mathrm{TH})$, as well as the great toe

Table 5-1: $\quad$ Summary of Subject Demographics (Mean \pm SD)

\begin{tabular}{lcccc}
\hline \multicolumn{1}{c}{ Subjects } & Height $(\mathrm{cm})$ & Mass $(\mathrm{kg})$ & BMI $\left(\mathrm{kg} / \mathrm{m}^{2}\right)$ & Age $($ years $)$ \\
\hline Male $(\mathrm{N}=4)$ & $173 \pm 8$ & $76 \pm 7$ & $25.7 \pm 1.3$ & $28 \pm 6$ \\
Female $(\mathrm{N}=6)$ & $168 \pm 5$ & $63 \pm 3$ & $22.2 \pm 0.8$ & $24 \pm 3$ \\
Total $(\mathrm{N}=10)$ & $171 \pm 6$ & $70 \pm 8$ & $23.9 \pm 2.1$ & $26 \pm 4$ \\
\hline
\end{tabular}


(GTOE) were used to define the lower extremities. Clusters of 4 markers secured to a plastic base were attached laterally to the thighs and shanks using self-adhering elastic wraps to aid in tracking segment motion. Additional $9.5 \mathrm{~mm}$ markers were added in between marked processes when possible (F\#). These markers served to further define the curvature of the full spine as well as to mark lumbar and thoracic apexes. All markers were attached using double-sided tape, and clothing was taped such that the fabric would not obscure markers. Duct tape was added to spinal and pelvic markers, to ensure their fixation.

After the markers were attached the barefoot subject was positioned, in the middle of a specially designed 25 foot-long platform instrumented with three force plates, with each foot on a force plate. The subject was instructed to stand in a normal relaxed position with their hands held in front of their face, as if they were doing a pull-up, and elbows brought anteriorly away from their torso. This position was used because it allowed full visibility of markers and served as part of a separate study. The subject was recorded twice for one second in the standing position. These static captures are used to generate a 6 degree of freedom (DOF) linked segment model.

Medial markers were then removed, and the subject was instructed to walk back and forth along the platform. After several practice trials to allow the subject to adjust to the test conditions, a bench $46 \mathrm{~cm}$ high $(100 \pm 6 \%$ of subject's knee height) was then positioned in the middle of the capture volume such that each foot of the subject would contact a separate force plate. This seat height was chosen, because it is believed to be a height commonly encountered during daily living [61]. The subject was instructed to start in a seated position with feet positioned comfortably. To prevent the use of their arms, the subjects were instructed to keep their arms crossed comfortably across their chest during the trials. The subject was then instructed to perform several practice trials to accommodate them to the new task. This also allowed the investigators to determine if the subject's feet were positioned cleanly on each force plate. If a portion of the foot was over the edge of a force plate the bench would be repositioned until a clean foot placement was achieved. If the bench was moved the subject was given more practice trails. Once the subject was comfortable, and the position correct, the subject was instructed to rise from the bench, count to two, return to sitting, count to two, and to perform this task at a comfortable self-selected pace. After a trial was completed, the subject was then asked to walk about the platform and to return to the bench. This served to randomize foot position. The subject was recorded performing this task twice, each for 30 seconds. This resulted in approximately 4 ascents and descents per trial. A trial was considered successful if the subject was able to perform the task without using their hands and pelvic and spine markers were visible. If pelvic or spine markers were lost, garments were repositioned or taped to prevent obstruction.

With the normal session complete, the subject would be equipped with a modified clavicle strap. The strap was designed and manufactured with the aid of Spears Orthosis and Prosthetics to hold the shoulders back with minimal obstruction of the spinous processes (Figure 2-1). The straps were tightened to the limit of the subject's comfort level, and the position of each strap was marked using an indelible ink pen. If the clavicle 
strap obscured the $\mathrm{C} 7$ or T4 marker, it was rotated about the shoulders until the marker was no longer obscured. However, due to skin folds and sports bra straps some thoracic measurements were lost.

The subject was then instructed to walk along the platform to allow them to become familiar with the device, and the altered posture. Once the subject was comfortable with the new posture, they were again brought to the middle of the capture volume and two static measurements were collected. The subjects then repeated the bench activity in the same manner as previously described.

\section{Kinematic Analysis}

All activities were recorded at $100 \mathrm{~Hz}$ using 10 opto-electric cameras (Qualisys $\mathrm{AB}$, Gothenburg, Sweden), marker position was interpolated over a maximum of 10 frames using a $3^{\text {rd }}$ order polynomial, and low pass filled at $7 \mathrm{~Hz}$ using a $4^{\text {th }}$ order digital Butterworth filter. Marker position of static trials was processed in the same manner at $2 \mathrm{~Hz}$. GRFs and center of pressures were low pass filtered at $15 \mathrm{~Hz}$. Kinematics and GRFs were recorded using Qualysis 8.9 (Qualysis AB, Gothenburg, Sweden) and then exported to Visual3D version 5 (C-Motion Inc., Germantown, MD) for processing.

The sagittal plumbline, was measured by the anterior displacement of the $\mathrm{C} 7$ marker from the S2 sacrum marker. TK and LL were measured by the acute angle formed of the C7-T4/ T7-10 lines and the T12-L2/L4-S2 lines, respectively, as described by Fowler et al (2004). Pelvic tilt was calculated in the sagittal plane by the angle of the line segment connecting the midpoint of the ASIS markers to midpoint of the PSIS markers. as referenced from horizontal. The sagittal plane was defined as the vertical plane (with respect to gravity) which passes through the midpoints of the PSISs and ASISs (Figure 4-1C). The flexion of the hips was calculated by the rotation of the line segment connecting the hip joint centers to the midpoint of the ipsolateral epicondyle markers, as referenced by mediolaterial axis of the pelvis. Further description of pelvic and spine measures can be found in Chapter 1.

Since the Si-St and St-Si trials were recorded continuously over 30 seconds, it was necessary to define boundary conditions for each cycle Table 5-2. Unfortunately, some individuals had gaps in marker position too large to make comparing full cycles meaningful at this point in time.

The parameters of interest were investigated at peak right (arbitrarily chosen) hip flexion in the Si-St and St-Si cycles. The first sitting and standing hip flexion events for each trial were ignored for all subjects. These events were ignored to prevent new task aberrations from appearing in the data. LL, plumbline, pelvic tilt, TK, and right hip flexion were arithmetically averaged at peak right hip flexion for the normal condition, minimum of 5. The difference in LL, plumbline, pelvic tilt, TK, and hip flexion of each orthotic trial from the mean normal value was calculated. For both $\mathrm{Si}-\mathrm{St}$ and $\mathrm{St}-\mathrm{Si}$, the three orthotic peak right hip flexions with the greatest average decrease and increase in 
Table 5-2: $\quad$ Cycle Boundary Conditions

\begin{tabular}{cll}
\hline Activity & \multicolumn{1}{c}{ Sit-Stand } & \multicolumn{1}{c}{ Stand-Sit } \\
\hline Initiation & $\begin{array}{l}\text { Horizontal velocity of } \mathrm{C} 7>0 \\
\mathrm{~mm} / \mathrm{s}\end{array}$ & $\begin{array}{l}\text { Horizontal velocity of Plumbline }> \\
3 \mathrm{~cm} / \mathrm{s}\end{array}$ \\
\multirow{2}{*}{ Termination } & Maximum Knee Extension & Horizontal velocity of $\mathrm{C} 7<0 \mathrm{~mm} / \mathrm{s}$ \\
\hline
\end{tabular}

lumbar lordosis (total 12) were determined. These values were exported to Microsoft Excel 2010 (Microsoft, Redmond, WA, USA) where the aforementioned parameters were arithmetically averaged over the three trials. Again, if at peak right hip flexion the orthotic trial did not contain all parameters of interest, excluding TK, the successive trial with the same direction of change was used.

\section{Statistical Analysis}

Prior to statistical analysis, UTHSC biostatistician Jim Wan was consulted to determine the most appropriate methods for our variables in question.

Even though 10 subjects participated in this study, not all subjects were evaluated in a given condition. For example, if a subject demonstrated only a decrease in lordosis during an activity they were not included in the increased lordosis group.

A Wilcoxon signed-rank tests with a Holms-Sidak correction was used to determine if the parameters of the orthotic condition were statistically different from those of the normal condition. Linear regression analysis was then performed comparing changings in LL, plumbline, and trunk-pelvic angle to changes in pelvic tilt and hip flexion. The slopes of each linear regression were then compared to zero using a Student's-t test. IBM SPSS Statistics 21 program (IBM Corporation, Armonk, NY) was used to perform Wilcoxon signed-rank tests, linear regression analysis, and Student's-t tests. Sub-groups were compared using Equation 4-1 and 2 when at least one group was found to have a statistically significant correlation.

\section{Results}

St-Si and Si-St activities were evaluated at peak hip flexion, as determined by tracking the motion of the pelvis and thigh. Peak hip flexion during St-Si and Si-St activities in the unaltered condition occurred at (mean \pm SD) $92 \pm 9$ deg and $90 \pm 9$ deg, respectively. Average angle values during St-Si and Si-St activities differed by at most 3 deg (Table 5-3). On average, the subjects were consistent during these activities. The average variability of a subject during these activities was \pm 1 deg for angle measures and $\pm 0.1 \mathrm{~cm}$ for plumbline (Table 5-3). The variability in these measures increased substantially after donning of the hyper-tensioned clavicle trap, increasing up to 2 deg for $\mathrm{LL}$ and pelvic tilt, and $2 \mathrm{~cm}$ in plumbline. 
Table 5-3: Normal Values at Peak Hip Flexion during Stand-to-Sit and Sit-toStand Activities

\begin{tabular}{lccccc}
\hline \multicolumn{1}{c}{$\mathrm{N}=10$} & $\begin{array}{c}\text { Lumbar } \\
\text { Lordosis }\end{array}$ & $\begin{array}{c}\text { Pelvic } \\
\text { Tilt }\end{array}$ & Plumbline & Hip Flexion & $\begin{array}{c}\text { Trunk-Pelvic } \\
\text { Angle }\end{array}$ \\
\hline $\begin{array}{l}\text { St-Si } \\
\text { Mean } \pm \mathrm{SD}\end{array}$ & $17 \pm 9^{\circ}$ & $26 \pm 9^{\circ}$ & $2.9 \pm 0.5 \mathrm{~cm}$ & $92 \pm 9^{\circ}$ & $79 \pm 9^{\circ}$ \\
$\mathrm{St}-\mathrm{Si}$ & $1^{\circ}$ & $1^{\circ}$ & $0.1 \mathrm{~cm}$ & $1^{\circ}$ & $1^{\circ}$ \\
$\begin{array}{l}\text { Average SD } \\
\text { Si-St }\end{array}$ & $14 \pm 11^{\circ}$ & $23 \pm 8^{\circ}$ & $2.8 \pm 0.5 \mathrm{~cm}$ & $90 \pm 9^{\circ}$ & $78 \pm 10^{\circ}$ \\
$\begin{array}{l}\text { Mean } \pm \mathrm{SD} \\
\text { Si-St }\end{array}$ & $1^{\circ}$ & $1^{\circ}$ & $0.1 \mathrm{~cm}$ & $1^{\circ}$ & $1^{\circ}$ \\
Average SD & & & & & \\
\hline
\end{tabular}

\section{Stand-to-Sit}

The change in LL at peak hip flexion during St-Si was statistically significant in both increased and decreased lordosis conditions (mean $\pm \mathrm{SD}$ ) $3 \pm 3 \mathrm{deg}$ and $-3 \pm 1 \mathrm{deg}$, respectively $(\mathrm{p}<0.01$, Table 5-4). While not statistically significant when compared to a Holm-Sidak correction: changes in plumbline, hip flexion, and trunk-pelvic angle, exhibited low $p$-values $(\mathrm{p}<0.05)$ in the decreased lordosis condition (Decreased). In this condition the plumbline, hip flexion, and trunk-pelvic angle changed $2.1 \pm 2.1 \mathrm{~cm}, 1 \pm 2$ $\mathrm{deg}$, and $-2 \pm 2 \mathrm{deg}$, respectively. In the increased lordosis condition (Increased) the trunk-pelvic angle increased $3 \pm 3 \mathrm{deg}$ and also corresponded with a low, non-statistically significant, p-value.

Several significant correlations were found at peak hip flexion during St-Si (Table 5-5). The increase in lumbar lordosis regression was statistically different than the decrease lordosis regression when relating pelvic tilt to plumbline $(p<0.01$, Figure 5-1A). The correlation between pelvic tilt and plumbline was statistically significant $(\mathrm{p}=0.048)$ for the regression spanning both conditions, termed "Overall", as well for the Decreased condition $(\mathrm{p}=0.005)$. For the Overall regression a $3 \mathrm{~cm}$ change in plumbline corresponded to a $1 \mathrm{deg}$ change in pelvic tilt; however in the Decreased condition the plumbline to pelvic tilt ratio approached 1:1 (Table 5-5). A similar trend was present when correlating plumbline to hip flexion (Figure 5-1B); the Overall and Decreased correlations were statistically significant, $\mathrm{p}=0.045$ and 0.039 respectively, and exhibited an approximately 10:3 and 10:6 plumbline to hip flexion ratio, respectively. However, in the case of the plumbline: hip flexion regressions, the Increased group and Decreased group were not statistically different groups. 
Table 5-4: Changes from Normal in Sit-to-Stand and Stand-to-Sit Activities at Peak Hip Flexion

\begin{tabular}{|c|c|c|c|c|c|}
\hline $\mathrm{N}=9$ & $\begin{array}{l}\text { Lumbar } \\
\text { Lordosis }\end{array}$ & $\begin{array}{l}\text { Pelvic } \\
\text { Tilt }\end{array}$ & Plumbline & $\begin{array}{l}\text { Hip } \\
\text { Flexion }\end{array}$ & $\begin{array}{c}\text { Trunk-Pelvic } \\
\text { Angle }\end{array}$ \\
\hline \multicolumn{6}{|c|}{ Max Lordosis Decrease from Normal Stand-to-Sit } \\
\hline Mean $\pm \mathrm{SD}$ & $-3 \pm 1^{\circ}$ & $1 \pm 3^{\circ}$ & $2.1 \pm 2.1 \mathrm{~cm}$ & $1 \pm 2^{\circ}$ & $-2 \pm 2^{\circ}$ \\
\hline Range & -4 to $0^{\circ}$ & -4 to $4^{\circ}$ & -1 to $5 \mathrm{~cm}$ & -1 to $3^{\circ}$ & -5 to $1^{\circ}$ \\
\hline P-Value & $0.008 *$ & 0.441 & $0.021 *$ & $0.044 *$ & $0.021 *$ \\
\hline P-Critical & 0.010 & 0.050 & 0.017 & 0.025 & 0.013 \\
\hline \multicolumn{6}{|c|}{ Max Lordosis Increase from Normal Stand-to-Sit } \\
\hline Mean $\pm \mathrm{SD}$ & $3 \pm 3^{\circ}$ & $1 \pm 2^{\circ}$ & $-1.6 \pm 2.7 \mathrm{~cm}$ & $1 \pm 2^{\circ}$ & $3 \pm 3^{\circ}$ \\
\hline Range & 0 to $9^{\circ}$ & -2 to $3^{\circ}$ & -6 to $2 \mathrm{~cm}$ & -2 to $3^{\circ}$ & -3 to $8^{\circ}$ \\
\hline P-Value & $0.008 *$ & 0.213 & 0.173 & 0.208 & $0.028 *$ \\
\hline P-Critical & 0.010 & 0.050 & 0.017 & 0.025 & 0.013 \\
\hline \multicolumn{6}{|c|}{ Max Lordosis Decrease from Normal Sit-to-Stand } \\
\hline Mean $\pm \mathrm{SD}$ & $-3 \pm 1^{\circ}$ & $2 \pm 3^{\circ}$ & $2.4 \pm 2.3 \mathrm{~cm}$ & $1 \pm 4^{\circ}$ & $-1 \pm 2^{\circ}$ \\
\hline Range & -5 to $-1^{\circ}$ & -2 to $10^{\circ}$ & 0 to $8 \mathrm{~cm}$ & -2 to $10^{\circ}$ & -5 to $2^{\circ}$ \\
\hline P-Value & $0.008 *$ & 0.11 & 0.011* & 0.374 & 0.086 \\
\hline P-Critical & 0.010 & 0.025 & 0.013 & 0.050 & 0.017 \\
\hline \multicolumn{6}{|c|}{ Max Lordosis Increase from Normal Sit-to-Stand } \\
\hline Mean $\pm \mathrm{SD}$ & $2 \pm 1^{\circ}$ & $3 \pm 3^{\circ}$ & $-0.5 \pm 2.3 \mathrm{~cm}$ & $3 \pm 3^{\circ}$ & $3 \pm 2^{\circ}$ \\
\hline Range & 0 to $4^{\circ}$ & -1 to $7^{\circ}$ & -3 to $3 \mathrm{~cm}$ & -2 to $6^{\circ}$ & 1 to $5^{\circ}$ \\
\hline P-Value & $0.008 *$ & $0.011 *$ & 0.514 & $0.021 *$ & $0.008 *$ \\
\hline P-Critical & 0.010 & 0.017 & 0.050 & 0.025 & 0.013 \\
\hline
\end{tabular}

Notes:

Measures $=$ Orthotic - Normal

Bold indicates significant differences when compared to corrected P-Critical value * denotes values considered significantly different when compared to $\mathrm{P}=0.05$ Bench Height $=46 \mathrm{~cm}(88-107 \%$ of knee height $)$ 
Table 5-5: $\quad$ Summary of Correlations during Stand-to-Sit Activities

\begin{tabular}{lccccc}
\hline \multicolumn{1}{c}{ Stand-to-Sit } & $\mathrm{R}^{2}$ & Adjusted R $^{2}$ & S.E.E & *Slope \pm Std. Error & $\ddagger$ P-Value \\
\hline PT vs LL Overall & 0.004 & -0.058 & 2.2 & $0.0 \pm 0.2$ & 0.803 \\
PT vs LL Increase & 0.001 & -0.141 & 1.7 & $0.0 \pm 0.2$ & 0.928 \\
PT vs LL Decrease & 0.022 & -0.118 & 2.8 & $0.3 \pm 0.7$ & 0.702 \\
PT vs PL Overall & 0.222 & 0.174 & 1.9 & $0.3 \pm 0.2$ & $\mathbf{0 . 0 4 8}$ \\
PT vs PL Increase & $\underline{0.313}$ & $\underline{0.215}$ & $\underline{1.4}$ & $\underline{0.3 \pm 0.2}$ & $\underline{0.117}$ \\
PT vs PL Decrease & $\underline{0.699}$ & $\underline{0.656}$ & $\underline{1.6}$ & $\underline{1.0 \pm 0.3}$ & $\underline{\mathbf{0 . 0 0 5}}$ \\
PT vs T-P Overall & 0.000 & -0.062 & 2.2 & $0.0 \pm 0.1$ & 0.976 \\
PT vs T-P Increase & 0.007 & -0.135 & 1.7 & $0.0 \pm 0.2$ & 0.831 \\
PT vs T-P Decrease & 0.005 & -0.138 & 2.8 & $0.1 \pm 0.5$ & 0.863 \\
HF vs LL Overall & 0.057 & -0.002 & 1.9 & $-0.1 \pm 0.1$ & 0.341 \\
HF vs LL Increase & 0.185 & 0.069 & 2.0 & $-0.3 \pm 0.3$ & 0.248 \\
HF vs LL Decrease & 0.190 & 0.074 & 1.7 & $0.6 \pm 0.5$ & 0.241 \\
HF vs PL Overall & 0.228 & 0.180 & 1.7 & $0.3 \pm 0.1$ & $\mathbf{0 . 0 4 5}$ \\
HF vs PL Increase & 0.155 & 0.034 & 2.0 & $0.3 \pm 0.3$ & 0.295 \\
HF vs PL Decrease & 0.477 & 0.403 & 1.4 & $0.6 \pm 0.2$ & $\mathbf{0 . 0 3 9}$ \\
HF vs T-P Overall & 0.044 & -0.016 & 1.9 & $-0.1 \pm 0.1$ & 0.403 \\
HF vs T-P Increase & 0.063 & -0.07 & 2.1 & $-0.2 \pm 0.3$ & 0.514 \\
HF vs T-P Decrease & 0.000 & -0.143 & 1.9 & $0.0 \pm 0.3$ & 0.964 \\
\hline
\end{tabular}

Notes:

$\mathrm{PT}=$ Pelvic Tilt, $\mathrm{LL}=$ Lumbar Lordosis, $\mathrm{PL}=$ Plumbline, $\mathrm{HF}=$ Hip Flexion, $\mathrm{T}-\mathrm{P}=$ TrunkPelvic Angle

* Slopes and Standard Errors are for unstandardized coefficients

₹ P-Values were calculated using a Student's-t test of the regression slope.

Bold p-values are less than 0.05

Underlined groups were found to be statistically different on the $95 \%$ interval using F-

Tests 

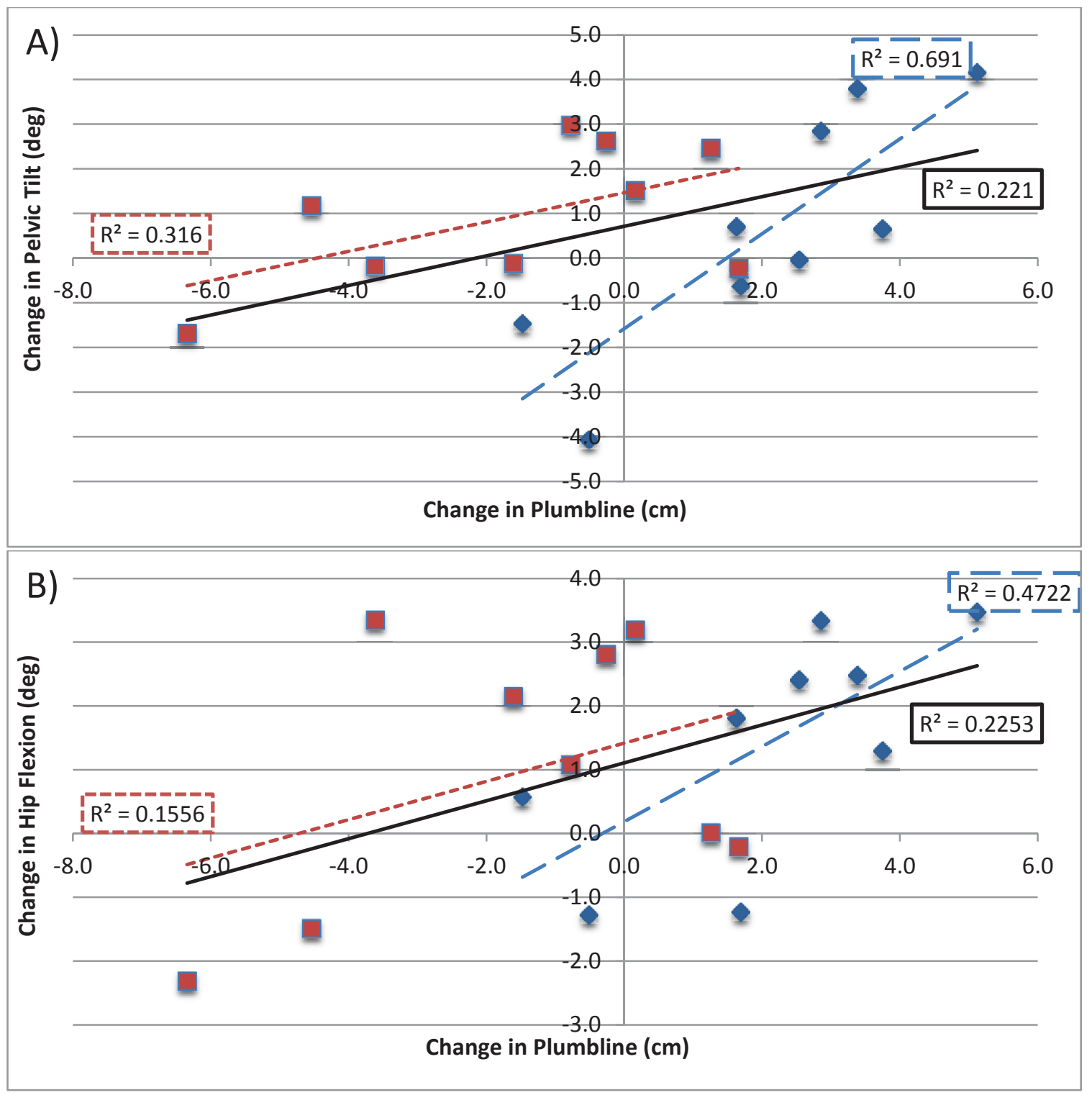

Figure 5-1: $\quad$ Significant Correlations during Stand-to-Sit at Peak Hip Flexion

A) Change in pelvic tilt relates to plumbline B) Change in Hip Flexion relates to plumbline

Blue diamonds represent the Decreased lumbar lordosis group.

Red squares represent the Increased in lumbar lordosis group.

The lines represent linear regressions for their respective groups: blue large dashed line $=$ Decreased, red short dashed $=$ Increased, and solid black $=$ Overall . 


\section{Sit-to-Stand}

The change in LL at peak hip flexion during Si-St was statistically significant in both increased $(2 \pm 1 \mathrm{deg})$ and decreased lordosis $(-3 \pm 1 \mathrm{deg})$ conditions (Table 5-6). For the decreased lordosis condition plumbline also significantly changed $2.4 \pm 2.3 \mathrm{~cm}$. In the increased lordosis condition plumbline was the only parameter which did not significantly change (Table 5-6).

This is interesting because pelvic tilt was found to be significantly correlated to plumbline for the Increased, Decreased, as well as Overall lordosis conditions during SiSt (Figure 5-2A). The Increased and Decreased regression groups were found to be statistically different form each other $(\mathrm{p}=0.001)$. The Increased group had an approximate 1:1 correlation (slope \pm Std. Error) $1.0 \pm 0.2(\mathrm{deg} / \mathrm{cm})$, while the Decreased group showed an approximate 1:1.25 correlation $1.2 \pm 0.3(\mathrm{deg} / \mathrm{cm})$. Similarly, the regression groups were found to be different in their hip flexion - plumbline correlations $(p=0.009$, Figure 5-2B). However, only the decreased lordosis group showed a significant correlation $1.4 \pm 0.3(\mathrm{deg} / \mathrm{cm})$.

Additional regression plots relating changes in gait measures be found in Appendix B (Figures B-7 to B-10).

\section{Limitations and Assumptions}

It is important to interpret the results of this study with consideration to the study's limitations and assumptions. This study was limited to young (18-35years), healthy (BMI $21.1-26.9 \mathrm{~kg} / \mathrm{m}^{2}$ ), adults with no history of spinal or hip pathologies or surgeries. Activities were performed in a normal manner and at a self-selected pace; therefore speed, foot position, and foot preferences were not controlled. Additionally, subjects' head position was not controlled, and subjects were free to look at the ground or wall during the activity. The clavicle strap was not tightened to specific tension, and it was assumed that both sides were tightened equally. Changes in spinal curvature due to clavicle strap was subject dependent, i.e. subjects were not all alter to a specific lordosis angle. The resulting changes in posture were generally small, less than 10 degs. The optoelectronic cameras were on average calibrated to a resolution of $0.4 \mathrm{~mm}$, which corresponds to approximately $0.5 \mathrm{deg}$ resolution, depending upon the distance between markers. Another limitation is that the location of the hip joint center had to be approximated using anthropomorphic equations. However, since changes in hip flexion were investigated, errors in position would be subtracted out and would not be a factor in this study. 
Table 5-6: $\quad$ Summary of Correlations during Sit-to-Stand Activities

\begin{tabular}{lccccc}
\hline \multicolumn{1}{c}{ Si-to-St (y vs x) } & $\mathrm{R}^{2}$ & Adjusted R $^{2}$ & S.E.E & *Slope \pm Std. Error & $\ddagger$ P-Value \\
\hline PT vs LL Overall & 0.004 & -0.058 & 3.0 & $0.1 \pm 0.2$ & 0.804 \\
PT vs LL Increase & 0.057 & -0.077 & 2.6 & $-0.4 \pm 0.6$ & 0.535 \\
PT vs LL Decrease & 0.131 & 0.007 & 3.3 & $-1.0 \pm 0.9$ & 0.339 \\
PT vs PL Overall & 0.337 & 0.295 & 2.5 & $0.6 \pm 0.2$ & $\mathbf{0 . 0 1 2}$ \\
PT vs PL Increase & $\underline{0.783}$ & $\underline{0.751}$ & $\underline{1.3}$ & $\underline{1.0 \pm 0.2}$ & $\underline{\mathbf{0 . 0 0 2}}$ \\
$\underline{\text { PT vs PL Decrease }}$ & $\underline{0.712}$ & $\underline{0.712}$ & $\underline{1.9}$ & $\underline{1.2 \pm 0.3}$ & $\underline{\mathbf{0 . 0 0 4}}$ \\
PT vs T-P Overall & 0.000 & -0.062 & 3.0 & $0.0 \pm 0.3$ & 0.988 \\
PT vs T-P Increase & 0.477 & 0.402 & 2.0 & $-1.0 \pm 0.4$ & $\mathbf{0 . 0 4}$ \\
PT vs T-P Decrease & 0.001 & -0.142 & 3.6 & $0.0 \pm 0.6$ & 0.950 \\
HF vs LL Overall & 0.018 & -0.043 & 3.3 & $0.1 \pm 0.3$ & 0.591 \\
HF vs LL Increase & 0.001 & -0.141 & 2.9 & $0.1 \pm 0.7$ & 0.926 \\
HF vs LL Decrease & 0.148 & 0.026 & 3.6 & $-1 \pm 1$ & 0.307 \\
HF vs PL Overall & 0.182 & 0.131 & 3.0 & $0.5 \pm 0.3$ & 0.078 \\
HF vs PL Increase & $\underline{0.224}$ & $\underline{0.113}$ & $\underline{2.6}$ & $\underline{0.6 \pm 0.4}$ & $\underline{0.196}$ \\
$\underline{\text { HF vs PL Decrease }}$ & $\underline{0.745}$ & $\underline{0.709}$ & $\underline{2.0}$ & $\underline{1.4 \pm 0.3}$ & $\underline{\mathbf{0 . 0 0 3}}$ \\
HF vs T-P Overall & 0.017 & -0.045 & 3.3 & $0.1 \pm 0.3$ & 0.611 \\
HF vs T-P Increase & 0.028 & -0.111 & 2.9 & $-0.3 \pm 0.6$ & 0.669 \\
HF vs T-P Decrease & 0.001 & -0.141 & 3.9 & $-0.1 \pm 0.7$ & 0.929 \\
\hline
\end{tabular}

Notes:

$\mathrm{PT}=$ Pelvic Tilt, $\mathrm{LL}=$ Lumbar Lordosis, $\mathrm{PL}=$ Plumbline, $\mathrm{HF}=$ Hip Flexion, $\mathrm{T}-\mathrm{P}=$ TrunkPelvic Angle

* Slopes and Standard Errors are for unstandardized coefficients

₹ P-Values were calculated using a Student's-t test of the regression slope.

Bold p-values are less than 0.05

Underlined groups were found to be statistically different on the $95 \%$ interval using F-

Tests 

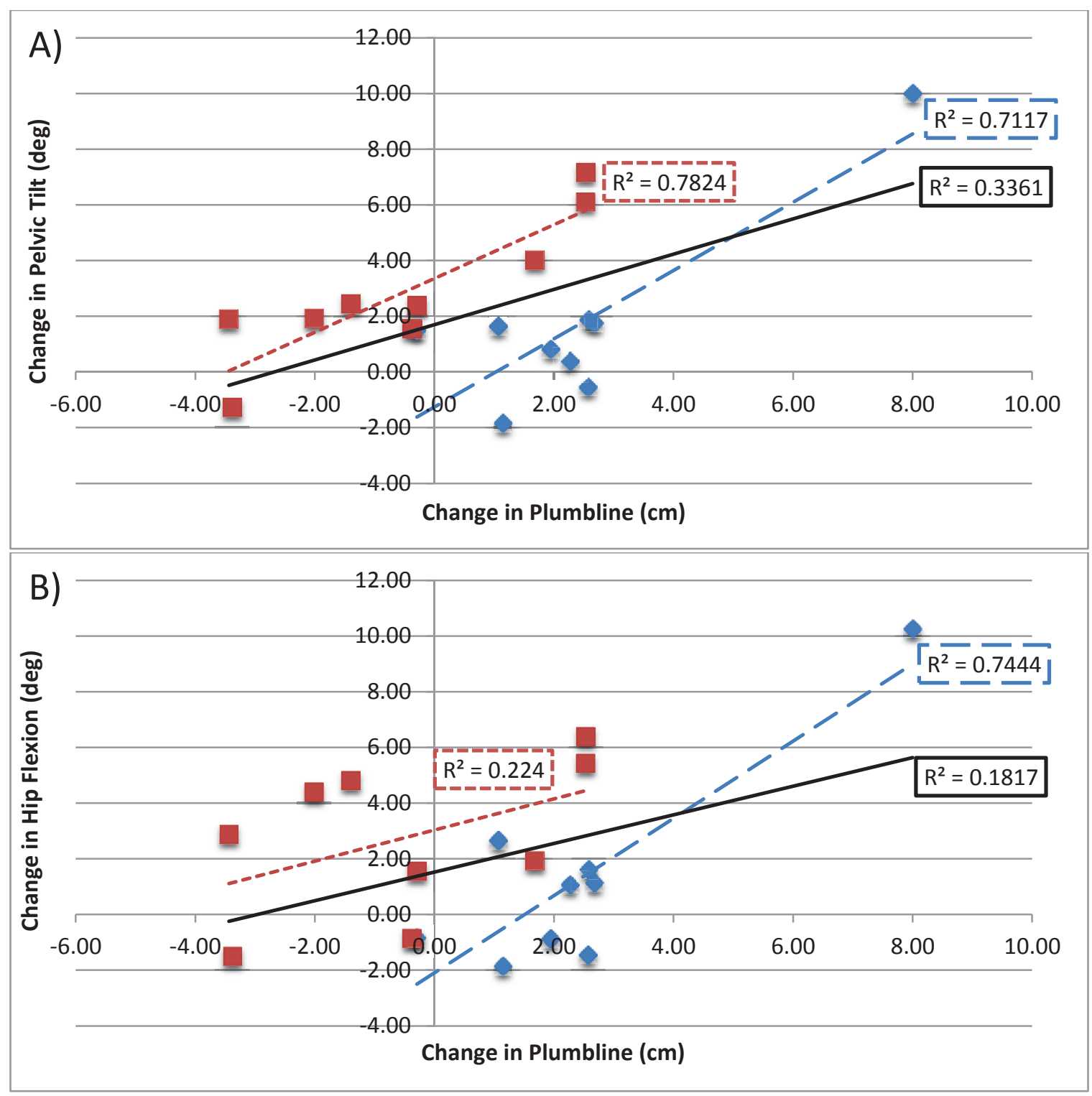

Figure 5-2: $\quad$ Significant Correlations during Sit-to-Stand at Peak Hip Flexion A) Change in pelvic tilt related to changes in plumbline B) Change in hip flexion related to change in plumbline

Blue diamonds represent the Decreased lumbar lordosis group. Red squares represent the Increased in lumbar lordosis group. The lines represent linear regressions for their respective groups: blue large dashed line $=$ Decreased, red short dashed $=$ Increased, and solid black $=$ Overall. 


\section{Discussion}

The purpose of this study was to determine how changes in spinal position correspond with changes in pelvic tilt during sit-to-stand and stand-to-sit, utilizing a hyper-tensioned clavicle strap. We hypothesized that a subject would compensate an altered lumbar lordosis by altering their pelvic tilt and torso alignment to maintain a normal plumbline.

We found that during the St-Si activity the hyper-tensioned clavicle strap was able to change lordosis from 9 to $-4 \mathrm{deg}$. While changes in lordosis were statistically significant $(p<0.008)$, there was no correlation to changes in pelvic tilt. The lack of relationship between the pelvis and lumbar was a surprising result given the strong evidence the relationship exists during static standing [2, 5, 6, 8, 14, 16, 18, 19]. The lack of relationship is most likely due to the instance investigated. At peak hip flexion during St-Si, the trunk, pelvis, and lower extremities are flexed and the muscles around the hips and knees are eccentrically contracting to lower the center of mass in a smooth controlled manner. At the instance of peak hip flexion the center of gravity is descending vertically and there is little motion required of the spine at this instance. It would make sense then that the pelvic tilt was found more related to the displacement of the center of gravity than to lordosis. When lordosis was decreased there was a significant 1:1 correlation between pelvic tilt and plumbline in centimeters $(p=0.005)$. This relationship suggested that the trunk and pelvis moved as a unit at peak hip flexion, and by increasing the plumbline the pelvis would equally increase. Interestingly, this 1:1 relationship did not translated to hip flexion. The decreased lumbar lordosis group showed a significant 1.0 $\mathrm{cm}$ : 0.6 deg plumbline to hip flexion ratio; so while pelvic tilt may increase $1 \mathrm{deg}$, hip flexion increases only $0.6 \mathrm{deg}$. This suggests that as the trunk and pelvis are rotated forward, the femur is more extended and additionally shifts the center of gravity farther forward. By shifting the center of mass farther forward, towards the knee joints, the eccentric demands of the knee are decreased and the subject is better able to descend in a slow or controlled manner. As the clavicle strap limits and alters the motion of the upper spine, it is possible that the balance mechanisms are altered as well. The Decreased and Increased groups were not found to be statistically different in their hip flexion responses due to an altered plumbline. This was primarily due to the large variance in the Increased group (Figure 5-1B). As a result, the overall trend suggests that a $1 \mathrm{~cm}$ increase in plumbline may result in a $0.3 \pm 0.1 \mathrm{deg}$ increase in hip flexion.

The hyper-tensioned clavicle strap was able to change lumbar lordosis 4 to -5 deg at peak hip flexion during Si-St $(\mathrm{p}=0.008)$. Interestingly, while the Decreased group also had a significant increase in plumbline, plumbline was the only measured parameter which did not significantly change in the Increased group (Table 5-4). Despite the lack of significant change in plumbline, there was a significant correlation between plumbline and pelvic tilt in the statistically distinct Increased and Decreased groups. The Increased group showed a nearly 1:1 correlation $(1.0 \pm 0.2)$ in plumbline to pelvic tilt, while the Decreased group showed a nearly 1:1.25 correlation $(1.2 \pm 0.3)$. The Decreased and Increased groups were significantly different when correlating hip flexion to plumbline as well. The Decreased group showed a significant 1.5:1 hip flexion to plumbline 
correlation $(1.4 \pm 0.31)$. In the Increased group of the Si-St activity, changes in pelvic tilt appear to be the predominate alteration. The changes in hip flexion and trunk-pelvic angle are nearly identical to pelvic tilt (Table 5-4), suggesting that changes in femur and torso flexion are minimal by comparison. This deduction is supported by the lack of significance and neutrality of the plumbline measure. Despite a statistically significant change in plumbline, plumbline has a nearly 1:1 correlation with pelvic tilt. Again, this correlation suggests that the torso and pelvis rotate about the hip joint as a unit at peak hip flexion. However, the fact that this correlation did not appear when relating plumbline to hip flexion suggests that the femur extension changes are subject-specific.

It is important to note that the correlation in the Decreased group in the $\mathrm{Si}-\mathrm{St}$ activity was strongly dependent upon a point which may be considered an outlier. The plumbline, pelvic tilt, and pelvic tilt/plumbline values were characterized as outliers $(>1.5 *$ interquartile range) when a boxplot was applied to the Decreased group. The only physical characteristics which set this individual apart were their mass and height. This subject was the tallest $(183 \mathrm{~cm})$ and heaviest $(85.7 \mathrm{~kg})$. The subject's height, while not an outlier, considerably skews the box plot of the group, and the subject's mass was found to be an outlier. Similarly, their BMI $\left(25.6 \mathrm{~kg} / \mathrm{m}^{2}\right)$ was not considered an outlier with the study group's $\left(23.9 \mathrm{~kg} / \mathrm{m}^{2}\right)$. This point was not removed from analysis because there were no physical or experimental characteristics which labeled the subject as abnormal, and given the small sample size and diversity of the subjects it is likely that this individual is still part of the normal population. The best explanation for this data point is due to the subject's increased height. Since the subject is taller, the seat height relative to their knee height was lower than the other subjects (88\% vs 94-107\%). It has been previously been reported that a decrease in seat height leads to an increase in torso, hip, knee, and ankle motion $[62,71]$, and that is what we are seeing. It is also interesting that this subject does not appear to be an outlier in any other activities, including the Increased condition during Si-St. With a larger spread of normal morphologies the plumbline to pelvic tilt correlation can be better understood and more confidently determined.

Due to the uncertainty in the plumbline correlations for the Decreased group, this thesis will leave further interpretation up to the reader, and will focus on the plumbline correlations for the Increased group during $\mathrm{Si}-\mathrm{St}$.

The fact that lumbar lordosis and pelvic tilt increased during Si-St while plumbline remained neutral brings to question the cause for the increases in lumbar lordosis and pelvic tilt. Levine et al (1996) showed that increases in pelvic tilt equally increase lumbar lordosis [28]. While this relationship is seen in our study when looking at the study population as a whole (Table 5-4), it is not apparent when examining the change in pelvic tilt verse change in lumbar lordosis plot (Figure 5-3). No regressions or data point suggests that a 1:1 change occurred.

If the correlations found in this study are shown to hold true, they have important implications for individuals with flatback deformity. For individuals with flatback deformity it is common to have a plumbline greater than $15 \mathrm{~cm}$, and as much as $30 \mathrm{~cm}$ [8]. Consider the individuals that have an increased plumbline of at least $12 \mathrm{~cm}$, and if 


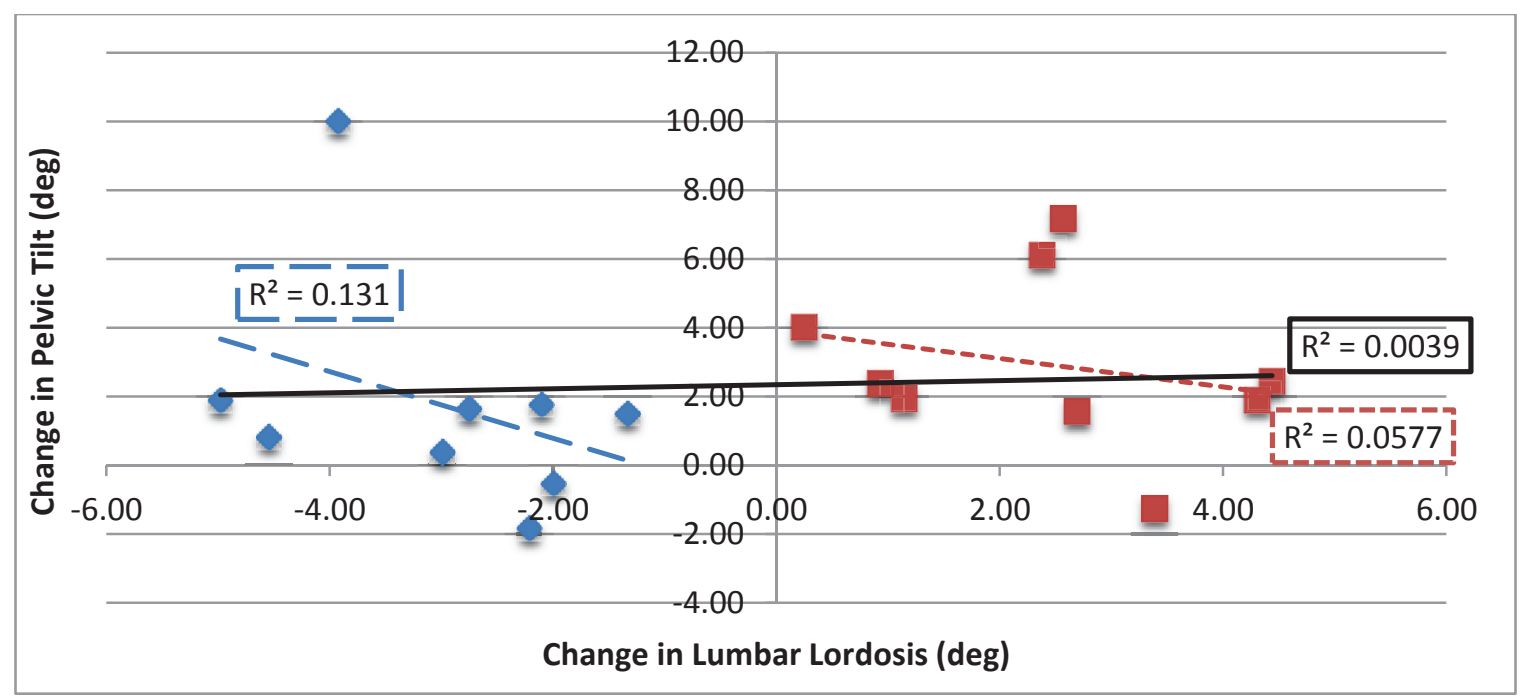

Figure 5-3: Changes in Pelvic Tilt and Lumbar Lordosis Regressions during Sitto-Stand

this translates directly to their St-Si activity, their pelvic tilt can increase equally as much. As previously mentioned, the safe zone for acetabular anteversion in regards to THA is 5$25 \mathrm{deg}[17,20]$, and acetabular anteversion has an approximately $-1: 1$ relationship with pelvic tilt [23]. So even if the surgeon places the acetabulum in the middle of the safezone (15 deg) most flatback individuals would be functioning outside of the safe-zone at peak hip flexion during Si-St. Since the forces and moments at peak hip flexion are near their max during this activity [72], this is when the function is most important and most demanding. 


\section{CHAPTER 6. GENERAL DISCUSSION}

\section{Introduction}

The goal of this project was to quantify the relationship between the spine and pelvis in the sagittal plane during static stance, ambulation, Si-St, and St-to-Si. We hypothesized that subjects would compensate changes in the lumbar spine by altering their pelvic tilt to restore a normal plumbline. This hypothesis was then tested using ten subjects between the ages of 18 and 35 with no history of spine or lower extremity pathologies or surgeries, and able to perform activities in the manner of a healthy adult. Subjects were then evaluated performing the aforementioned dynamic activities utilizing a ten camera opto-electronic system (Qualisys AB, Gothenburg, Sweden), and three force plates (AMTI, Watertown, MA, USA). Static stance and marker validation was performed using an EOS bi-planar X-ray system (EOS Imaging, Cambridge, MA, USA). Alterations to spine curvature were induced by the means of a custom hyper-tensioned clavicle strap, designed and made with the aid of Spears Prosthetics \& Orthotics/ Rehab Services.

The validation of retro-reflective markers to surgical measurements revealed changes in marker based pelvic tilt differed from changes in radiographic pelvic tilt (difference $\pm 2 \mathrm{SD}$ ) $0 \pm 5 \mathrm{deg}$, changes in marker based spine angles differed from Cobb angles by approximately \pm 10 degs (2SD), and changes in marker based plumbline may under predicted changes in clinical plumbline by $-9 \pm 25 \mathrm{~mm}$. However, it is important to keep in mind that an opto-electronic system was not used in the validation process.

The results of this study suggest that the spine parameters which most influence pelvic tilt are activity dependent, and may be influenced by muscle tightness. During static stance, subjects exhibited a wide range of compensation modes for a hypertensioned clavicle strap, and alterations in TK were the only spinal parameter that correlated with changes in pelvic tilt. Changes in pelvic tilt and hip flexion at peak hip flexion during the activities of Si-St and St-Si significantly correlated with changes in plumbline. During ambulation subjects exhibited two modes of compensation: subjects with clinically tight hamstrings showed an approximately 2:1 ratio of decrease in LL to increase in pelvic tilt, while the remaining subjects demonstrated a 1:0.06 LL to pelvic tilt relationship. 


\section{Limitations and Assumptions}

\section{Instrumental Based}

\section{Limitations}

- Qualisys opto-electric tracking cameras were calibrated to a marker position of less than $\pm 0.6 \mathrm{~mm}$ (mean $=0.4 \mathrm{~mm}$ ). Based on the subjects in this study this translates to a 0.4-0.7 degs uncertainty in LL angles in the sagittal plane.

- Angles derived from marker positions have accuracy of \pm 0.5 deg relative to spinous processes [33].

- AMTI force plates are accurate to $25 \mathrm{~N}$ at full scale output.

- Subjects must stand in EOS system with hands at eye level or on clavicle. Standing area of EOS system is approximately 4 square feet.

- Clavicle straps were not tightened empirically (e.g. tightened to $30 \mathrm{~N}$ )

- Clavicle straps had to be removed during transport to Le Bonheur Children's Hospital.

- Bench is not instrumented, and is of constant height.

\section{Addressed Limitations}

- Clavicle strap position was marked with indelible ink to reproduce change in lordosis. This strategy appears to be successful give the changes seen in the lab were repeated at Le Bonheur Children's Hospital

- Sit-to-Stand and Stand-to-Sit events were evaluated at peak hip flexion.

\section{Assumptions}

- Following calibration procedures, all instruments are within in the manufacturers reported accuracy.

- $\quad$ EOS system derived angle measurements are $\pm 0.5 \mathrm{deg}$.

- Clavicle Strap is tightened equally on both sides.

- Removal and replacement of clavicle strap elicits same response.

\section{Subject Based}

\section{Limitations}

- Subjects limited to young healthy adults.

- Changes in lordosis angle are not constant are subject and activity dependent

- Sagittal lumbar and pelvic measurements during walking have poor within day repeatability, coefficient of multiple correlation values $<0.5$ [47]. Repeatability of peak lumbar and pelvis measures have good within test repeatability (ICC $=0.78$ - 
0.95) during standing pelvic motion activities [28], as due motions of the lumbar spine during sit-to-stand $(\mathrm{ICC}=0.86-0.96)$ [64].

- Subjects were asked to perform activities at comfortable self-selected pace; therefore speed, foot position, head position were not controlled.

- Changes in posture were generally small $<10$ degs.

- Static standing incorporated raised hands and elbows.

\section{Addressed Limitations}

- Subjects were instructed to walk in between trials to randomize foot position.

\section{Assumptions}

- Subject motion is altered solely by lordosis altering device.

- Subjects do not alter their activities because of observation.

\section{Model Based}

\section{Limitations}

- Model is a 6 degree of freedom rigid-link segment model.

- Markers are placed on skin over palpated anatomical landmarks.

- Tracking markers fixed to skin can move independent of anatomical landmarks.

\section{Addressed Limitations}

- Subjects serve as their own controls. Therefore the systematic errors associated with the geometric model are not a factor.

- The same physical therapist and seasoned researcher attached markers.

- The EOS system allows for validation of marker placement, and measurements.

\section{Assumptions}

- Skin motion over the lumbar and pelvis does not influence angle measurements.

\section{Statistical Based}

\section{Limitations}

- Small sample sizes.

- Wilcoxon Signed Rank Test cannot find statistical differences $(\mathrm{p}<0.05)$ with fewer than 5 samples.

- Significance of regressions is heavily influenced by the spread of the data. 


\section{Addressed Limitations}

- Wilcoxon Signed Rank Test was used to determine significance of changes

- No statistics were performed with less than 5 samples.

\section{Assumptions}

- Linear regression slopes can be evaluated using a Student's-t test.

\section{Future Work}

Few investigations have been conducted to determine the functional relationship between the lumbar spine and pelvis. Fewer still have sought to understand these changes in subjects with FSI or flatback deformity. As a result, the possibilities of future investigations are nearly endless. Future studies could range from simply characterizing the repeatability, and replicability, of spine motion during activities of daily living, to studies as elaborate as using bone pins and principle component analysis to determine where and how the spine is compensating.

In the vast possibilities of future work there are several that would build upon and clarify several aspects of this study. One of which would be to further investigate the two modes of compensation observed during the gait activity, as well as to explain the paradoxical increase in pelvic tilt in clinically tight hamstring subjects. With the use of surface and indwelling electromyographic sensors would be possible to monitor the activity of specific erector spinae and hip flexor muscles, as well as hamstrings and abdominal muscles under this altered posture. This information may then be able to explain the different modes of compensation found during gait.

Another valuable extension of this study would be to alter/restrict LL incrementally and consistently, starting at a minimum of \pm 10 deg depending on the study population. By consistently altering the LL it would be possible to solidify predictive trends of compensation, as well as to determine if certain compensations are only present within a range of lordotic conditions. However, it may take some creative engineering or cuing instructions to make this possible.

\section{Conclusion}

The purpose of this study was to determine the functional relationship between the lumbar spine and pelvic tilt during the activities of static standing, ambulation, as well as rising from and descending to a bench. The validation portion of this study suggests that markers may be able to relate changes in pelvic tilt to radiographic pelvic tilt. However, marker based plumbline and spine angles may only give a gross approximation to clinical measures, and future studies are needed to understand the influence of BMI on these measures. The findings in this study suggest: that even relatively small changes in 
spine curvature can alter hip and pelvic position, that different spinal parameters may alter pelvic tilt depending upon the activity being performed, and that hamstring tightness may alter the means which the subject compensates. Future studies should alter/restrict LL incrementally and consistently, as well as include electromyography. 


\section{LIST OF REFERENCES}

1. Rajaee, S.S., et al., Spinal fusion in the United States: analysis of trends from 1998 to 2008. Spine (Phila Pa 1976), 2012. 37(1): p. 67-76.

2. Le Huec, J.C., et al., Sagittal imbalance cascade for simple degenerative spine and consequences: algorithm of decision for appropriate treatment. Eur Spine J, 2011. 20 Suppl 5: p. 699-703.

3. Gottfried, O.N., et al., Spinopelvic parameters in postfusion flatback deformity patients. Spine J, 2009. 9(8): p. 639-47.

4. Acosta, F.L., et al., Changes in coronal and sagittal plane alignment following minimally invasive direct lateral interbody fusion for the treatment of degenerative lumbar disease in adults: a radiographic study. J Neurosurg Spine, 2011. 15(1): p. 92-6.

5. Potter, B.K., L.G. Lenke, and T.R. Kuklo, Prevention and management of iatrogenic flatback deformity. J Bone Joint Surg Am, 2004. 86-A(8): p. 1793-808.

6. Lu, D.C. and D. Chou, Flatback syndrome. Neurosurg Clin N Am, 2007. 18(2): p. 289-94.

7. Cho, K.J., et al., Pedicle Subtraction Osteotomy in Elderly Patients With Degenerative Sagittal Imbalance. Spine (Phila Pa 1976), 2013.

8. Sarwahi, V., et al., Characterization of gait function in patients with postsurgical sagittal (flatback) deformity: a prospective study of 21 patients. Spine (Phila $\mathrm{Pa}$ 1976), 2002. 27(21): p. 2328-37.

9. Blondel, B., et al., Reciprocal sagittal alignment changes after posterior fusion in the setting of adolescent idiopathic scoliosis. Eur Spine J, 2012. 21(10): p. 196471.

10. Aaro, S. and G. Ohlen, The effect of Harrington instrumentation on the sagittal configuration and mobility of the spine in scoliosis. Spine (Phila Pa 1976), 1983. 8(6): p. 570-5.

11. Casey, M.P., et al., The effect of Harrington rod contouring on lumbar lordosis. Spine (Phila Pa 1976), 1987. 12(8): p. 750-3.

12. Lagrone, M.O., et al., Treatment of symptomatic flatback after spinal fusion. J Bone Joint Surg Am, 1988. 70(4): p. 569-80.

13. Kim, K.T., et al., Outcome of pedicle subtraction osteotomies for fixed sagittal imbalance of multiple etiologies: a retrospective review of 140 patients. Spine (Phila Pa 1976), 2012. 37(19): p. 1667-75.

14. Lazennec, J.Y., A. Brusson, and M.A. Rousseau, Lumbar-pelvic-femoral balance on sitting and standing lateral radiographs. Orthop Traumatol Surg Res, 2013. 99(1 Suppl): p. S87-103.

15. Bajwa, N.S., et al., Disk degeneration in lumbar spine precedes osteoarthritic changes in hip. Am J Orthop (Belle Mead NJ), 2013. 42(7): p. 309-12.

16. Prather, H., et al., Impact of coexistent lumbar spine disorders on clinical outcomes and physician charges associated with total hip arthroplasty. Spine J, 2012. 12(5): p. 363-9.

17. Domb, B.G., et al., Comparison of Robotic-assisted and Conventional Acetabular Cup Placement in THA: A Matched-pair Controlled Study. Clin Orthop Relat Res, 2013. 
18. Lazennec, J.Y., et al., Acetabular anteversion with CT in supine, simulated standing, and sitting positions in a THA patient population. Clin Orthop Relat Res, 2011. 469(4): p. 1103-9.

19. Saha, D., et al., The effect of trunk-flexed postures on balance and metabolic energy expenditure during standing. Spine (Phila Pa 1976), 2007. 32(15): p. 1605-11.

20. Eilander, W., et al., Functional acetabular component position with supine total hip replacement. Bone Joint J, 2013. 95-B(10): p. 1326-1331.

21. Vincent, K.R., et al., The pathophysiology of osteoarthritis: a mechanical perspective on the knee joint. PM R, 2012. 4(5 Suppl): p. S3-9.

22. Beaupre, G.S., S.S. Stevens, and D.R. Carter, Mechanobiology in the development, maintenance, and degeneration of articular cartilage. J Rehabil Res Dev, 2000. 37(2): p. 145-51.

23. Dandachli, W., et al., The influence of pelvic tilt on acetabular orientation and cover: a three-dimensional computerised tomography analysis. Hip Int, 2013. 23(1): p. 87-92.

24. Lee, C.S., et al., Dynamic sagittal imbalance of the spine in degenerative flat back: significance of pelvic tilt in surgical treatment. Spine (Phila Pa 1976), 2001. 26(18): p. 2029-35.

25. Oken, F., et al., Short or long fusion after thoracolumbar burst fractures does not alter selected gait parameters: a preliminary study. J Orthop Res, 2011. 29(6): p. 915-8.

26. Lafage, V., et al., Spino-pelvic parameters after surgery can be predicted: a preliminary formula and validation of standing alignment. Spine (Phila $\mathrm{Pa} 1976$ ), 2011. 36(13): p. 1037-45.

27. Schwab, F., et al., Adult spinal deformity-postoperative standing imbalance: how much can you tolerate? An overview of key parameters in assessing alignment and planning corrective surgery. Spine (Phila Pa 1976), 2010. 35(25): p. 2224-31.

28. Levine, D. and M.W. Whittle, The effects of pelvic movement on lumbar lordosis in the standing position. J Orthop Sports Phys Ther, 1996. 24(3): p. 130-5.

29. Preece, S.J., et al., Variation in pelvic morphology may prevent the identification of anterior pelvic tilt. J Man Manip Ther, 2008. 16(2): p. 113-7.

30. Lund, P.J., E.A. Krupinski, and W.J. Brooks, Ultrasound evaluation of sacroiliac motion in normal volunteers. Acad Radiol, 1996. 3(3): p. 192-6.

31. Boulay, C., et al., Sagittal alignment of spine and pelvis regulated by pelvic incidence: standard values and prediction of lordosis. Eur Spine J, 2006. 15(4): p. 415-22.

32. Roussouly, P., et al., Classification of the normal variation in the sagittal alignment of the human lumbar spine and pelvis in the standing position. Spine (Phila Pa 1976), 2005. 30(3): p. 346-53.

33. Hashemirad, F., et al., Validity and reliability of skin markers for measurement of intersegmental mobility at L2-3 and L3-4 during lateral bending in healthy individuals: a fluoroscopy study. J Bodyw Mov Ther, 2013. 17(1): p. 46-52.

34. Morl, F. and R. Blickhan, Three-dimensional relation of skin markers to lumbar vertebrae of healthy subjects in different postures measured by open MRI. Eur Spine J, 2006. 15(6): p. 742-51. 
35. Whittle, M.W. and D. Levine, Measurement of lumbar lordosis as a component of clinical gait analysis. Gait Posture, 1997(5): p. 101-107.

36. Gottipati, P., et al., Crouch gait in persons with positive sagittal spine alignment resolves with surgery. Gait Posture, 2014. 39(1): p. 372-7.

37. Whittle, M.W. and D. Levine, Three-dimensional relationships between the movements of the pelvis and lumbar spine during normal gait. Human Movement Science 1999(18): p. 681-92.

38. Fowler, N.E., A.L. Rodacki, and C.D. Rodacki, Changes in stature and spine kinematics during a loaded walking task. Gait Posture, 2006. 23(2): p. 133-41.

39. Syczewska, M., T. Oberg, and D. Karlsson, Segmental movements of the spine during treadmill walking with normal speed. Clin Biomech (Bristol, Avon), 1999. 14(6): p. 384-8.

40. Bell, A.L., D.R. Pedersen, and R.A. Brand, A comparison of the accuracy of several hip center location prediction methods. J Biomech, 1990. 23(6): p. 61721.

41. Bell, A.L., R.A. Brand, and D.R. Pedersen, Prediction of hip joint centre location from external landmarks. Human Movement Science, 1989. 8(1): p. 3-16.

42. Kuo, M.Y., et al., Influence of soft tissue artifacts on the calculated kinematics and kinetics of total knee replacements during sit-to-stand. Gait Posture, 2011. 33(3): p. 379-84.

43. Richards, C. and J.S. Higginson, Knee contact force in subjects with symmetrical OA grades: differences between OA severities. J Biomech, 2010. 43(13): p. 2595600.

44. Gille, O., et al., Reliability of $3 D$ reconstruction of the spine of mild scoliotic patients. Spine (Phila Pa 1976), 2007. 32(5): p. 568-73.

45. Pomero, V., et al., Fast accurate stereoradiographic 3D-reconstruction of the spine using a combined geometric and statistic model. Clin Biomech (Bristol, Avon), 2004. 19(3): p. 240-7.

46. Fotoohabadi, M.R., E.A. Tully, and M.P. Galea, Kinematics of rising from a chair: image-based analysis of the sagittal hip-spine movement pattern in elderly people who are healthy. Phys Ther, 2010. 90(4): p. 561-71.

47. Chan, P.Y., H.K. Wong, and J.C. Goh, The repeatablity of spinal motion of normal and scoliotic adolescents during walking. Gait Posture, 2006. 24(2): p. 219-28.

48. Schindelin, J., et al., Fiji: an open-source platform for biological-image analysis. Nat Methods, 2012. 9(7): p. 676-82.

49. Lafage, V., et al., Pelvic tilt and truncal inclination: two key radiographic parameters in the setting of adults with spinal deformity. Spine (Phila Pa 1976), 2009. 34(17): p. E599-606.

50. Shum, G.L., J. Crosbie, and R.Y. Lee, Three-dimensional kinetics of the lumbar spine and hips in low back pain patients during sit-to-stand and stand-to-sit. Spine (Phila Pa 1976), 2007. 32(7): p. E211-9.

51. Shum, G.L., J. Crosbie, and R.Y. Lee, Effect of low back pain on the kinematics and joint coordination of the lumbar spine and hip during sit-to-stand and standto-sit. Spine (Phila Pa 1976), 2005. 30(17): p. 1998-2004. 
52. Rose, J., et al., Energy expenditure index of walking for normal children and for children with cerebral palsy. Dev Med Child Neurol, 1990. 32(4): p. 333-40.

53. Lloyd-Roberts, G.C., A.M. Jackson, and J.S. Albert, Avulsion of the distal pole of the patella in cerebral palsy. A cause of deteriorating gait. J Bone Joint Surg Br, 1985. 67(2): p. 252-4.

54. MacWilliams, B.A., et al., Assessment of three-dimensional lumbar spine vertebral motion during gait with use of indwelling bone pins. J Bone Joint Surg Am, 2013. 95(23): p. e1841-8.

55. Needham, R., R. Naemi, and N. Chockalingam, Quantifying lumbar-pelvis coordination during gait using a modified vector coding technique. J Biomech, 2014. 47(5): p. 1020-6.

56. Gracovetsky, S., An hypothesis for the role of the spine in human locomotion: a challenge to current thinking. J Biomed Eng, 1985. 7(3): p. 205-16.

57. Stanhope, S.J., et al., Kinematic-based technique for event time determination during gait. Med Biol Eng Comput, 1990. 28(4): p. 355-60.

58. Borman, N.P., E. Trudelle-Jackson, and S.S. Smith, Effect of stretch positions on hamstring muscle length, lumbar flexion range of motion, and lumbar curvature in healthy adults. Physiother Theory Pract, 2011. 27(2): p. 146-54.

59. World Health Organization, W.H.O., International Classification of Impairments, Disabilities, and Handicaps: A manual of classification relating to the consequences of disease. 1980, World Health Organization: Geneva, Switzerland.

60. Dall, P.M. and A. Kerr, Frequency of the sit to stand task: An observational study of free-living adults. Appl Ergon, 2010. 41(1): p. 58-61.

61. Eriksrud, O. and R.W. Bohannon, Relationship of knee extension force to independence in sit-to-stand performance in patients receiving acute rehabilitation. Phys Ther, 2003. 83(6): p. 544-51.

62. Janssen, W.G., H.B. Bussmann, and H.J. Stam, Determinants of the sit-to-stand movement: a review. Phys Ther, 2002. 82(9): p. 866-79.

63. Tully, E.A., M.R. Fotoohabadi, and M.P. Galea, Sagittal spine and lower limb movement during sit-to-stand in healthy young subjects. Gait Posture, 2005. 22(4): p. 338-45.

64. Parkinson, S., et al., Upper and lower lumbar segments move differently during sit-to-stand. Man Ther, 2013. 18(5): p. 390-4.

65. Kuo, Y.L., E.A. Tully, and M.P. Galea, Kinematics of sagittal spine and lower limb movement in healthy older adults during sit-to-stand from two seat heights. Spine (Phila Pa 1976), 2010. 35(1): p. E1-7.

66. Cacciatore, T.W., et al., Prolonged weight-shift and altered spinal coordination during sit-to-stand in practitioners of the Alexander Technique. Gait Posture, 2011. 34(4): p. 496-501.

67. Kralj, A., R.J. Jaeger, and M. Munih, Analysis of standing up and sitting down in humans: definitions and normative data presentation. J Biomech, 1990. 23(11): p. 1123-38.

68. Cheynel, N., et al., [Standing-up/sitting-down movement. Electromyographic analysis of 4 muscles of lower limb and the erector spinae muscle: study of anticipatory postural adjustments]. Morphologie, 2002. 86(274): p. 23-6. 
69. Lamontagne, M., et al., Lower-limb joint mechanics after total hip arthroplasty during sitting and standing tasks. J Orthop Res, 2012. 30(10): p. 1611-7.

70. Smith, T., et al., What activities cause hip dislocation? A review of 100 total hip replacement dislocations. Advances in Physiotherapy, 2012. 14: p. 55-60.

71. Su, F.C., K.A. Lai, and W.H. Hong, Rising from chair after total knee arthroplasty. Clin Biomech (Bristol, Avon), 1998. 13(3): p. 176-181.

72. Spyropoulos, G., et al., Biomechanics of sit-to-stand transition after muscle damage. Gait Posture, 2013. 38(1): p. 62-7. 


\section{APPENDIX A. TECHNICAL INSTRUMENT SPECIFICATIONS}

\begin{tabular}{|c|c|c|c|c|c|c|c|c|}
\hline \multicolumn{2}{|l|}{ Dimensions (WxlxH) } & \multicolumn{2}{|c|}{$464 \times 508 \times 83 \mathrm{~mm}$} & \multicolumn{3}{|c|}{ Mounting hardware } & \multicolumn{2}{|c|}{ Recommended } \\
\hline Weight & & \multicolumn{2}{|l|}{$28.18 \mathrm{~kg}$} & \multicolumn{3}{|c|}{ Sensing elements } & \multicolumn{2}{|c|}{ Strain gage bridge } \\
\hline Channels & & \multicolumn{2}{|c|}{$\mathrm{Fx}, \mathrm{Fy}, \mathrm{Fz}, \mathrm{Mx}, \mathrm{My}, \mathrm{Mz}$} & \multicolumn{3}{|c|}{ Amplifier } & \multicolumn{2}{|c|}{ Required } \\
\hline Top plate material & & \multicolumn{2}{|l|}{ Aluminum } & \multicolumn{3}{|c|}{ Analog outputs } & \multicolumn{2}{|c|}{6 Channels } \\
\hline Temperature range & & \multicolumn{2}{|c|}{-17.78 to $51.67^{\circ} \mathrm{C}$} & \multicolumn{3}{|c|}{ Digital outputs } & \multicolumn{2}{|l|}{ None } \\
\hline Excitation & & \multicolumn{2}{|c|}{$10 \mathrm{~V}$ maximum } & \multicolumn{3}{|c|}{ Crosstalk } & \multicolumn{2}{|c|}{$<2 \%$ on all channels } \\
\hline Fx, Fy, Fz hysteresis & & \multicolumn{2}{|c|}{ $\pm 0.2 \%$ full scale output } & \multicolumn{3}{|c|}{ Fx, Fy, Fz non-linearity } & \multicolumn{2}{|c|}{ $\pm 0.2 \%$ full scale output } \\
\hline Channel & Fx & Fy & $\mathrm{Fz}$ & Units & $M x$ & My & $\mathrm{Mz}$ & Units \\
\hline Capacity & 4448 & 4448 & 8896 & $\mathrm{~N}$ & 2258 & 2258 & 1129 & $\mathrm{~N}-\mathrm{m}$ \\
\hline Sensitivity & 0.337 & 0.337 & 0.0843 & $\mu \vee / v-N$ & 0.797 & 0.797 & 1.68 & $\mu v / v-N-m$ \\
\hline Natural frequency & 360 & 360 & 500 & $\mathrm{~Hz}$ & - & - & - & $\mathrm{Hz}$ \\
\hline
\end{tabular}

\section{Figure A-1: $\quad$ AMTI OR6-7-2000 Forceplate Technical Specifications}

Source: Reprinted with kind permission from Advanced Mechanical Technology, Inc.: http://www.amti.biz/AMTIpibrowser.aspx?_VIEWSTATE=\%2FwEPDwUKMTgyNjIx Njg1OGRk\&iListbox $1=350 \&$ iListbox2=378\&iListbox3=OR6-

$7 \&$ iUnits $=\&$ iNewpageURL $=\&$ iScrollTop $=0 \& i$ ScrollTop2 $=0 \& i$ ScrollTop3=30\&iArrIma ge_URL $=. \% 2$ FImage $\% 2520$ file $\% 2$ Fprogress_1.gif $\% 2$ C. $\% 2$ FImage $\% 2520$ file $\% 2$ Fprogre ss_2.gif $\% 2$ C. $\% 2$ FImage $\% 2520$ file $\% 2$ Fprogress_3.gif $\% 2$ C. $\% 2$ FImage $\% 2520$ file $\% 2$ Fpro gress_4.gif $\% 2$ C.\%2FImage $\% 2520$ file $\% 2$ Fprogress_5.gif $\% 2$ C. $\% 2$ FImage $\% 2520$ file $\% 2 \mathrm{~F}$ progress_6.gif\%2C.\%2FImage $\% 2520$ file $\% 2$ Fprogress_7.gif $\% 2$ C. $\% 2$ FImage $\% 2520$ file $\%$ 2Fprogress_8.gif\%2C.\%2FImage $\% 2520$ file $\% 2$ Fprogress_9.gif accessed 4-30-2014 


\section{APPENDIX B. ADDITIONAL GRAPHS}

\section{Additional "Validation of Motion Analysis Spinal and Pelvic Measures Using an EOS X-ray System" Graphs}

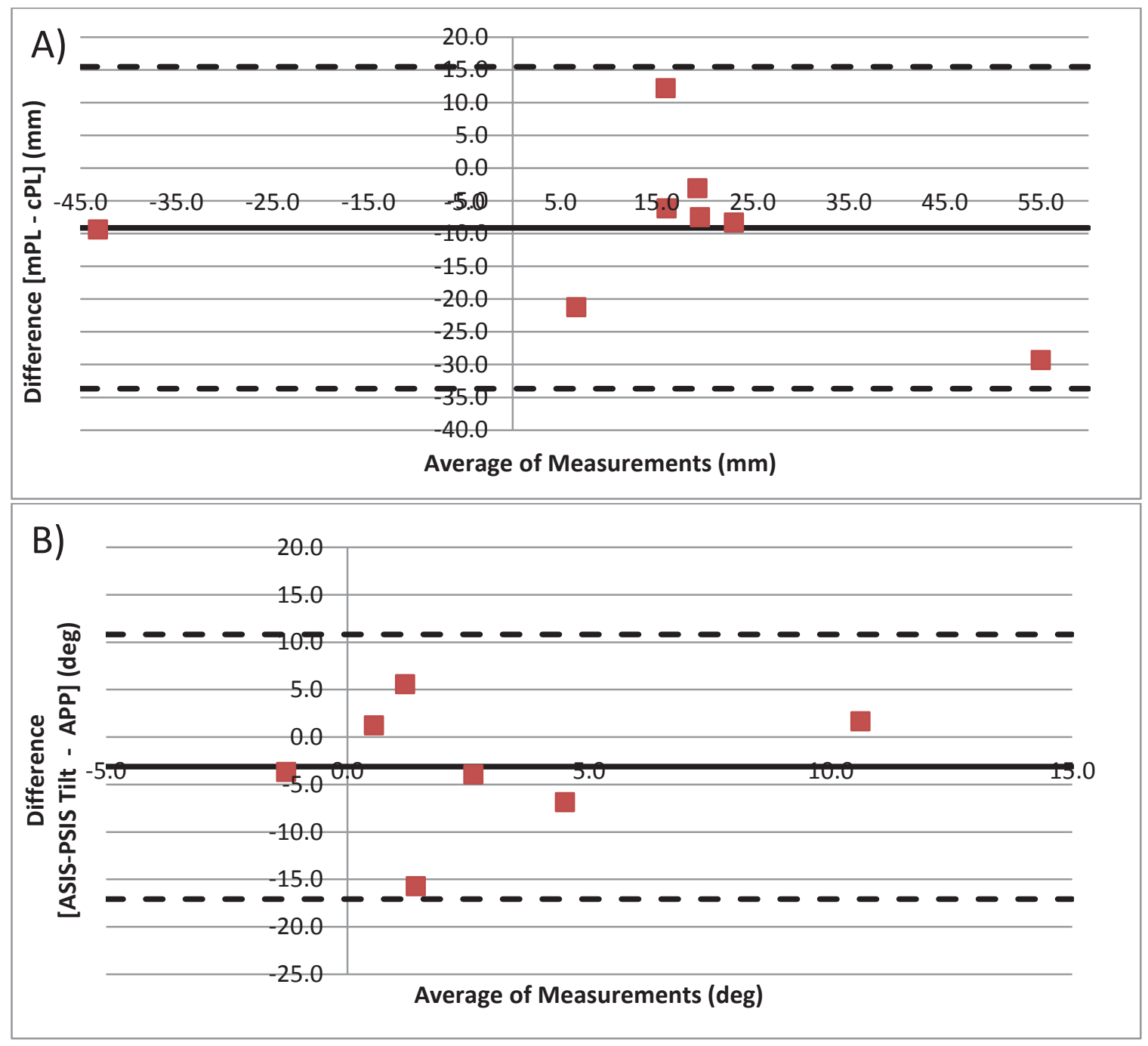

Figure B-1: Bland-Altman Plots of Changes in Plumbline and Clinical Pelvic Tilt Measures

A) Bland-Altman of plot of changes in Marker vs Clinical Plumbline B) Bland-Altman Plot of changes in ASIS-PSIS tilt vs APP.

Solid black line represents mean difference between measurements

Dashed lines represent the mean $\pm 2 * \mathrm{SD}$ 


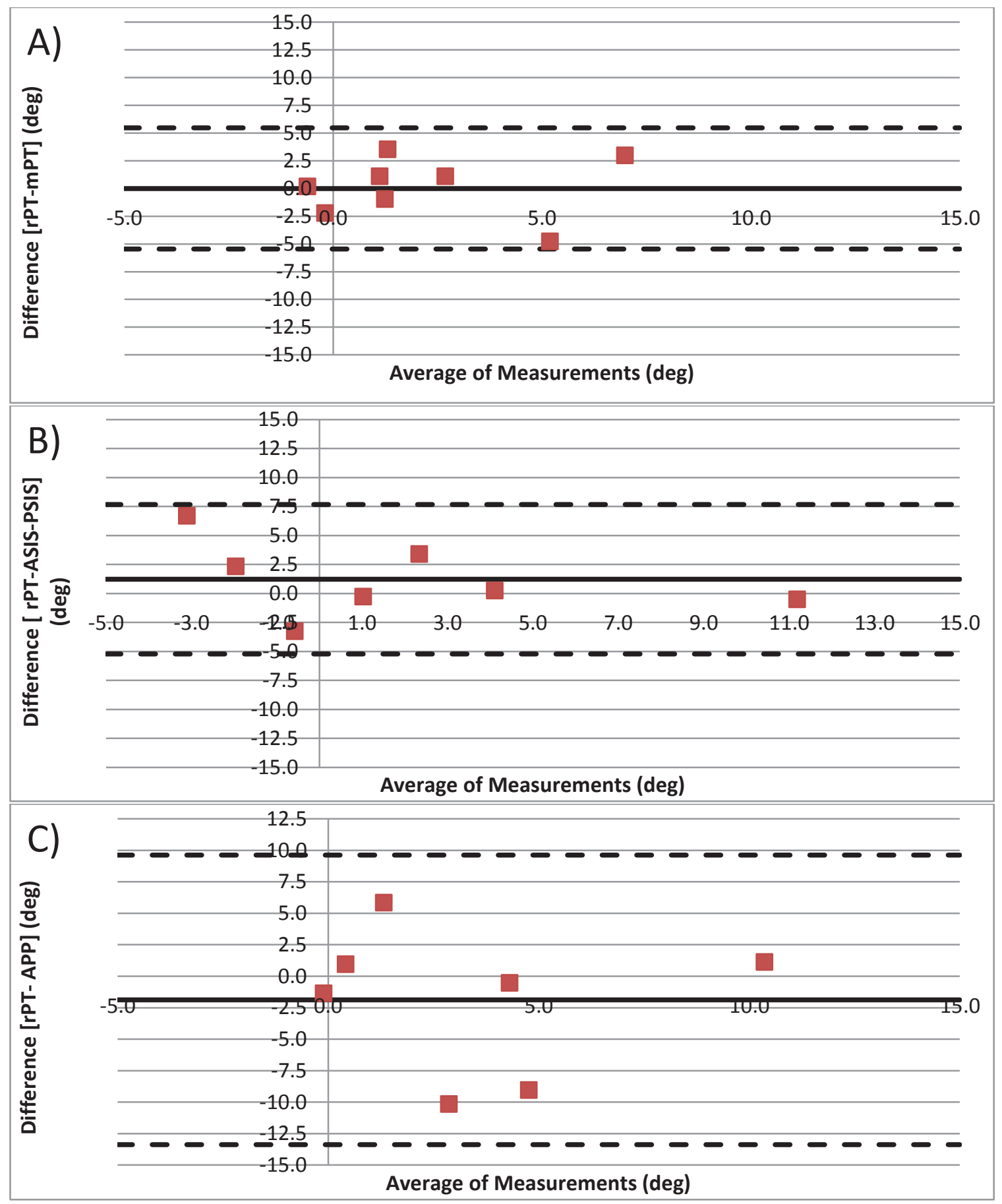

Figure B-2: Bland-Altman Plots of and Radiographic vs Pelvic Tilt Measures

A) Bland-Altman of plot of changes in Radiographic Pelvic Tilt vs Marker Pelvic Tilt B) Bland-Altman Plot of in Radiographic Pelvic Tilt vs ASIS-PSIS Tilt. C) Bland-Altman of plot of changes in Radiographic Pelvic Tilt vs APP

Solid black line represents mean difference between measurements

Dashed lines represent the mean $\pm 2 * \mathrm{SD}$ 
Additional "EOS Bi-planar X-ray Validates Hyper-Tensioned Clavicle Strap Alters Lumbar Lordosis in Standing” Graphs

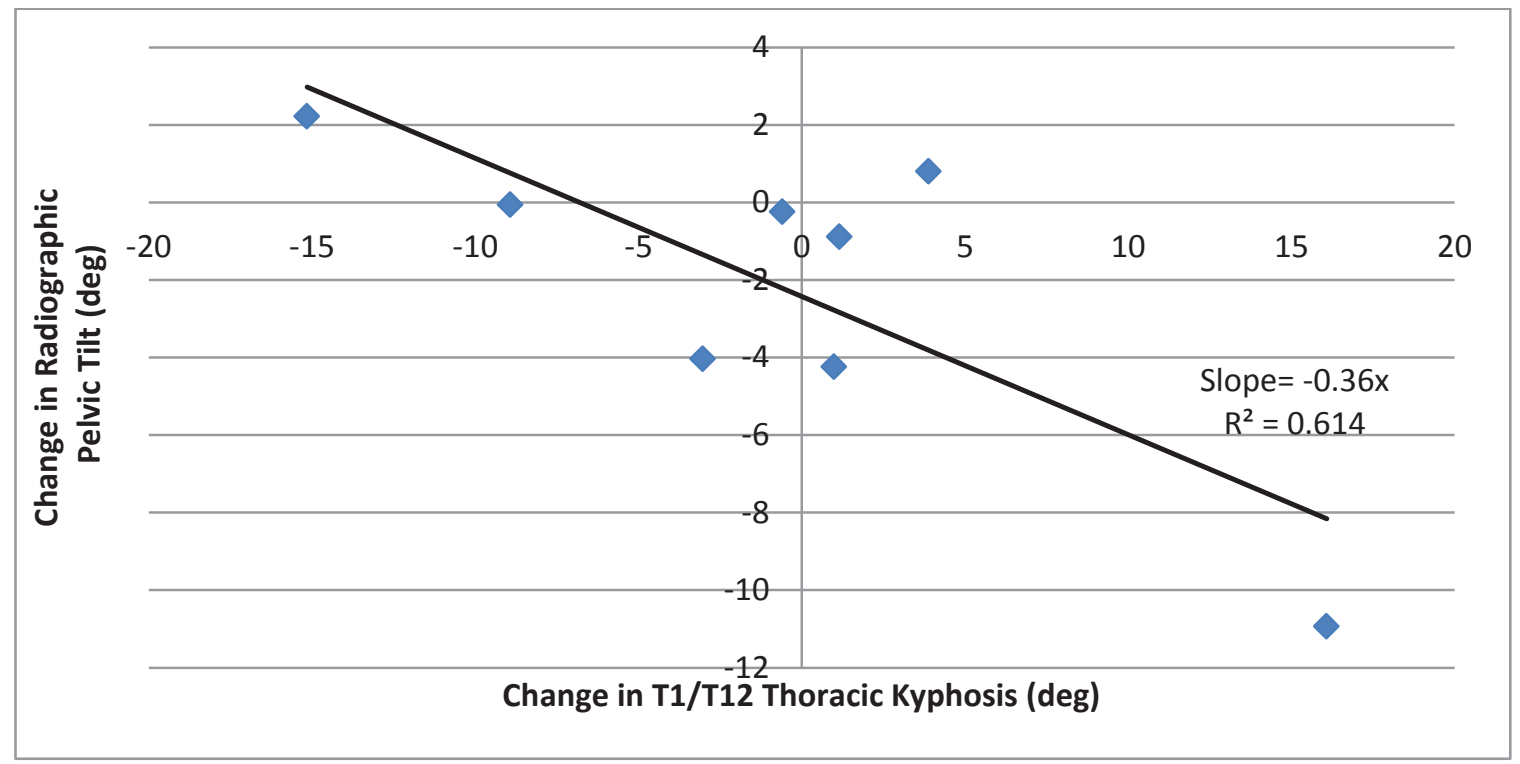

Figure B-3: Linear Regression of Change in Radiographic Pelvic Tilt vs Change in T1/T12 Cobb Angle 
Additional "Spino-Pelvic Kinematics of Gait under Different Sagittal Alignments" Graphs

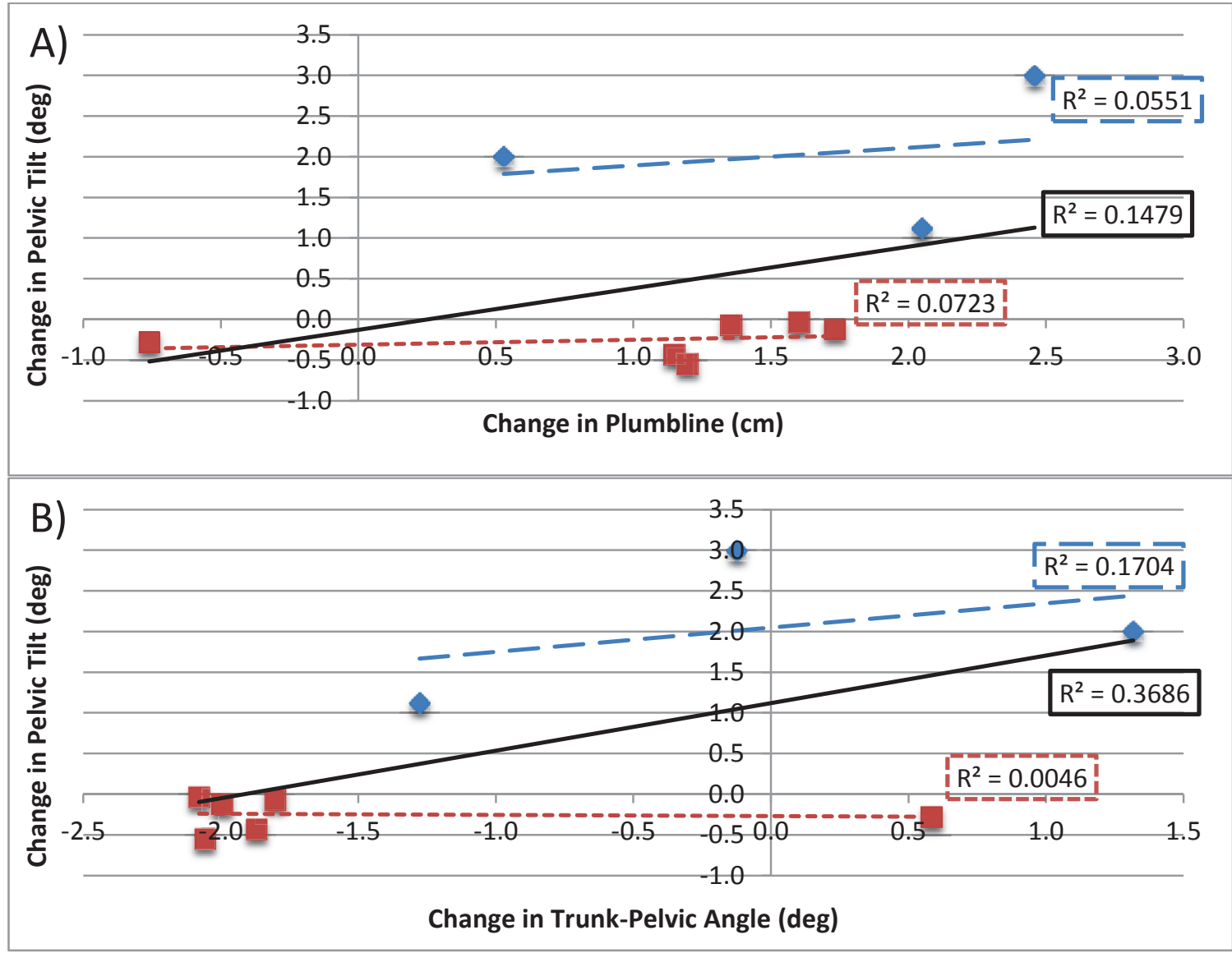

Figure B-4: Change in Pelvic Tilt Correlations during Gait Correlations

A) Linear Regressions of Change in Pelvic Tilt vs Change in Plumbline B) Linear Regressions of Change in Pelvic Tilt vs Change in Trunk-Pelvic Angle

Red squares represent the Compensation 1 group. Blue diamonds represent the Compensation 2 group. The lines represent linear regressions for their respective groups: blue large dashed line $=$ red short dashed $=$ Compensation 1 , Compensation 2 , and solid black $=$ Overall. 


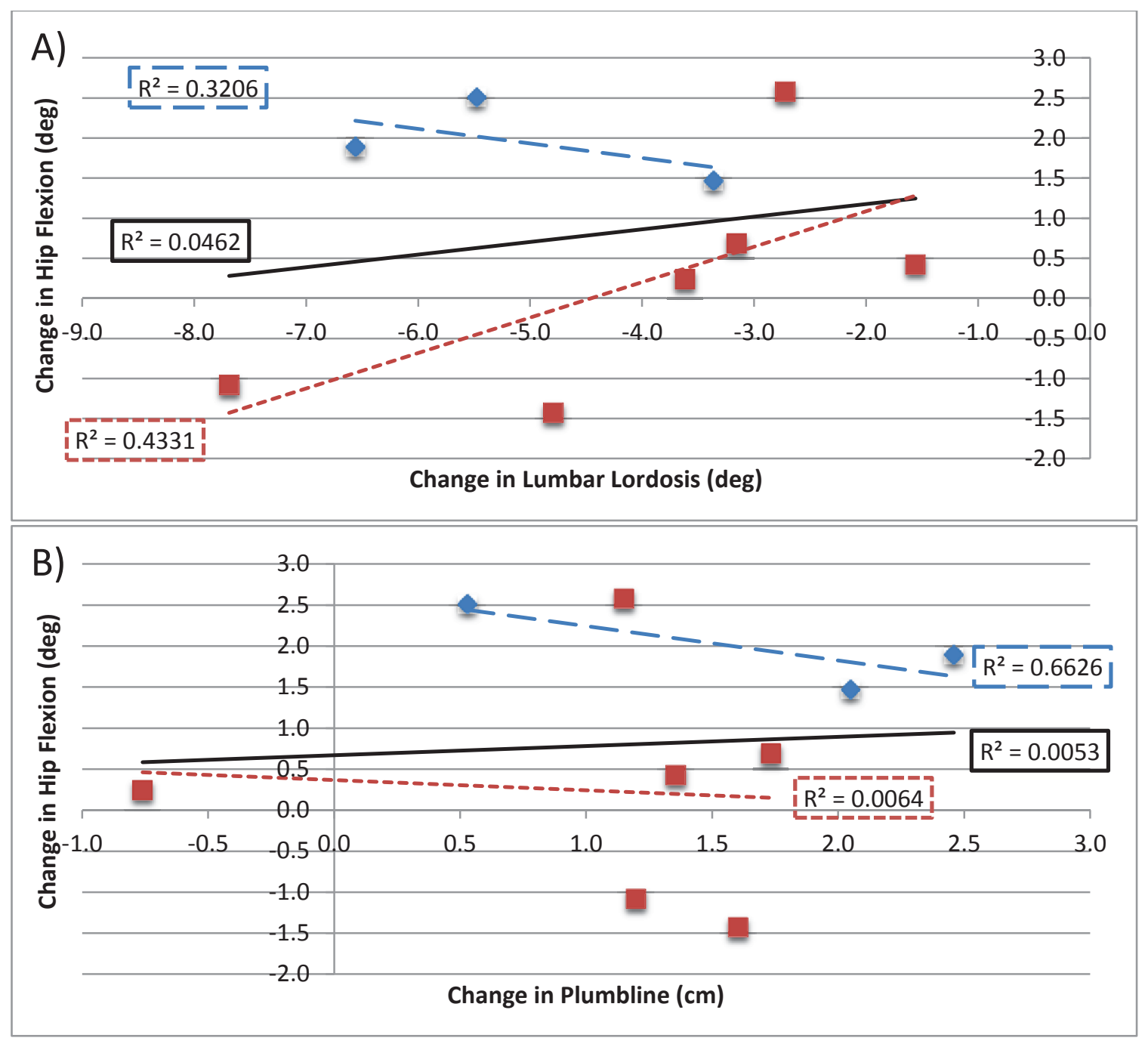

Figure B-5: Change in Hip Flexion Correlations during Gait

A) Linear Regressions of Change in Hip Flexion vs Change in Lumbar Lordosis B) Linear Regressions of Change in Hip Flexion vs Change in Plumbline

Red squares represent the Compensation 1 group. Blue diamonds represent the Compensation 2 group. The lines represent linear regressions for their respective groups: blue large dashed line $=$ red short dashed $=$ Compensation 1 , Compensation 2 , and solid black $=$ Overall. 


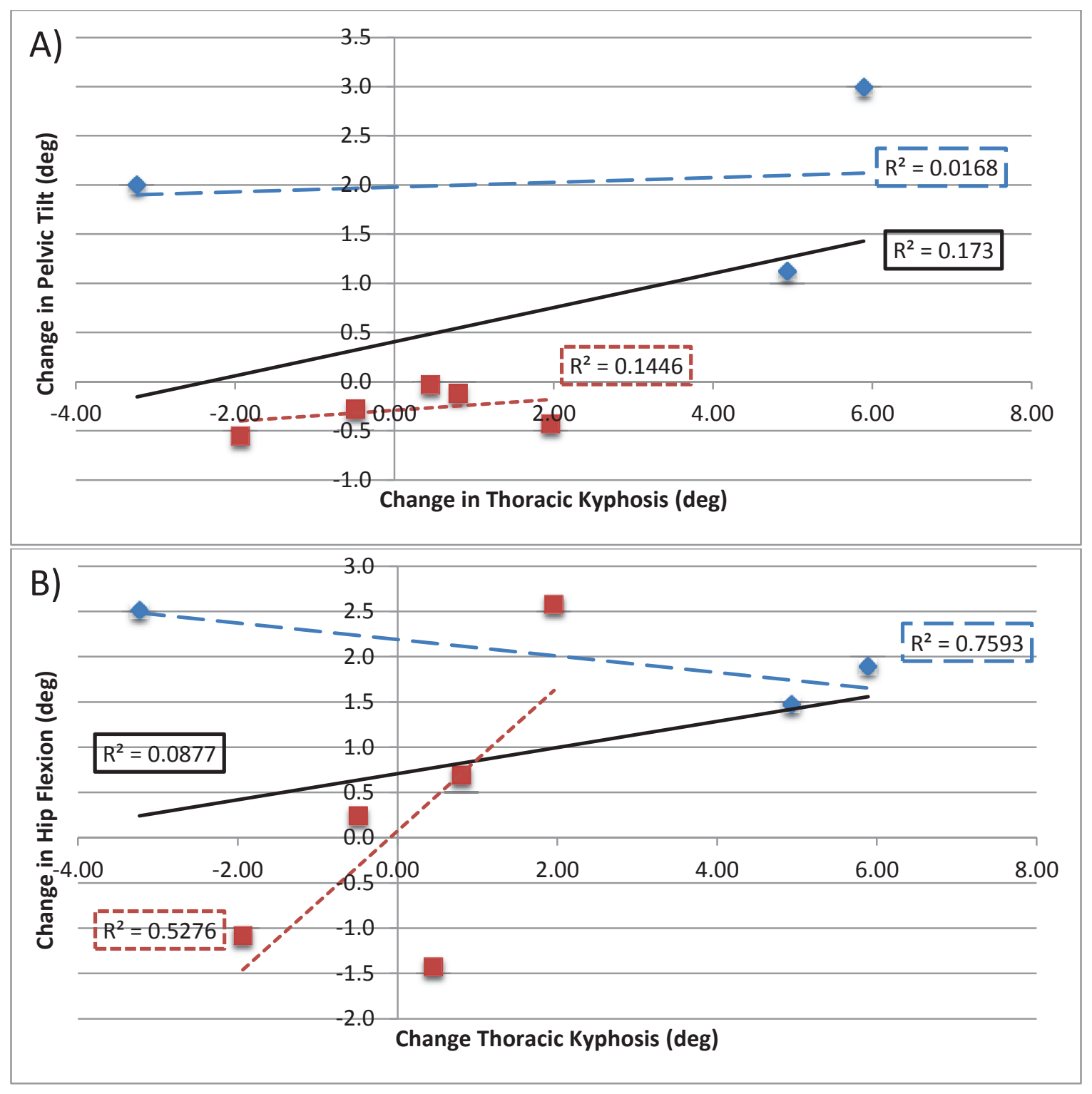

Figure B-6: Change in Pelvic Tilt and Hip Flexion vs Thoracic Kyphosis Correlations

A) Linear Regressions of Change in Pelvic Tilt vs Change in Thoracic Kyphosis B) Linear Regressions of Change in Hip Flexion vs Change in Thoracic Kyphosis Red squares represent the Compensation 1 group. Blue diamonds represent the Compensation 2 group. The lines represent linear regressions for their respective groups: blue large dashed line $=$ red short dashed $=$ Compensation 1 , Compensation 2 , and solid black $=$ Overall. 
Additional "Spino-Pelvic Kinematics of Sit-to-Stand and Stand-to-Sit Activities under Different Sagittal Alignments" Graphs

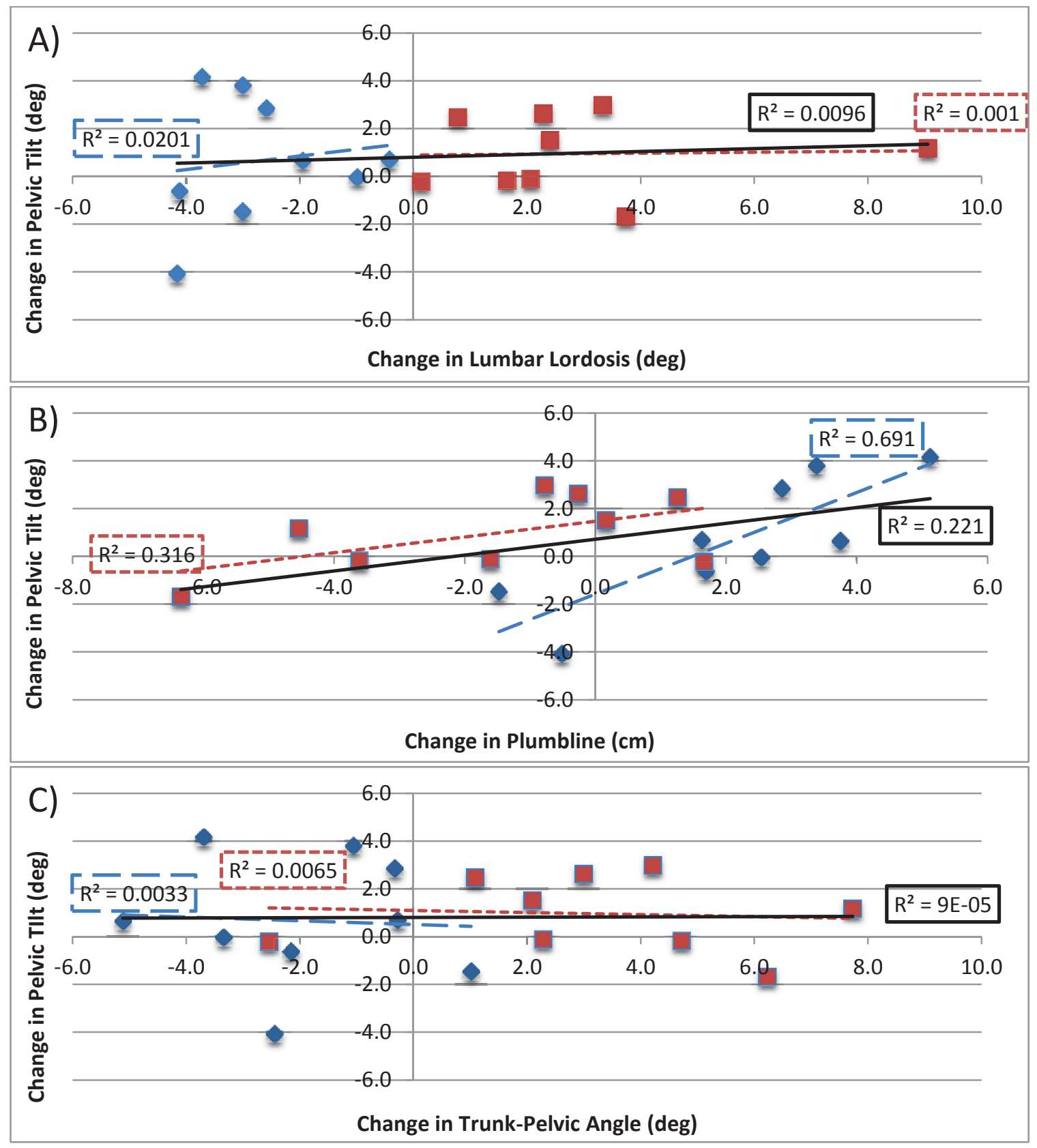

Figure B-7: $\quad$ Stand-to-Sit Change in Pelvic Tilt Correlations at Peak Hip Flexion A) Linear Regressions of Change in Pelvic Tilt vs Change in Lumbar Lordosis B) Linear Regressions of Change in Pelvic Tilt vs Change in Plumbline C) Linear Regressions of Change in Pelvic Tilt vs Change in Trunk-Pelvic Angle

Blue diamonds represent the Decreased lumbar lordosis group. Red squares represent the Increased in lumbar lordosis group. The lines represent linear regressions for their respective groups: blue large dashed line $=$ Decreased, red short dashed $=$ Increased, and solid black $=$ Overall. 


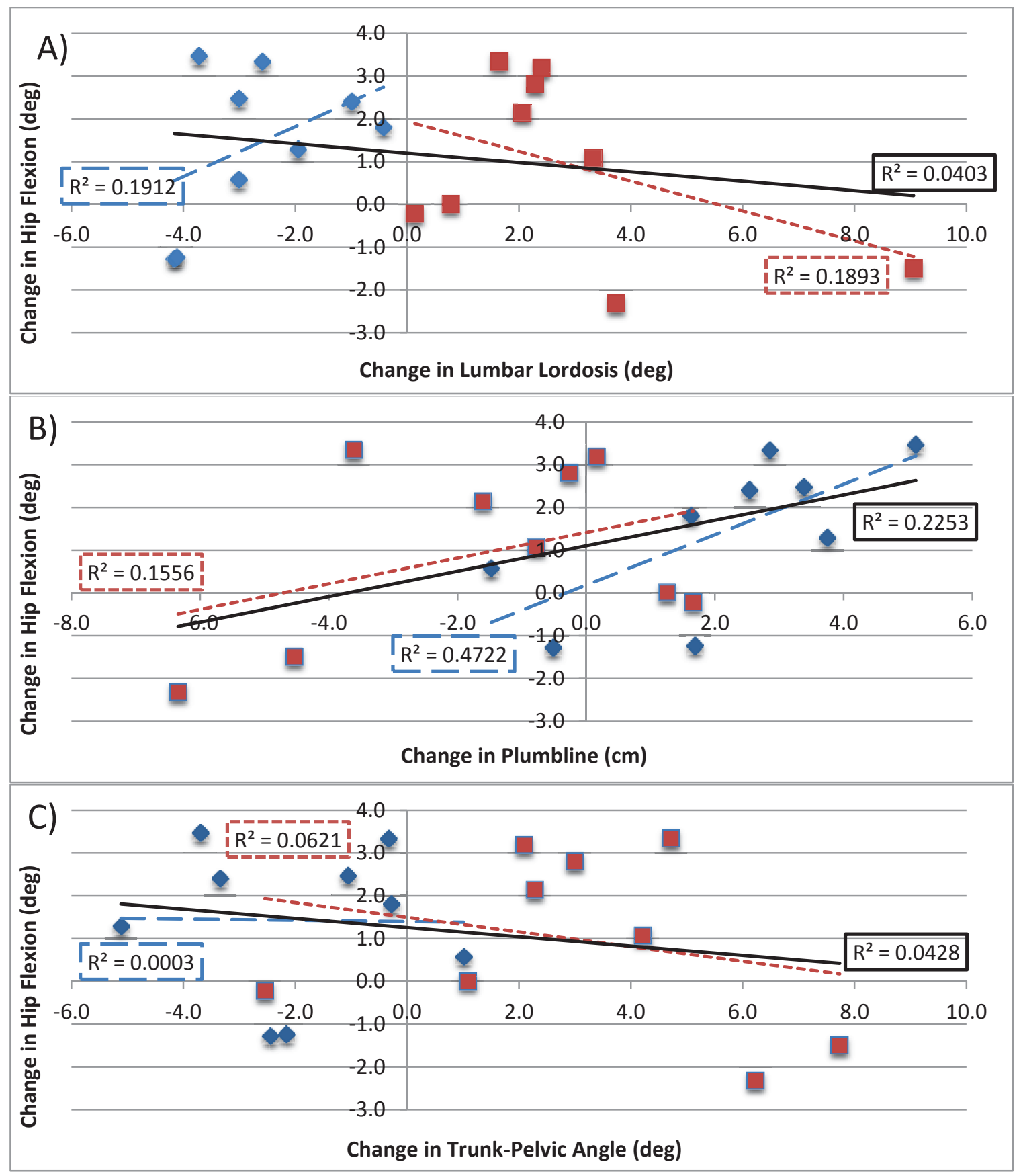

Figure B-8: $\quad$ Stand-to-Sit Changes in Hip Flexion Correlations at Peak Hip Flexion

A) Linear Regressions of Change in Hip Flexion vs Change in Lumbar Lordosis B) Linear Regressions of Change in Hip Flexion vs Change in Plumbline C) Linear Regressions of Change in Hip Flexion vs Change in Trunk-Pelvic Angle Blue diamonds represent the Decreased lumbar lordosis group. Red squares represent the Increased in lumbar lordosis group. The lines represent linear regressions for their respective groups: blue large dashed line $=$ Decreased, red short dashed $=$ Increased, and solid black $=$ Overall. 


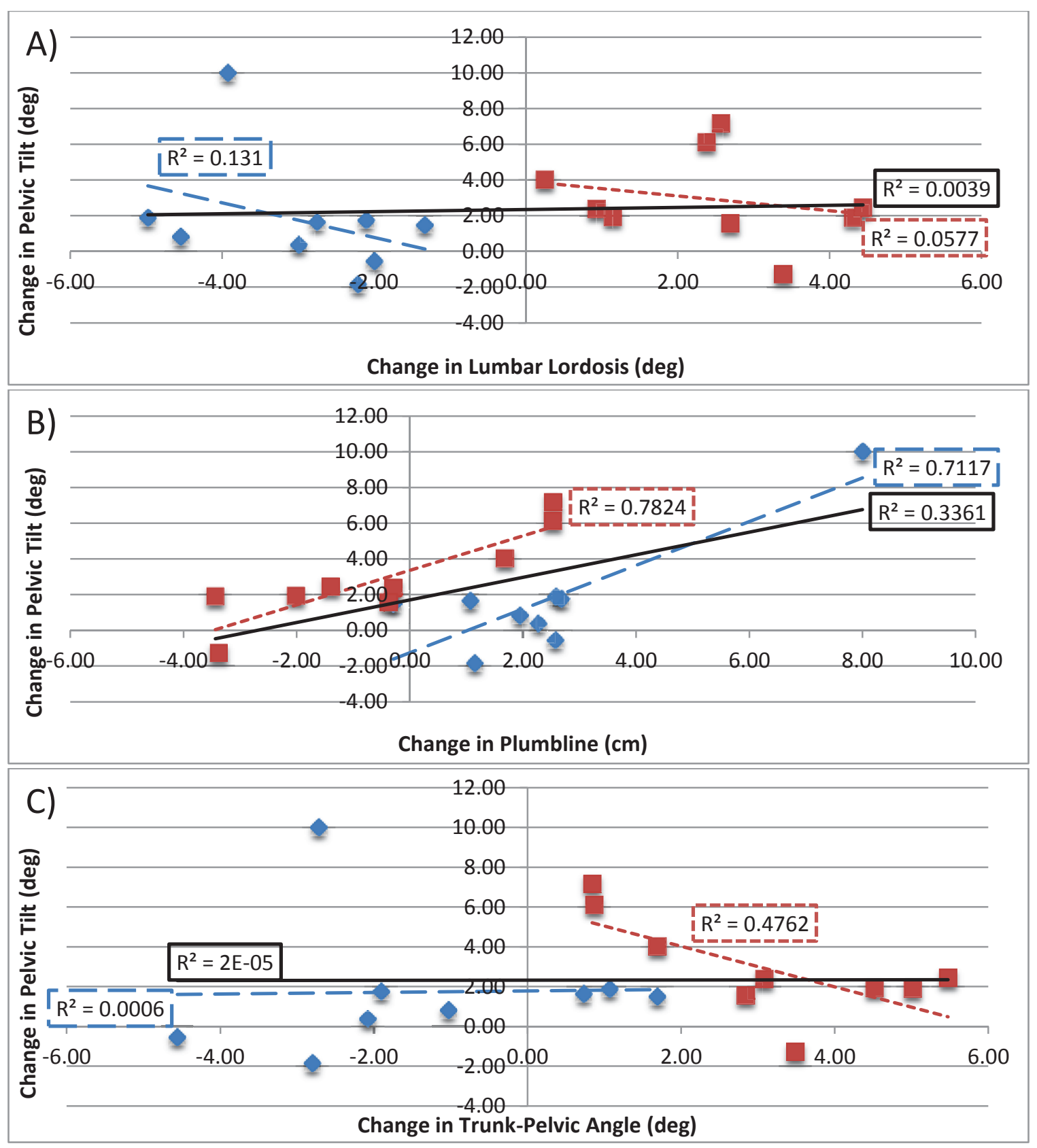

Figure B-9: $\quad$ Sit-to-Stand Changes in Pelvic Tilt Correlations at Peak Hip Flexion A) Linear Regressions of Change in Pelvic Tilt vs Change in Lumbar Lordosis B) Linear Regressions of Change in Pelvic Tilt vs Change in Plumbline C) Linear Regressions of Change in Pelvic Tilt vs Change in Trunk-Pelvic Angle

Blue diamonds represent the Decreased lumbar lordosis group. Red squares represent the Increased in lumbar lordosis group. The lines represent linear regressions for their respective groups: blue large dashed line $=$ Decreased, red short dashed $=$ Increased, and solid black $=$ Overall. 


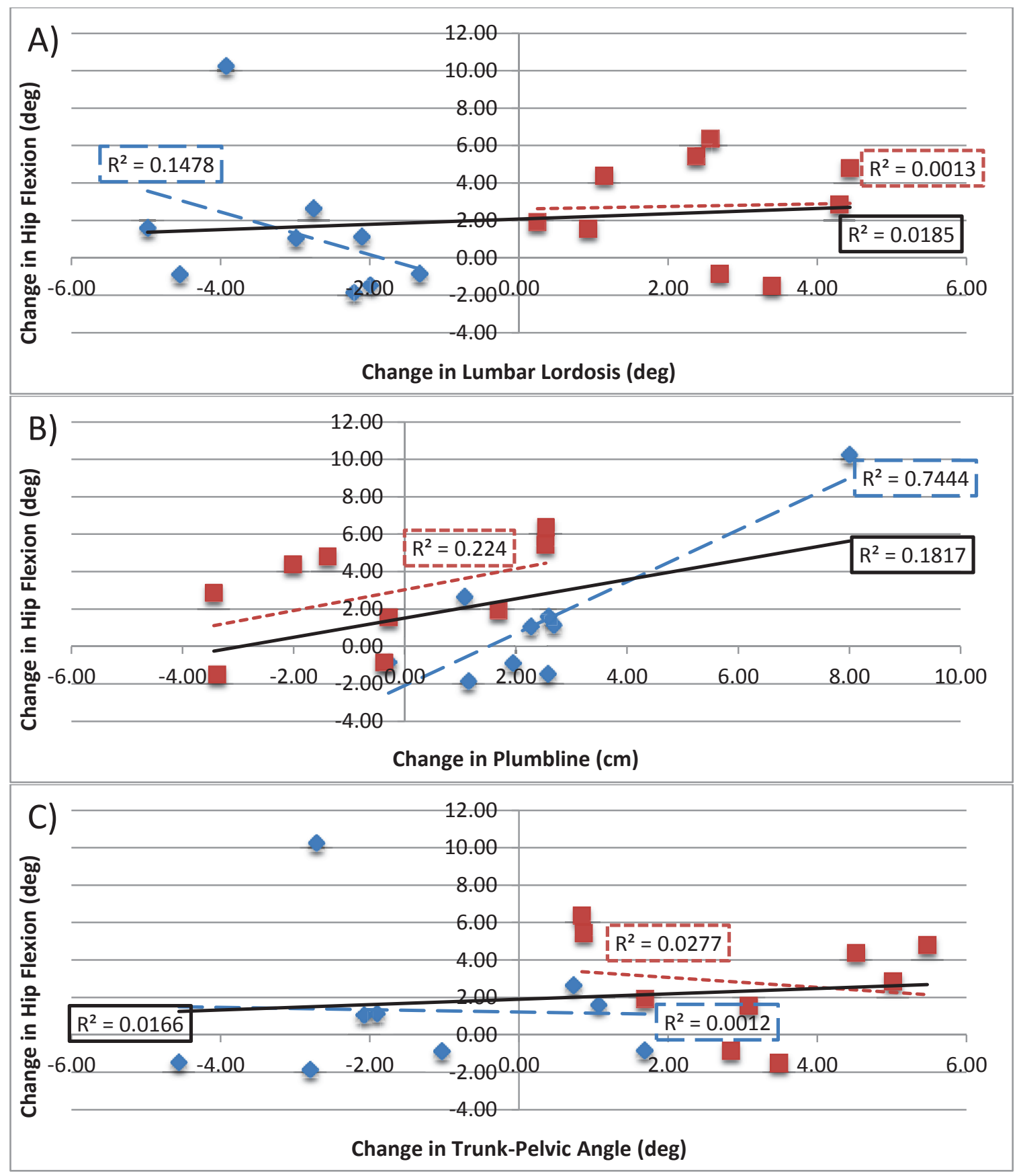

Figure B-10: Sit-to-Stand Changes in Hip Flexion Correlations at Peak Hip Flexion

A) Linear Regressions of Change in Hip Flexion vs Change in Lumbar Lordosis B) Linear Regressions of Change in Hip Flexion vs Change in Plumbline C) Linear Regressions of Change in Hip Flexion vs Change in Trunk-Pelvic Angle Blue diamonds represent the Decreased lumbar lordosis group. Red squares represent the Increased in lumbar lordosis group. The lines represent linear regressions for their respective groups: blue large dashed line $=$ Decreased, red short dashed $=$ Increased, and solid black $=$ Overall. 
APPENDIX C. NORMATIVE DATA TABLES

Table 1. Normative Radiographic Spinopelvic Values

\begin{tabular}{|c|c|c|c|c|c|c|}
\hline \multirow[b]{2}{*}{ Parameter } & \multicolumn{6}{|c|}{ Normative Values and References } \\
\hline & $\begin{array}{c}\text { Schwab et al, } \\
2006^{9}\end{array}$ & $\begin{array}{l}\text { Berthonnaud } e t \text { al, } \\
\qquad 2005^{4}\end{array}$ & $\begin{array}{l}\text { Vialle et al, } \\
2005^{15}\end{array}$ & $\begin{array}{c}\text { Legaye et al, } \\
1998^{13}\end{array}$ & $\begin{array}{l}\text { Boulay et al, } \\
\qquad 2006^{20}\end{array}$ & $\begin{array}{l}\text { Roussouly } \\
2006^{24}\end{array}$ \\
\hline No. subjects & 75 & 160 & 300 & 49 & 149 & 153 \\
\hline Age & $49.3 \mathrm{yr}(18-80)$ & $25.7 \pm 5.5$ yr $(20-70)$ & 35 yr $(20-70)$ & $24.0 \pm 5.8$ yг $(19-50)$ & $30.8 \pm 6.0 \mathrm{yr}(19-50)$ & 27 yr (18-48) \\
\hline M:F ratio & 0.56 & 0.95 & 0.63 & 0.56 & 0.52 & 0.52 \\
\hline SVA & $-20 \pm 30$ & - & - & - & - & $35.2 \pm 19.4(-18.1-80.8)$ \\
\hline T1-SPI & - & - & $-1.4 \pm 2.7(-9.2-7.1)$ & - & - & - \\
\hline TK (T4-T12) & $41 \pm 12$ & $47.5 \pm 4.8(22.5-70.3)$ & $40.6 \pm 10.0(0-69)$ & $-43.0 \pm 13.0$ & $53.8 \pm 10.1$ (33.2-83.5) & $46.3 \pm 9.5(23.0-65.9)$ \\
\hline LL (L1-S1) & $60 \pm 12$ & $42.7 \pm 5.4(16-71.9)$ & $60.2 \pm 10.3(30-89)$ & $-60.0 \pm 10.0$ & $66.4 \pm 9.5(44.8-87.2)$ & $61.2 \pm 9.4(39.9-83.7)$ \\
\hline PI & $52 \pm 10$ & $51 \pm 5.3(33.7-83.7)$ & $54.7 \pm 10.6(33-82)$ & $-52.0 \pm 10.0$ & $53.1 \pm 9.0(33.7-77.5)$ & $50.6 \pm 10.2(27.9-82.8)$ \\
\hline PT & $15 \pm 7$ & $12.1 \pm 3.2(-5.1-30.5)$ & $13.2 \pm 6.1(-4.5-27)$ & $-11.0 \pm 5.5$ & $12.0 \pm 6.4(-2-30)$ & $11.1 \pm 5.9(-2.8-23.7)$ \\
\hline sS & $30 \pm 9$ & $39.7 \pm 4.1(21.2-65.9)$ & $41.2 \pm 8.4(17-63)$ & $-40.0 \pm 8.5$ & $41.2 \pm 7.0(0.6-19.7)$ & $39.6 \pm 7.6(17.5-63.4)$ \\
\hline
\end{tabular}

SVA indicates sagittal vertical axis; T1-SPI, T1-Spinopelvic inclination; TK, thoracic kyphosis; LL, lumbar lordosis; PI, pelvic incidence; PT, pelvic tilt; SS, sacral slope.

\section{Figure C-1: Radiographic Spino-pelvic Values}

Source: Table 1 reprinted with kind permission from Schwab, F., et al., Adult spinal deformity-postoperative standing imbalance: how much can you tolerate? An overview of key parameters in assessing alignment and planning corrective surgery. Spine (Phila Pa 1976), 2010. 35(25): p. 2224-31.

Promotional and commercial use of the material in print, digital or mobile device format is prohibited without the permission from the publisher Lippincott Williams \& Wilkins. Please contact journalpermissions@lww.com for further information 


\section{APPENDIX D. SUMMARY OF PRELIMINARY INVESTIGATIONS}

Given the multitude of ways to alter spinal curvature, including orthoses which and weights, it was important to determine which induced the most change without obstructing the thoracic and lumbar spine. To determine this, several preliminary tests were conducted to determine which method would be used in the investigation.

The aim of this study was to alter lumbar lordosis, and to evaluate the response of the pelvis during activities of daily living. To do this, a lumbar altering device which would not perch on the pelvis and would allow access to the spinal prominences was needed. For this reason, 6 orthoses configurations as well as anterior and posterior loads of $2.5 \mathrm{lbf}, 5 \mathrm{lbf}, 10 \mathrm{lbf}$, and $15 \mathrm{lbf}$ were evaluated. Two custom orthoses as well as modifications to a Clavicle Strap were made with the assistance of Rachel Sidle at Spears Prosthetics \& Orthotics/ Rehab Services. The three traditional orthoses: C.A.S.H., Jewett, and Clavicle Strap were chosen because they allowed access to the spinal prominences, where retroreflective markers would be placed, and did not require pelvic contact. The C.A.S.H and Jewett braces were positioned such that the most inferior points rested on the stomach instead of the pubis.

All orthoses were evaluated in the motion analysis lab at UTHSC, consisting of a ten camera video-based opto-electronic system (Qualisys AB, Gothenburg, Sweden). The subject was first equipped with retroreflective markers placed over the spinal processes $[38,39]$ with the addition of markers placed over the ASISs and PSISs. With and without the lordosis altering device, the subject was recorded at least twice in each condition for two seconds standing relaxed, with arms bent at the elbows, in the calibrated capture volume. This provided information on how the subject would compensate in standing. The subject was then evaluated as they walked barefooted at a self-selected speed over a minimum of five passes through the capture volume. All motion data was collected at $100 \mathrm{~Hz}$, exported to Visual 3D where it was interpolated over a maximum of 10 frames, and low-pass filtered at $7 \mathrm{~Hz}$ using a first-order Butterworth digital filter.

The thoracic, lordotic, and pelvic tilt angles were projected onto the sagittal plane, as defined by the midpoints of the ASISs and PSISs. Static trials were evaluated over the full two seconds. If a subject's spinal position changed by more than 2 deg during a trial, the trail was discarded. All walking trials for a given condition were normalized to a single gait cycle, and resampled at 101 points. An arithmetic mean was then calculated at each point, representing the average angle during a walking cycle. 
Table D-1: $\quad$ Summary of Changes of Lordosis and Anterior Pelvic Tilt from Normal

\begin{tabular}{lcc}
\hline (N) Orthoses Tested & Lumbar Angle (Mean \pm SD) & Pelvic Tilt (Mean \pm SD) \\
\hline (3) Clavicle strap & $-3 \pm 2 \mathrm{deg}$ & $2 \pm 0 \mathrm{deg}$ \\
(1) Reverse Clavicle strap & $3 \pm 1 \mathrm{deg}$ & $0 \pm 1 \mathrm{deg}$ \\
(1) C.A.S.H. & $5 \pm 2 \mathrm{deg}$ & $5 \pm 1 \mathrm{deg}$ \\
(1) Jewett & $\mathrm{N} / \mathrm{A}$ & $7 \pm 1 \mathrm{deg}$ \\
(2) Neck Pull Down & $3 \pm 4 \mathrm{deg}$ & $0 \pm 2 \mathrm{deg}$ \\
(1) Clavicle Pull Back & $-2 \pm 3 \mathrm{deg}$ & $-1.5 \pm 0 \mathrm{deg}$ \\
(1) 2.5 lb posterior weight & $1 \pm 1 \mathrm{deg}$ & $0 \pm 0 \mathrm{deg}$ \\
(1) 5 lb posterior weight & $-3 \pm 0 \mathrm{deg}$ & $0.5 \pm 0 \mathrm{deg}$ \\
(1) $10 \mathrm{lb}$ posterior weight & $-1.5 \pm 0 \mathrm{deg}$ & $2 \pm 0 \mathrm{deg}$ \\
(1) $15 \mathrm{lb}$ posterior weight & $1 \pm 0 \mathrm{deg}$ & $0 \pm 0 \mathrm{deg}$ \\
(1) $2.5 \mathrm{lb}$ anterior weight & $1 \pm 0 \mathrm{deg}$ & $-2 \pm 0 \mathrm{deg}$ \\
(1) $5 \mathrm{lb}$ anterior weight & $1 \pm 0 \mathrm{deg}$ & $-1 \pm 0 \mathrm{deg}$ \\
(1) $10 \mathrm{lb}$ anterior weight & $3.5 \pm 3 \mathrm{deg}$ & $1 \pm 0 \mathrm{deg}$ \\
(1) $15 \mathrm{lb}$ anterior weight & $-3 \pm 0.5 \mathrm{deg}$ & $1 \pm 0 \mathrm{deg}$ \\
\hline
\end{tabular}

Notes:

Positive change denotes increase in pelvic tilt or lordosis.

N/A : lumbar angle could not be measured due to obstructed marker(s) 


\section{APPENDIX E. INSTITUTIONAL REVIEW BOARD APPROVAL}

\begin{tabular}{lr}
\hline 4THE UNIVERSITY OF TENNESSEE \\
Health Science Center \\
\hline \\
Institutional Review Board \\
910 Madison Avenue, Suite 600 \\
Memphis, TN 38163 \\
Tel: (901) 448-4824
\end{tabular}

October 24, 2013

Bill Mihalko, $\mathrm{MD}, \mathrm{PhD}$

UTHSC - COM - Orthopaedic Surgery

E226 Coleman College of Medicine Building

956 Court Avenue

Memphis, TN 38163-0000

Re: 13-02677-FB

Study Title: Determination of functional relationship between lumbar spine and pelvic plane alignment

Dear Dr. Mihalko:

The IRB has received your written acceptance of and/or responses dated 10/14/0213 and $10 / 23 / 2013$ to the provisos outlined in our correspondence of $09 / 04 / 2013$ and $10 / 21 / 2013$ concerning the application for the above referenced project. The IRB has reviewed these materials and determined that they comply with proper consideration for the rights and welfare of human subjects and the regulatory requirements for the protection of human subjects. Therefore, this letter constitutes full approval by the IRB of your application Version 1.2 and the accompanying:

- Recruitment Flyer, dated 10/4/2013,

- Telephone and Email Script, dated 10/4/2013,

- Consent form, dated 10/23/2013 (stamped IRB approved 10/23/2013).

Approval of this study will be valid from 10/23/2013 to 09/4/2014 .

The IRB has determined that the informed consent form, incorporating the authorization of subjects to use their protected health information in research, complies with the federal privacy regulations as specified in 45 CFR 160 and 45 CFR 164 . In addition, in accord with 45 CFR 46.116(d), informed consent may be altered for your telephone and email screening, with the cover statement used in lieu of an informed consent interview. The requirement to secure a signed consent form is waived under 45 CFR 46.117 (c)(2). Willingness of the subject to participate will constitute adequate documentation of consent. 
In addition, the request for waiver of HIPAA authorization for the recruitment of subjects is approved.

In the event that subjects are to be recruited using solicitation materials, such as brochures, posters, web-based advertisements, etc., these materials must receive prior approval of the IRB. Any revisions in the approved application must also be submitted to and approved by the IRB prior to implementation. In addition, you are responsible for reporting any unanticipated serious adverse events or other problems involving risks to subjects or others in the manner required by the local IRB policy.

Finally, re-approval of your project is required by the IRB in accord with the conditions specified above. You may not continue the research study beyond the time or other limits specified unless you obtain prior written approval of the IRB.

Sincerely,

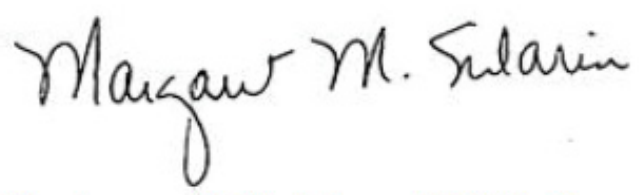

Signature applied by Margaret M Sularin on 10/24/2013 09:08:30 AM CDT

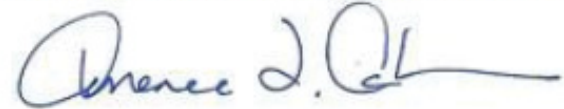

Signature applied by Terrence F Ackerman on 10/24/2013 09:09:43 AM CDT

Margaret M. Sularin, LMSW, RD, LDN, CCRP

Regulatory Specialist

UTHSC IRB
Terrence F. Ackerman, Ph.D.

Chairman

UTHSC IRB 


\section{VITA}

Casey Tyler Hebert was born in 1990.

He studied Mechanical Engineering at Gonzaga University, Spokane, Washington, from 2008 to 2012. During that time he and his engineering design team designed equipment to be implemented in to Goodrich Corporation's textile department and conducted metallurgical research on hydrogen embrittlement of ferrous alloys. His research as well his fellow peers' would later be published in "Advances in Materials Science for Environmental and Energy Technologies II". In 2012, he graduated with a 3.4 GPA and acceptance into the University of Tennessee Health Science Center's Masters of Science in Biomedical Engineering and Imaging program.

At the University of Tennessee Health Science Center, under the guidance of Dr. William Mihalko M.D., Ph.D, Casey pursued his both his studies and research with the indefatigable nature he acquired at Gonzaga. As a result Casey and his colleague Erik L. Woodard investigated over seven research projects, as well as this thesis, and showcased many of these in oral and poster presentations. Casey graduated in 2014 with a Masters of Science in Biomedical Engineering and Imaging, focusing in biomechanics with a 3.95 GPA. 